\title{
Automorphisms of Ordinary Differential Equations
}

\author{
Václav Tryhuk and Veronika Chrastinová \\ Department of Mathematics, Faculty of Civil Engineering, Brno University of Technology, Veveři 331/95, 602 00 Brno, Czech Republic
}

Correspondence should be addressed to Václav Tryhuk; tryhuk.v@fce.vutbr.cz

Received 26 July 2013; Accepted 4 October 2013; Published 28 January 2014

Academic Editor: Josef Diblik

Copyright (C) 2014 V. Tryhuk and V. Chrastinová. This is an open access article distributed under the Creative Commons Attribution License, which permits unrestricted use, distribution, and reproduction in any medium, provided the original work is properly cited.

\begin{abstract}
The paper deals with the local theory of internal symmetries of underdetermined systems of ordinary differential equations in full generality. The symmetries need not preserve the choice of the independent variable, the hierarchy of dependent variables, and the order of derivatives. Internal approach to the symmetries of one-dimensional constrained variational integrals is moreover proposed without the use of multipliers.
\end{abstract}

\section{Preface}

The theory of symmetries of determined systems (the solution depends on constants) of ordinary differential equations was ultimately established in Lie's and Cartan's era in the most possible generality and the technical tools (infinitesimal transformations and moving frames) are well known. Recall that the calculations are performed in finite-dimensional spaces given in advance and the results are expressed in terms of Lie groups or Lie-Cartan pseudogroups.

We deal with underdetermined systems (more unknown functions than the number of equations) of ordinary differential equations here. Then the symmetry problem is rather involved. Even the system of three first-order quasilinear equations with four unknown functions (equivalently, three Pfaffian equations with five variables) treated in the famous Cartan's article [1] and repeatedly referred to in actual literature was not yet clearly explained in detail. Paradoxically, the common tools (the calculations in given finite-order jet space) are quite sufficient for this particular example. We will later see that they are insufficient to analyze the seemingly easier symmetry problem of one first-order equation with three unknown functions (alternatively, two Pfaffian equations with five variables) in full generality since the order of derivatives need not be preserved in this case and the finite-order jet spaces may be destroyed. Recall that even the higher-order symmetries (automorphisms) of empty systems of differential equations (i.e., of the infinite order jet spaces without any additional differential constraints) are nontrivial [2-4] and cannot be included into the classical LieCartan theory of transformation groups. Such symmetries need not preserve any finite-dimensional space and therefore the invariant differential forms (the Maurer-Cartan forms, the moving coframes) need not exist.

Let us outline the very core of the subject for better clarity by using the common jet terminology. We start with the higher-order transformations of curves $w^{i}=w^{i}(x)$ $(i=1, \ldots, m)$ lying in the space $\mathbb{R}^{m+1}$ with coordinates $x, w^{1}, \ldots, w^{m}$. The transformations are defined by certain formulae

$$
\begin{gathered}
\bar{x}=W\left(x, \ldots, w_{s}^{j}, \ldots\right), \\
\bar{w}^{i}=W^{i}\left(x, \ldots, w_{s}^{j}, \ldots\right) \quad(i=1, \ldots, m),
\end{gathered}
$$

where the $C^{\infty}$-smooth real-valued functions $W, W^{i}$ depend on a finite number of the familiar jet variables

$$
w_{s}^{j}=\frac{d^{s} w^{j}}{d x^{s}} \quad(j=1, \ldots, m ; s=0,1, \ldots) .
$$

The resulting curve $\bar{w}^{i}=\bar{w}^{i}(\bar{x})(i=1, \ldots, m)$ again lying in $\mathbb{R}^{m+1}$ appears as follows. We put

$$
\bar{x}=W\left(x, \ldots, \frac{d^{s} w^{j}(x)}{d x^{s}}, \ldots\right)=w(x)
$$


and assuming

$$
\begin{array}{r}
w^{\prime}(x)=(D W)\left(x, \ldots, \frac{d^{s} w^{j}(x)}{d x^{s}}, \ldots\right) \neq 0 \\
\left(D=\frac{\partial}{\partial x}+\sum w_{s+1}^{j} \frac{\partial}{\partial w_{s}^{j}}\right),
\end{array}
$$

there exists the inverse function $x=w^{-1}(\bar{x})$ which provides the desired result

$$
\bar{w}^{i}(\bar{x})=W^{i}\left(w^{-1}(\bar{x}), \ldots, \frac{d^{s} w^{j}}{d x^{s}}\left(w^{-1}(\bar{x})\right), \ldots\right) .
$$

One can also easily obtain the well-known prolongation formula

$$
\begin{aligned}
& \bar{w}_{s}^{i}=W_{s}^{i}\left(x, \ldots, w_{s}^{j}, \ldots\right) \\
& \left(W_{s+1}^{i}=\frac{D W_{s}^{i}}{D W} ; i=1, \ldots, m ; s=0,1, \ldots ; W_{0}^{i}=W^{i}\right)
\end{aligned}
$$

for the derivatives $\bar{w}_{r}^{i}=d^{r} \bar{w}^{i} / d \bar{x}^{r}$ by using the Pfaffian equations

$$
d \bar{w}_{r}^{i}-\bar{w}_{r+1}^{i} d \bar{x}=0 \quad(i=1, \ldots, m ; r=0,1, \ldots) .
$$

Functions $W$ satisfying (4) and $W^{i}$ may be arbitrary here.

At this place, in order to obtain coherent theory, introduction of the familiar infinite-order jet space of $x$-parametrized curves briefly designated as $\mathbf{M}(m)$ with coordinates $x, w_{r}^{i}(i=$ $1, \ldots, m ; r=0,1, \ldots)$ is necessary. Then formulae $((1),(6))$ determine a mapping $\mathbf{m}: \mathbf{M}(m) \rightarrow \mathbf{M}(m)$, a morphism of the jet space $\mathbf{M}(m)$. If the inverse $\mathbf{m}^{-1}$ given by certain formulae

$$
\begin{gathered}
x=\bar{W}\left(\bar{x}, \ldots, \bar{w}_{s}^{j}, \ldots\right), \\
w_{r}^{i}=\bar{W}_{r}^{i}\left(\bar{x}, \ldots, \bar{w}_{s}^{j}, \ldots\right) \quad(i=1, \ldots, m ; r=0,1, \ldots)
\end{gathered}
$$

exists, we speak of an automorphism (in alternative common terms, symmetry) $\mathbf{m}$ of the jet space $\mathbf{M}(m)$. It should be noted that we tacitly deal with the local theory in the sense that all formulae and identities, all mappings, and transformation groups to follow are in fact considered only on certain open subsets of the relevant underlying spaces which is not formally declared by the notation. Expressively saying, in order to avoid the clumsy purism, we follow the reasonable 19 th century practice and do not rigorously indicate the true definition domains.

After this preparation, a system of differential equations is traditionally identified with the subspace $\mathbf{M} \subset \mathbf{M}(m)$ given by certain equations

$$
\begin{aligned}
& D^{r} G^{k}=0 \\
& \left(k=1, \ldots K ; r=0,1, \ldots ; G^{k}=G^{k}\left(x, \ldots, w_{s}^{j}, \ldots\right)\right) .
\end{aligned}
$$

(We tacitly suppose that $\mathbf{M} \subset \mathbf{M}(m)$ is a "reasonable subspace" and omit the technical details.) This is the infinitely prolonged system. The total derivative vector field $D$ defined on $\mathbf{M}(m)$ is tangent to the subspace $\mathbf{M} \subset \mathbf{M}(m)$ and may be regarded as a vector field on $\mathbf{M}$, as well. The morphism $\mathbf{m}: \mathbf{M}(m) \rightarrow \mathbf{M}(m)$ transforms $\mathbf{M} \subset \mathbf{M}(m)$ into the subspace $\mathbf{m} \mathbf{M} \subset \mathbf{m} \mathbf{M}(m) \subset \mathbf{M}(m)$ given by the equations

$$
\begin{gathered}
\left(D^{r} G^{k}\right)\left(W, \ldots, W_{s}^{j}, \ldots\right)=0 \\
(k=1, \ldots, K ; r=0,1, \ldots) .
\end{gathered}
$$

This is again a system of differential equations. In our paper, we are interested only in the particular case when $\mathbf{m M}=\mathbf{M}$. Then, if the inverse $\mathbf{~ m}^{-1}$ locally exists on a neighbourhood of the subspace $\mathbf{M} \subset \mathbf{M}(m)$ in the total jet space, we speak of the external symmetry $\mathbf{m}$ of the system of differential equations (9). Let us, however, deal with the natural restriction $\overline{\mathbf{m}}$ : $\mathbf{M} \rightarrow \mathbf{M}$ of the mapping $\mathbf{m}$ to the subspace $\mathbf{M}$. If there exists the inverse $\overline{\mathbf{m}}^{-1}: \mathbf{M} \rightarrow \mathbf{M}$ of the restriction, we speak of the internal symmetry. Internal symmetries do not depend on the localizations of $\mathbf{M}$ in $\mathbf{M}(m)$. More precisely, differential equations can be introduced without any reference to jet spaces and the internal symmetries can be defined without the use of localizations. On this occasion, we are also interested in groups of internal symmetries. They are generated by special vector fields, the infinitesimal symmetries.

In the actual literature, differential equations are as a rule considered in finite-dimensional jet spaces. Then the internal and external symmetries become rather delicate and differ from our concepts since the higher-order symmetries are not taken into account. We will not discuss such conceptual confusion in this paper with the belief that the following two remarks (and Remark 5) should be quite sufficient in this respect.

Remark 1 (on the symmetries). The true structure of the jet space $\mathbf{M}(m)$ is determined by the contact module $\Omega(m)$ which involves all contact forms

$$
\begin{aligned}
& \omega=\sum a_{r}^{i} \omega_{r}^{i} \\
& \left(\omega_{r}^{i}=d w_{r}^{i}-w_{r+1}^{i} d x, \text { finite sum, arbitrary coefficients }\right) .
\end{aligned}
$$

Then the above morphisms $\mathbf{m}: \mathbf{M}(m) \rightarrow \mathbf{M}(m)$ given in $((1),(6))$ are characterized by the property $\mathbf{m}^{*} \Omega(m) \subset \Omega(m)$. Recall that invertible morphisms are automorphisms. Let us introduce the subspace $\mathbf{i}: \mathbf{M} \subset \mathbf{M}(m)$ of all points (9). This $\mathbf{M}$ is equipped with the restriction $\Omega=\mathbf{i}^{*} \Omega(m)$ of the contact module. Recall that we are interested only in the case $\mathbf{m} \mathbf{M}=\mathbf{M}$ (abbreviation of $\mathbf{m i M}=\mathbf{i} \mathbf{M}$ ). Let $\overline{\mathbf{m}}: \mathbf{M} \rightarrow$ $\mathbf{M}$ be the restriction of $\mathbf{m}$. If $\mathbf{m}$ is a morphism then $\overline{\mathbf{m}}$ is a morphism in the sense that $\overline{\mathbf{m}}^{*} \Omega \subset \Omega$. Recall that we have the internal symmetry, if $\overline{\mathbf{m}}$ is moreover invertible. If also $\mathbf{m}$ is invertible, we have the external symmetry $\overline{\mathbf{m}}$. The internal symmetries can be defined without any reference to $\mathbf{m}$ and $\mathbf{M}(m)$ as follows. Let $\overline{\mathbf{m}}: \mathbf{M} \rightarrow \mathbf{M}$ be any invertible mapping such that $\overline{\mathbf{m}}^{*} \Omega \subset \Omega$. This $\overline{\mathbf{m}}$ can be always extended to a morphism $\mathbf{m}: \mathbf{M}(m) \rightarrow \mathbf{M}(m)$ of the ambient jet space. 
(Hint, recurrence (6) holds true both in $\mathbf{M}(m)$ and in $\mathbf{M}$.) So we may conclude that such $\overline{\mathbf{m}}$ is just the internal symmetry. Moreover, if there exists invertible extension $\mathbf{m}$ of $\overline{\mathbf{m}}$, then $\overline{\mathbf{m}}$ is even the external symmetry but the latter concept already depends on the localization $\mathbf{i}$ of $\mathbf{M}$ in $\mathbf{M}(m)$.

Remark 2 (on infinitesimal symmetries). Let us consider a vector field

$$
Z=z \frac{\partial}{\partial x}+\sum z_{r}^{i} \frac{\partial}{\partial w_{r}^{i}}
$$

(infinite sum, arbitrary coefficients)

on the jet space $\mathbf{M}(m)$. Let us moreover suppose $\mathscr{L}_{Z} \Omega(m) c$ $\Omega(m)$ from now on (where $\mathscr{L}_{Z}$ denotes the Lie derivative see also Definition 8). In common terminology, such vector fields $Z$ are called generalized (higher-order, Lie-Bäcklund) infinitesimal symmetries of the jet space $\mathbf{M}(m)$. However $Z$ need not in general generate any true group of transformations and we therefore prefer the "unorthodox" term a variation $Z$ here. (See Section 7 and especially Remark 35 where the reasons for this term are clarified.) The common term infinitesimal symmetry is retained only for the favourable case when $Z$ generates a local one-parameter Lie group [5]. Let us consider the above subspace $\mathbf{i}: \mathbf{M} \subset \mathbf{M}(m)$. If $Z$ is tangent to $\mathbf{M}$, then there exists the natural restriction $\bar{Z}$ of $Z$ to $\mathbf{M}$. Clearly $\mathscr{L}_{\bar{Z}} \Omega \subset \Omega$ and we speak of the (internal) variation $\bar{Z}$. If $\bar{Z}$ moreover generates a group in $\mathbf{M}$, we have the (internal) infinitesimal symmetry $\bar{Z}$. The internal concepts on $\mathbf{M}$ can be easily introduced without any reference to the ambient space $\mathbf{M}(m)$. This is not the case for the concept of the external infinitesimal symmetry $\bar{Z}$ which supposes that appropriate extension $Z$ of $\bar{Z}$ on the ambient space $\mathbf{M}(m)$ generates a Lie group.

We deal only with the internal symmetries and infinitesimal symmetries in this paper. It is to be noted once more that infinite-dimensional underlying spaces are necessary if we wish to obtain a coherent theory. The common technical tools invented in the finite-dimensional spaces will be only slightly adapted; alas, the ingenious methods proposed, for example, in [6-8] seem to be not suitable for this aim and so we undertake the elementary approach [9] here.

\section{Technical Tools}

We introduce infinite-dimensional manifold $\mathbf{M}$ modelled on the space $\mathbb{R}^{\infty}$ with local coordinates $h^{1}, h^{2}, \ldots$ in full accordance with [9]. The manifold $\mathbf{M}$ is equipped with the structural algebra $\mathscr{F}(\mathbf{M})$ of $C^{\infty}$-smooth functions expressed as $f=f\left(h^{1}, \ldots, h^{m(f)}\right)$ in terms of coordinates. Transformations (mappings) $\mathbf{m}: \mathbf{M} \rightarrow \mathbf{M}$ are (locally) given by certain formulae

$$
\mathbf{m}^{*} h^{i}=H^{i}\left(h^{1}, \ldots, h^{m(i)}\right) \quad\left(H^{i} \in \mathscr{F}(\mathbf{M}) ; i=1,2, \ldots\right),
$$

and analogous (invertible) formulae describe the change of coordinates at the overlapping coordinate systems.
Let $\Phi(\mathbf{M})$ be the $\mathscr{F}(\mathbf{M})$-module of differential 1-forms

$$
\varphi=\sum f^{i} d g^{i} \quad\left(f^{i}, g^{i} \in \mathscr{F}(\mathbf{M}) \text {; finite sum }\right) .
$$

The familiar rules of exterior calculus can be applied without any change, in particular $\mathbf{m}^{*} \varphi=\sum \mathbf{m}^{*} f^{i} d \mathbf{m}^{*} g^{i}$ for the above transformation $\mathbf{m}$.

Let $\mathscr{T}(\mathbf{M})$ be the $\mathscr{F}(\mathbf{M})$-module of vector fields $Z$. In terms of coordinates we have

$$
\begin{aligned}
& Z=\sum z^{i} \frac{\partial}{\partial h^{i}} \\
& \left(z^{i}=z^{i}\left(h^{1}, \ldots, h^{m(i)}\right) \in \mathscr{F}(\mathbf{M}), \text { infinite sum }\right),
\end{aligned}
$$

where the coefficients $z^{i}$ may be quite arbitrary. We identify $Z$ with the linear functional on $\Phi(\mathbf{M})$ determined by the familiar duality pairing

$$
\left.d h^{i}(Z)=Z\right\rfloor d h^{i}=Z h^{i}=z^{i} \quad(i=1,2, \ldots) .
$$

With this principle in mind, if certain forms $\varphi^{1}, \varphi^{2}, \ldots \epsilon$ $\Phi(\mathbf{M})$ generate the $\mathscr{F}(\mathbf{M})$-module, then the values

$$
\left.\varphi^{i}(Z)=Z\right\rfloor \varphi^{i}=\bar{z}^{i} \in \mathscr{F}(\mathbf{M}) \quad(i=1,2, \ldots)
$$

uniquely determine the vector field $Z$ and (17) can be very expressively (and unorthodoxly) recorded by

$$
Z=\sum \bar{z}^{i} \frac{\partial}{\partial \varphi^{i}} \quad\left(\bar{z}^{i}=\varphi^{i}(Z) \in \mathscr{F}(\mathbf{M}), \text { infinite sum }\right) .
$$

This is a mere symbolical record, not the true infinite series. However, if $\varphi^{1}, \varphi^{2}, \ldots$ is a basis of the module $\Phi(\mathbf{M})$ in the sense that every $\varphi \in \Phi(\mathbf{M})$ admits a unique representation $\varphi=\sum f^{i} \varphi^{i}\left(f^{i} \in \mathscr{F}(\mathbf{M})\right.$, finite sum) then the coefficients $\bar{z}_{i}$ can be quite arbitrary and (18) may be regarded as a true infinite series. The arising vector fields $\partial / \partial \varphi^{1}, \partial / \partial \varphi^{2}, \ldots$ provide a weak basis (infinite expansions, see [9]) of $\mathscr{T}(\mathbf{M})$ dual to the basis $\varphi^{1}, \varphi^{1}, \ldots$ of $\Phi(\mathbf{M})$. In this transcription, (15) is alternatively expressed as

$$
Z=\sum z^{i} \frac{\partial}{\partial d h^{i}} \quad\left(z^{i} \in \mathscr{F}(\mathbf{M}), \text { infinite sum }\right) .
$$

We recall the Lie derivative $\left.\left.\mathscr{L}_{Z}=Z\right\rfloor d+d Z\right\rfloor$ acting on exterior differential forms. The image $\mathbf{m}_{*} Z$ of a vector field defined by the property

$$
\mathbf{m}^{*}\left(\mathbf{m}_{*} Z\right) f=Z \mathbf{m}^{*} f \quad(f \in \mathscr{F}(\mathbf{M}))
$$

need not exist. It is defined if $\mathbf{m}$ is invertible.

We consider various submodules $\Omega \subset \Phi(\mathbf{M})$ of differential forms together with the relevant orthogonal submodules $\Omega^{\perp} \subset \mathscr{T}(\mathbf{M})$ consisting of all vector fields $Z \in \mathscr{T}(\mathbf{M})$ such that $\omega(Z)=0(\omega \in \Omega)$. The existence of (local) $\mathscr{F}(\mathbf{M})$ bases in all submodules of $\Phi(\mathbf{M})$ to appear in our reasonings is tacitly postulated. Dimension of an $\mathscr{F}(\mathbf{M})$-module is the number of elements of an $\mathscr{F}(\mathbf{M})$-basis. Omitting some "exceptional points," it may be confused with the dimension 
of the corresponding $\mathbb{R}$-module (the localization) at a fixed place $\mathbf{P} \in \mathbf{M}$. On this occasion, it should be noted that the image

$$
\mathbf{m}_{*} Z_{\mathbf{P}} \quad\left(\left(\mathbf{m}_{*} Z_{\mathbf{P}}\right)_{\mathbf{Q}} f=Z_{\mathbf{P}}\left(\mathbf{m}^{*} f\right), \mathbf{m} \mathbf{P}=\mathbf{Q}, \mathbf{P} \in \mathbf{M}\right)
$$

of a tangent vector $Z_{\mathbf{P}}$ at $\mathbf{P}$ exists as a vector at the place $\mathbf{Q}$.

Let us also remark with regret that any rigorous exposition of classical analysis in the infinite-dimensional space $\mathbb{R}^{\infty}$ is not yet available; however, certain adjustments of finite-dimensional results are not difficult. For instance, the following invertibility theorem will latently occur in the proof of Theorem 20 .

Theorem 3. A mapping $\mathbf{m}: \mathbf{M} \rightarrow \mathbf{M}$ is invertible if and only if any of the following equivalent conditions is satisfied: the pull-back $\mathbf{m}^{*}: \mathscr{F}(\mathbf{M}) \rightarrow \mathscr{F}(\mathbf{M})$ is invertible, the pullback $\mathbf{m}^{*}: \Phi(\mathbf{M}) \rightarrow \Phi(\mathbf{M})$ is invertible, and if $\varphi^{1}, \varphi^{2}, \ldots$ is a (fixed, equivalently: arbitrary) basis of module $\Phi(\mathbf{M})$, then $\mathbf{m}^{*} \varphi^{1}, \mathbf{m}^{*} \varphi^{2}, \ldots$ again is a basis.

Hint. A nonlinear version of the familiar Gauss elimination procedure for infinite dimension [9] provides a direct proof with difficulties concerning the definition domain of the resulting inverse mapping. Nevertheless if $\mathbf{m}$ is moreover a morphism of a diffiety (see Definition 8 ) then the prolongation procedure ensures the local existence of $\mathbf{m}^{-1}$ in the common sense.

\section{Fundamental Concepts}

We introduce a somewhat unusual intrinsical approach to underdetermined systems of ordinary differential equations in terms of the above underlying space $\mathbf{M}$, a submodule $\Omega \subset$ $\Phi(\mathbf{M})$ of differential 1-forms, and its orthogonal submodule $\mathscr{H}=\Omega^{\perp} \subset \mathscr{T}(\mathbf{M})$ of vector fields.

Definition 4. A codimension one submodule $\Omega \subset \Phi(\mathbf{M})$ is called a diffiety if there exists a good filtration

$$
\Omega_{*}: \Omega_{0} \subset \Omega_{1} \subset \cdots \subset \Omega=\cup \Omega_{l}
$$

by finite-dimensional submodules $\Omega_{l} \subset \Omega(l=0,1, \ldots)$ such that

$$
\begin{gathered}
\mathscr{L}_{\mathscr{H}} \Omega_{l} \subset \Omega_{l+1} \quad(\text { all } l), \\
\mathscr{L}_{\mathscr{H}} \Omega_{l}+\Omega_{l}=\Omega_{l+1} \quad(l \text { large enough }) .
\end{gathered}
$$

To every subset $\Theta \subset \Phi(\mathbf{M})$, let $\mathscr{L}_{\mathscr{H}} \Theta \subset \Phi(\mathbf{M})$ denote the submodule with generators $\mathscr{L}_{Z} \vartheta(Z \in \mathscr{H}, \vartheta \in \Theta)$. Since $\Theta \subset \mathscr{L}_{\mathscr{H}} \Theta$ (easy), the second requirement (23) can be a little formally simplified as $\mathscr{L}_{\mathscr{H}} \Omega_{l}=\Omega_{l+1}$.

Remark 5. This is a global coordinate-free definition; however, we again deal only with the local theory from now on in the sense that the definition domains (of filtrations (22), of independent variable $x$ to follow, and so on) are not specified. It should be noted on this occasion that the common geometrical approach [6-8] to differential equations rests on the use of the rigid structure of finite-order jets. Many classical concepts then become incorrect, if the higher-order mappings are allowed but we cannot adequately discuss this important topic here. Rather subtle difficulties are also passed over already in the common approach to the fundamental jet theory. For instance, smooth curves in the plane $\mathbb{R}^{2}$ with coordinates $x, y$ are parametrized either by $x$ (i.e., $y=y(x)$ ) or by coordinate $y$ (i.e., $x=x(y)$ ) in the common so-called "geometrical" approach [6-8]. However, then already the Lie's classical achievements concerning contact transformations $[10,11]$ with curves parametrized either by $p=d y / d x$ or by $q=d x / d y$ cannot be involved. Quite analogously, the "higher-order" parameterizations and mappings [2-5] are in fact rejected in the common "rigid" jet theory with a mere point symmetries.

Definition 6. Let a differential $d x(x \in \mathscr{F}(\mathbf{M}))$ generate together with $\Omega$ the total module $\Phi(\mathbf{M})$ of all differential 1forms. Then $x$ is called the independent variable to diffiety $\Omega$. The vector field $D=D_{x}$ (abbreviation) such that

$$
\begin{aligned}
& D \in \mathscr{H}, \quad D x=d x(D)=1 \\
& \left(\text { symbolically } D=\frac{\partial}{\partial d x}+\sum_{\omega \in \Omega} 0 \cdot \frac{\partial}{\partial \omega}\right)
\end{aligned}
$$

is called total (or formal) derivative of $\Omega$ with respect to the independent variable $x$. This vector field $D$ is a basis of the one-dimensional module $\mathscr{H}=\Omega^{\perp}$ for every fixed particular choice of the independent variable $x$.

Remark 7. Let us state some simple properties of diffieties. The proofs are quite easy and may be omitted. A form $\varphi \in$ $\Phi(\mathbf{M})$ is lying in $\Omega$ if and only if $\varphi(D)=0$. In particular $\mathscr{L}_{D} \Omega \subset \Omega$ in accordance with the identities

$$
\begin{array}{r}
\left.\left.\mathscr{L}_{D} \omega=D\right\rfloor d \omega+d \omega(D)=D\right\rfloor d \omega, \\
\left(\mathscr{L}_{D} \omega\right)(D)=d \omega(D, D)=0 \\
(\omega \in \Omega) .
\end{array}
$$

(This trivial property clarifies the more restrictive condition (23).) Moreover clearly

$$
\begin{array}{r}
D f d g-D g d f, \quad d f-D f d x \in \Omega \\
(f, g \in \mathscr{F}(\mathbf{M}))
\end{array}
$$

and in particular

$$
\begin{array}{r}
D h^{i} d h^{j}-D h^{j} d h^{i}, \quad d h^{i}-D h^{i} d x \in \Omega \\
(i, j=1,2, \ldots)
\end{array}
$$

for all coordinates. We have very useful $\mathscr{F}(\mathbf{M})$-generators of diffiety $\Omega$. The independent variable and the filtrations (22) can be capriciously modified. In particular the $c$-lift [9]

$$
\begin{array}{r}
\Omega_{*+c}=\widetilde{\Omega}_{*}: \widetilde{\Omega}_{0} \subset \widetilde{\Omega}_{1} \subset \cdots \subset \Omega=\cup \widetilde{\Omega}_{l} \\
\left(\widetilde{\Omega}_{l}=\Omega_{l+c}, c=0,1, \ldots\right)
\end{array}
$$


with $c$ large enough ensures that $\widetilde{\Omega}_{l+1}=\mathscr{L}_{\mathscr{H}} \widetilde{\Omega}_{l}+\widetilde{\Omega}_{l}$ for all $l \geq 0$. We will be, however, interested just in the reverse concept " $\Omega_{*-c}$ " latently involved in the "standard adaptation" of filtrations to appear later on.

Definition 8. A transformation $\mathbf{m}: \mathbf{M} \rightarrow \mathbf{M}$ is called a morphism of the diffiety $\Omega$ if $\mathbf{m}^{*} \Omega \subset \Omega$. Invertible morphisms are automorphisms (or symmetries) of $\Omega$. A vector field $Z \in \mathscr{T}(\mathbf{M})$ satisfying $\mathscr{L}_{Z} \Omega \subset \Omega$ is called the variation of $\Omega$. If moreover $Z$ (locally) generates a one-parameter group of transformations, we speak of the infinitesimal symmetry $Z$ of diffiety $\Omega$.

Remark 9. Let us mention the transformation groups in more detail. A local one-parameter group of transformations $\mathbf{m}(\lambda): \mathbf{M} \rightarrow \mathbf{M}$ is given by certain formulae

$$
\mathbf{m}(\lambda)^{*} h^{i}=H^{i}\left(h^{1}, \ldots, h^{m(i)} ; \lambda\right) \quad(i=1,2, \ldots ;-\varepsilon<\lambda<\varepsilon)
$$

in terms of local coordinates, where $\mathbf{m}(\lambda+\mu)=$ $\mathbf{m}(\lambda) \mathbf{m}(\mu), \mathbf{m}(0)=i d$ is supposed. Then the special vector field (15) defined by

$$
\begin{array}{r}
z^{i}=\left.\frac{\partial}{\partial \lambda} \mathbf{m}(\lambda)^{*} h^{i}\right|_{\lambda=0}=\frac{\partial}{\partial \lambda} H^{i}\left(h^{1}, \ldots, h^{m(i)} ; 0\right) \\
(i=1,2, \ldots)
\end{array}
$$

is called the infinitesimal transformation of the group (29). In the opposite direction, we recall that a general vector field (15) generates the local group (29) if and only if the Lie system

$$
\begin{array}{r}
\frac{\partial H^{i}}{\partial \lambda}=z^{i}\left(H^{1}, \ldots, H^{n(i)}\right), \quad H^{i}\left(h^{1}, \ldots, h^{m(i)} ; 0\right)=h^{i} \\
(i=1,2, \ldots)
\end{array}
$$

is satisfied. Alas, a given vector field (19) need not in general generate any transformation group since the Lie system need not admit any solution (29).

With all fundamental concepts available, let us eventually recall the familiar and thoroughly discussed in [9] interrelation between the diffieties and the corresponding classical concept of differential equations for the convenience of reader. In brief terms, the idea is quite simple. A given system of differential equations is represented by a system of Pfaffian equations $\omega=0$ and the module $\Omega$ generated by such 1 -forms $\omega$ is just the diffiety. More precisely, we deal with the infinite prolongations as follows.

In one direction, let a system of underdetermined ordinary differential equations be given. We may deal with the first-order system

$$
\begin{array}{r}
\frac{d w^{j}}{d x}=f^{j}\left(x, w^{1}, \ldots, w^{m}, \frac{d w^{J+1}}{d x}, \ldots, \frac{d w^{m}}{d x}\right) \\
(j=1, \ldots, J)
\end{array}
$$

without any true loss of generality. Then (32) completed with

$$
\frac{d w_{s}^{k}}{d x}=w_{s+1}^{k} \quad\left(w_{0}^{k}=w^{k} ; k=J+1, \ldots, m ; s=0,1, \ldots\right)
$$

provides the infinite prolongation. The corresponding diffiety $\Omega$ is generated by the forms

$$
\begin{aligned}
& d w^{j}-f^{j} d x, \quad d w_{s}^{k}-w_{s+1}^{k} d x \\
& (j=1, \ldots, J ; k=J+1, \ldots, m ; s=0,1, \ldots)
\end{aligned}
$$

in the space $\mathbf{M}$ with coordinates

$$
\begin{gathered}
w^{j} \quad(j=1, \ldots, J), \\
w_{s}^{k} \quad\left(k=J+1, \ldots, m ; s=0,1, \ldots ; w_{0}^{k}=w^{k}\right) .
\end{gathered}
$$

Clearly

$$
D_{x}=\frac{\partial}{\partial x}+\sum f^{j} \frac{\partial}{\partial w^{j}}+\sum w_{s+1}^{k} \frac{\partial}{\partial w_{s}^{k}} \in \mathscr{H}
$$

is the total derivative and the submodules $\Omega_{l} \subset \Omega$ of all forms (34) with $s \leq l$ determine a quite simple filtration (22) with respect to the order of contact forms. (Hint: use the formulae

$$
\begin{aligned}
& \mathscr{L}_{D}\left(d w^{j}-f^{j} d x\right) \\
& \quad=\sum \frac{\partial f^{j}}{\partial w^{j}}\left(d w^{j}-f^{j} d x\right)+\sum \frac{\partial f^{j}}{\partial w_{1}^{j}}\left(d w_{1}^{j}-w_{2}^{j} d x\right)
\end{aligned}
$$

and $\mathscr{L}_{D}\left(d w_{s}^{j}-w_{s+1}^{j} d x\right)=d w_{s+1}^{j}-w_{s+2}^{j} d x$. However, there exist many other and more useful filtrations; see the examples to follow later on.

The particular case $J=0$ of the empty system (32) can be easily related to the case of the jet space $\mathbf{M}(m)$ of all $x$ parametrized curves in $\mathbb{R}^{m+1}$ of the Section 1 . The relevant diffiety is identified with the module $\Omega(m)$ of all contact forms (11), of course.

In the reverse direction, let a diffiety $\Omega$ be given on the space $\mathbf{M}$. In accordance with (27), the forms $d h^{i}-D h^{i} d x$ ( $i=$ $1,2, \ldots)$ generate $\Omega$. So we have the Pfaffian system $d h^{i}-$ $D h^{i} d x=0(i=1,2, \ldots)$ and therefore the system of differential equations

$$
\frac{d h^{i}}{d x}=g^{i}\left(x, h^{1}, \ldots, h^{m(i)}\right) \quad\left(i=1,2, \ldots ; g^{i}=D h^{i}\right)
$$

of rather unpleasant kind. Then, due to the existence of a filtration (22) and (23), one can obtain also the above classical system of differential equations (32) together with the prolongation (33) by means of appropriate change of coordinates [9]. This is, however, a lengthy procedure and a shorter approach can be described as follows. Let the second requirement (23) be satisfied, if $l \geq L$. Suppose that the forms $\omega^{j}=\sum a_{i}^{j} d h^{i}\left(j=1, \ldots, J=\operatorname{dim} \Omega_{L}\right)$ generate module $\Omega_{L}$. Then all forms

$$
\mathscr{L}_{D}^{k} \omega^{j} \quad(j=1, \ldots, J ; k=0,1, \ldots)
$$


generate the diffiety $\Omega$. The corresponding Pfaffian system $\mathscr{L}_{D}^{k} \omega^{j}=0$ is equivalent to certain infinite prolongation of differential equations, namely,

$$
\begin{gathered}
\omega^{j}=\sum a_{i}^{j} d h^{i}=0 \text { is equivalent to } \sum a_{i}^{j} \frac{d h^{i}}{d x}=0, \\
\mathscr{L}_{D} \omega^{j}=\sum D a_{i}^{j} d h^{i}+\sum a_{i}^{j} d D h^{i} \\
=0 \text { is equivalent to } \frac{d}{d x} \sum a_{i}^{j} \frac{d h^{i}}{d x}=0
\end{gathered}
$$

(direct verification), and in general

$$
\begin{aligned}
\mathscr{L}_{D}^{k} \omega^{j} & =\sum D^{k} a_{i}^{j} d h^{i}+\cdots+\sum a_{i}^{j} d D^{k} h^{i} \\
& =0 \text { is equivalent to } \frac{d^{k}}{d x^{k}} \sum a_{i}^{j} \frac{d h^{i}}{d x}=0 .
\end{aligned}
$$

We have the infinite prolongation of the classical system $\sum a_{i}^{j} d h^{i} / d x=0(j=1, \ldots, J)$ and this is just the system that corresponds to diffiety $\Omega$.

Altogether taken, differential equations uniquely determine the corresponding diffieties; however, a given diffiety leads to many rather dissimilar but equivalent systems of differential equations with regard to the additional choice of dependent and independent variables.

Remark 10. Definitions 4-8 make good sense even if $\mathbf{M}$ is a finite-dimensional manifold and then provide the wellknown intrinsical approach to determined systems of differential equations. They are identified with vector fields (better, fields of directions) in the finite-dimensional space $\mathbf{M}$. Choosing a certain independent variable $x$, the equations are represented by the vector field $D_{x}$ or, more visually, by the corresponding $D_{x}$-flow. The general theory becomes trivial; we may, for example, choose $\Omega_{l}=\Omega$ for all $l$ in filtration (22).

\section{On the Structure of Diffieties}

Definition 11. To every submodule $\Theta \subset \Omega$ of a diffiety $\Omega \subset$ $\Phi(\mathbf{M})$, let $\operatorname{Ker} \Theta \subset \Theta$ be the submodule of all $\vartheta \in \Theta$ such that $\mathscr{L}_{\mathscr{H}} \vartheta \in \Theta$. Filtration (22) and (23) is called a standard one, if

$$
\begin{aligned}
& \operatorname{Ker} \Omega_{l+1}=\Omega_{l} \quad(l \geq 0), \\
& \operatorname{Ker}^{2} \Omega_{0}=\operatorname{Ker} \Omega_{0} \neq \Omega_{0} .
\end{aligned}
$$

For every $\omega \in \Omega$, the first condition ensures that the inclusions $\omega \in \Omega_{l}, \mathscr{L}_{D} \omega \in \Omega_{l+1}$ are equivalent and the second condition ensures that $\mathscr{L}_{D} \omega \in \Omega_{0}$ implies $\mathscr{L}_{D}^{2} \omega \in \Omega_{0}$.

Theorem 12. Appropriate adaptation of some lower-order terms of a given filtration (22) and (23) provides a standard filtration in a unique manner [9]. Equivalently and in more detail, there exists unique standard filtration $\bar{\Omega}_{*}: \bar{\Omega}_{0} \subset \bar{\Omega}_{1} \subset$ $\cdots \subset \Omega=\cup \bar{\Omega}_{l}$ such that $\Omega_{l}=\bar{\Omega}_{l+c}$ for appropriate $c \in \mathbb{N}$ and all l large enough. Equivalently and briefly, there exists unique

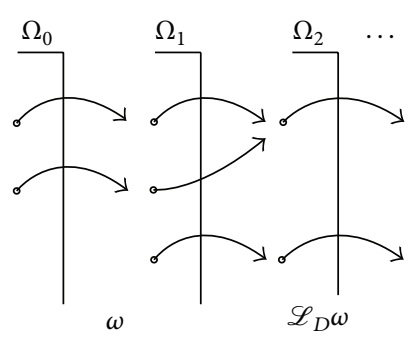

Original filtration

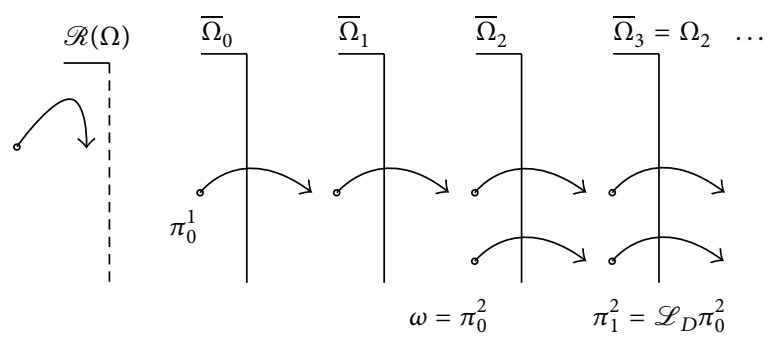

The corresponding standard filtration

FIGURE 1

standard filtration $\bar{\Omega}_{*}$ such that $\Omega_{*+c^{\prime}}=\bar{\Omega}_{*+c^{\prime \prime}}$ for appropriate $c^{\prime}, c^{\prime \prime} \in \mathbb{N}$.

Proof. The mapping $\mathscr{L}_{D}: \Omega_{l} \rightarrow \Omega_{l+1}$ naturally induces certain $\mathscr{F}(\mathbf{M})$-homomorphism

$$
D: \Omega_{l} / \Omega_{l-1} \longrightarrow \Omega_{l+1} / \Omega_{l} \quad\left(l \geq 0, \text { formally } \Omega_{-1}=0\right)
$$

of factor modules denoted by $D$ for better clarity. Homomorphisms $D$ are surjective and therefore even bijective for all $l$ large enough, say for $l \geq L$. However, the injectivity of $D$ implies $\operatorname{Ker} \Omega_{l}=\Omega_{l-1}(l \geq L)$. It follows that we have strongly decreasing sequence

$$
\begin{aligned}
\cdots & \supset \Omega_{L}\left(=\operatorname{Ker} \Omega_{L+1}\right) \supset \Omega_{L-1}\left(=\operatorname{Ker} \Omega_{L}\right) \\
& \supset \operatorname{Ker} \Omega_{L-1} \supset \operatorname{Ker}^{2} \Omega_{L-1} \supset \cdots,
\end{aligned}
$$

which necessarily terminates with the stationarity $\operatorname{Ker}^{C} \Omega_{L-1}=\operatorname{Ker}^{C+1} \Omega_{L-1}$. Denoting

$$
\begin{gathered}
\bar{\Omega}_{0}=\operatorname{Ker}^{C-1} \Omega_{L-1}, \ldots, \\
\bar{\Omega}_{C-1}=\operatorname{Ker} \Omega_{L-1}, \quad \bar{\Omega}_{C}=\Omega_{L-1}, \quad \bar{\Omega}_{C+1}=\Omega_{L}, \ldots,
\end{gathered}
$$

we have the sought strongly increasing standard filtration

$$
\begin{aligned}
& \bar{\Omega}_{*}: \bar{\Omega}_{0} \subset \bar{\Omega}_{1} \subset \cdots \subset \bar{\Omega}_{C}\left(=\Omega_{L-1}\right) \\
& \subset \bar{\Omega}_{C+1}\left(=\Omega_{L}\right) \subset \cdots \subset \Omega=\cup \bar{\Omega}_{l}
\end{aligned}
$$

of diffiety $\Omega$. In particular $\operatorname{Ker}^{2} \bar{\Omega}_{0}=\operatorname{Ker}^{C+1} \Omega_{L-1}=$ $\operatorname{Ker}{ }^{C} \Omega_{L-1}=\operatorname{Ker} \bar{\Omega}_{0}$. 
Proof of Theorem 12 was of the algorithmical nature and provides a useful standard basis of diffiety $\Omega$ as follows. Assume that the forms

$$
\tau^{1}, \ldots, \tau^{K} \in \bar{\Omega}_{0}
$$

provide a basis of the submodule $\operatorname{Ker} \bar{\Omega}_{0} \subset \bar{\Omega}_{0}$

(recall that $\operatorname{Ker}^{2} \bar{\Omega}_{0}=\operatorname{Ker} \bar{\Omega}_{0}$ whence $\mathscr{L}_{D} \operatorname{Ker} \bar{\Omega}_{0} \subset \operatorname{Ker} \bar{\Omega}_{0}$ ) and moreover the classes of forms

$$
\pi^{1}, \ldots, \pi^{j_{0}} \in \bar{\Omega}_{0} \text { provide a basis of } \bar{\Omega}_{0} / \operatorname{Ker} \bar{\Omega}_{0}
$$

(recall that $D: \bar{\Omega}_{0} / \operatorname{Ker} \bar{\Omega}_{0} \rightarrow \bar{\Omega}_{1} / \bar{\Omega}_{0}$ is injective mapping), the classes of forms

$$
\begin{aligned}
& \pi^{j_{0}+1}, \ldots, \pi^{j_{1}} \in \bar{\Omega}_{1} \\
& \text { provide a basis of } \bar{\Omega}_{1} /\left(\bar{\Omega}_{0}+\mathscr{L}_{\mathscr{H}} \bar{\Omega}_{0}\right)
\end{aligned}
$$

(recall that $D: \bar{\Omega}_{1} / \bar{\Omega}_{0} \rightarrow \bar{\Omega}_{2} / \bar{\Omega}_{1}$ is injective mapping), and in general the classes of forms

$$
\begin{aligned}
& \pi^{j_{l-1}+1}, \ldots, \pi^{j_{l}} \in \bar{\Omega}_{l} \\
& \text { provide a basis of } \bar{\Omega}_{l} /\left(\bar{\Omega}_{l-1}+\mathscr{L}_{\mathscr{H}} \bar{\Omega}_{l-1}\right) .
\end{aligned}
$$

Alternatively saying, the following forms constitute a basis:

$$
\begin{aligned}
& \tau^{1}, \ldots, \tau^{K} \text { of } \operatorname{Ker} \bar{\Omega}_{0}, \\
& \text { together with } \pi^{1}, \ldots, \pi^{j_{0}} \text { of } \bar{\Omega}_{0}, \\
& \text { together with } \mathscr{L}_{D} \pi^{1}, \ldots, \mathscr{L}_{D} \pi^{j_{0}}, \pi^{j_{0}+1}, \ldots, \pi^{j_{1}} \text { of } \bar{\Omega}_{1}, \\
& \text { together with } \mathscr{L}_{D}^{2} \pi^{1}, \ldots, \mathscr{L}_{D}^{2} \pi^{j_{0}}, \mathscr{L}_{D} \pi^{j_{0}+1}, \ldots, \mathscr{L}_{D} \pi^{j_{1}}, \\
& \pi^{j_{1}+1}, \ldots, \pi^{j_{2}} \text { of } \bar{\Omega}_{2},
\end{aligned}
$$

and so on. Let us denote

$$
\pi_{r}^{j}=\mathscr{L}_{D}^{r} \pi^{j} \quad\left(j=j_{l-1}+1, \ldots, j_{l}\right) .
$$

In terms of this notation

$\tau^{1}, \ldots, \tau^{K}$ is a basis of $\operatorname{Ker} \bar{\Omega}_{0}$ and together with the forms

$$
\tau^{1}, \ldots, \tau^{K}
$$

$$
\begin{array}{rr}
\pi_{0}^{1}, \ldots, \pi_{0}^{j_{0}}, \quad \pi_{1}^{1}, \ldots, \pi_{1}^{j_{0}}, \ldots, & \pi_{l}^{1}, \ldots, \pi_{l}^{j_{0}}, \\
\pi_{0}^{j_{0}+1}, \ldots, \pi_{0}^{j_{1}}, \ldots, & \pi_{l-1}^{j_{0}+1}, \ldots, \pi_{l-1}^{j_{1}} \\
\ldots & \\
& \pi_{0}^{j_{l-1}+1}, \ldots, \pi_{0}^{j_{l}},
\end{array}
$$

we have the standard basis of $\bar{\Omega}_{l}$.
Clearly $j_{L}=j_{L+1}=\cdots$ and it follows that there is only a finite number $\mu(\Omega)=j_{L}$ of initial forms

$$
\begin{gathered}
\pi^{1}=\pi_{0}^{1}, \ldots, \pi^{j_{0}}=\pi_{0}^{j_{0}}, \pi^{j_{0}+1}=\pi_{0}^{j_{0}+1}, \\
\ldots, \pi^{j_{L-1}+1}=\pi_{0}^{j_{L-1}+1}, \ldots, \pi^{j_{L}}=\pi_{0}^{j_{L}}
\end{gathered}
$$

with the lower zero indice. The following forms $\pi_{r}^{j}(r>0)$ satisfy the recurrence and the (equivalent) congruence

$$
\mathscr{L}_{D} \pi_{r}^{j}=\pi_{r+1}^{j}, \quad d \pi_{r}^{j} \cong d x \wedge \pi_{r+1}^{j} \quad(\bmod \Omega \wedge \Omega) .
$$

In this sense, the linearly independent forms $\pi_{r}^{j}$ are generalizations of the classical contact forms $\omega_{r}^{j}=d w_{r}^{j}-$ $w_{r+1}^{j} d x$ of the jet theory.

Theorem 13. Let $\bar{\Omega}_{*}$ be a standard filtration of diffiety $\Omega$. Then the submodule $\operatorname{Ker} \bar{\Omega}_{0} \subset \Omega$ is generated by all differentials $d f \epsilon$ $\Omega$.

Proof. First assume $d f \in \Omega$. Then $D f=d f(D)=0$ whence $\mathscr{L}_{D} d f=d D f=0$. Clearly $d f \in \bar{\Omega}_{l}$ for appropriate $l$. This implies $d f \in \operatorname{Ker} \bar{\Omega}_{l-1}$, if $l \geq 0$ therefore $d f \in \operatorname{Ker} \bar{\Omega}_{0}$. It follows that $\operatorname{Ker} \bar{\Omega}_{0}$ contains all differentials $d f \in \Omega$.

Conversely let $\tau \in \operatorname{Ker} \bar{\Omega}_{0}$. Due to the equality $\operatorname{Ker} \bar{\Omega}_{0}=$ $\operatorname{Ker}^{2} \Omega_{0}$, we have $\mathscr{L}_{D} \tau \in \operatorname{Ker} \bar{\Omega}_{0}$ whence $d \tau \cong d x \wedge$ $\mathscr{L}_{D} \tau(\bmod \Omega \wedge \Omega)$, consequently

$$
d \tau \cong \sum a_{r s}^{j i} \pi_{r}^{j} \wedge \pi_{s}^{i} \quad\left(\bmod \operatorname{Ker} \bar{\Omega}_{0}, \text { sum over } i \leq j\right) .
$$

It follows that $a_{r s}^{j i}=0$ identically by using $d(d \tau)=0$ and (55). (Hint: look at assumed top order product $\pi_{R}^{j} \wedge \pi_{s}^{i}$ where $R \geq$ all $r$. Then $d^{2} \tau$ involves only one summand with $d x \wedge \pi_{R+1}^{j} \wedge \pi_{s}^{i}$ which is impossible since $d^{2}=0$.) Therefore $d\left(\operatorname{Ker} \bar{\Omega}_{0}\right) \cong 0\left(\bmod \operatorname{Ker} \bar{\Omega}_{0}\right)$ and the Frobenius theorem can be applied. Module $\operatorname{Ker} \bar{\Omega}_{0}$ has a basis consisting of total differentials.

Definition 14. We may denote $\mathscr{R}(\Omega)=\operatorname{Ker} \bar{\Omega}_{0}$ since this module does not depend on the choice of the filtration (22). Together with the original basis $\tau^{1}, \ldots, \tau^{K}$ occurring in (53), there exists alternative basis $d t^{1}, \ldots, d t^{K}$ with differentials. In the particular case $\mathscr{R}(\Omega)=0$, hence, $K=0$, we speak of a controllable diffiety $\Omega$.

Remark 15. The controllability is a familiar concept of the theory of underdetermined ordinary differential equations or Pfaffian systems in finite-dimensional spaces [12]; however, some aspects due to diffieties are worth mentioning here. If $\mathscr{R}(\Omega) \neq 0$ is a nontrivial module, the underlying space $\mathbf{M}$ is fibered by the leaves $t^{k}=c^{k} \in \mathbb{R}(k=1, \ldots, K)$ depending on $K>0$ parameters. A curve $\mathbf{p}: \mathbf{I} \rightarrow \mathbf{M}(\mathbf{I} \subset \mathbb{R})$ is called a solution of diffiety $\Omega$, if $\mathbf{p}^{*} \omega=0(\omega \in \Omega)$. Since $d t^{k} \in \mathscr{R}(\Omega) \subset \Omega$, we have

$$
\begin{array}{r}
\mathbf{p}^{*} d t^{k}=d \mathbf{p}^{*} t^{k}=0, \quad \mathbf{p}^{*} t^{k}=c^{k} \in \mathbb{R} \\
(k=1, \ldots, K),
\end{array}
$$




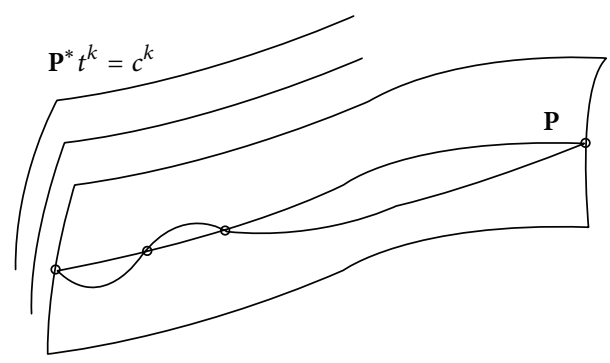

(a) Non-contrillable case

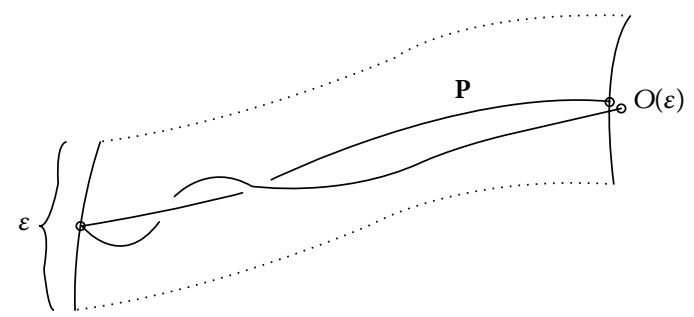

(b) Mayer extermal

Figure 2

therefore every solution of diffiety $\Omega$ is contained in a certain leaf (the Figure 2(a)).

In the controllable case, such foliation of the space $\mathbf{M}$ does not exist. However, the construction of the standard filtration need not be of the "universal nature." There may exist some "exceptional points" where the terms $\pi_{r}^{i}$ of the standard basis are not independent. We may even obtain a solution $\mathbf{p}$ consisting of such exceptional points and then there appears the "infinitesimal leaf" of the noncontrollability along $\mathbf{p}$ which means that $\mathbf{p}$ is a Mayer extremal (the Figure 2(b)). We refer to article [13] inspired by the beautiful paper [14]. In the present paper, such exceptional points are tacitly excluded. They produce singularities of the symmetry groups and deserve a special, not yet available approach. It should be noted that the noncontrollable case also causes some technical difficulties. We may however suppose $\mathscr{R}(\Omega)=0$ without much loss of generality since the noncontrollable diffiety can be restricted to a leaf and regarded as a diffiety depending on parameters $c^{1}, \ldots, c^{K}$.

Theorem 16. The total number $\mu(\Omega)$ of initial forms does not depend on the choice of the good filtration (22).

Proof. Filtration (22) differs from the standard filtration $\bar{\Omega}_{*}$ only in lower terms whence

$$
\operatorname{dim} \Omega_{l}=\operatorname{dim} \bar{\Omega}_{l}+\text { const. }=\mu(\Omega) l+\text { const. }
$$

( $l$ large enough) .

Let another filtration $\widetilde{\Omega}_{*}: \widetilde{\Omega}_{0} \subset \widetilde{\Omega}_{1} \subset \cdots \subset \Omega=\cup \widetilde{\Omega}_{l}$ of diffiety $\Omega$ provide (corresponding standard filtration and therefore) certain number $\widetilde{\mu}(\Omega)$ of (other) initial forms. Then

$$
\operatorname{dim} \Omega_{l}=\operatorname{dim} \widetilde{\Omega}_{l}+\text { const. }=\widetilde{\mu}(\Omega) l+\text { const. }
$$

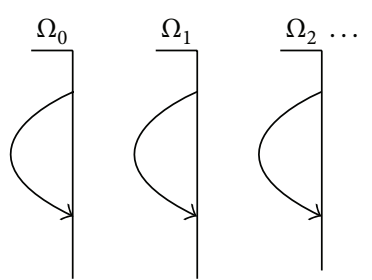

(a)

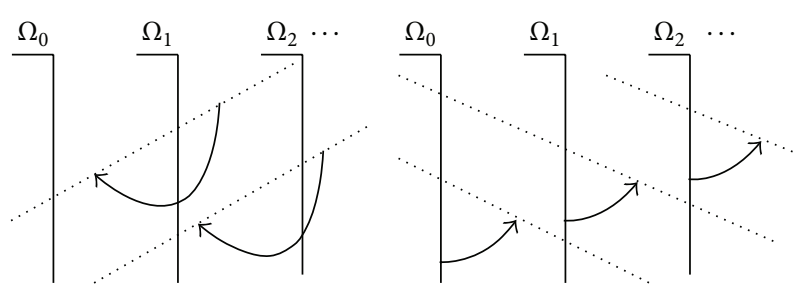

(b)

(c)

FIGURE 3

However $\Omega_{l} \subset \widetilde{\Omega}_{L(l)} \subset \Omega_{M(l)}$ for appropriate $L(l)$ and $M(l)$ whence

$$
\Omega_{l+k} \subset \widetilde{\Omega}_{L(l)+k} \subset \Omega_{M(l)+k} \quad(l, k \text { large enough })
$$

by using (23) and the equality $\mu(\Omega)=\widetilde{\mu}(\Omega)$ easily follows.

\section{On the Morphisms and Variations}

A huge literature on the point symmetries (scheme (a) of Figure 3, the order of derivatives is preserved) of differential equations is available. On the contrary, we can mention only a few fundamental principles for the generalized (or higherorder) symmetries (scheme (c) of Figure 3) since the general theory deserves quite another paper. Our modest aim is to clarify a little the mechanisms of the particular examples to follow. We will also deal with generalized (or higher-order) groups of symmetries and the relevant generalized infinitesimal symmetries (scheme (b) Figure 3) with ambiguous higherorder invariant subspaces (the dotted lines). Figure 3 should be therefore regarded as a rough description of the topics to follow and we also refer to Section 9 for more transparent details. The main difficulty of the higher-order theory lies in the fact that the dotted domains are not known in advance. Modules $\Omega_{l}$ represent the "natural" filtration with respect to the primary order of contact forms in the ambient jet space, see the examples. They depend on the accidental inclusion $\mathbf{M} \subset \mathbf{M}(m)$ mentioned in Section 1 and do not have any true geometrical sense in the internal approach. It is to be therefore surprisingly observed that the seemingly "exotic" at the first glance concept of higher-order transformations of Section 1 should be regarded for reasonable and the only possible in the coordinate free theory. On the other hand, an important distinction between the group-like morphisms with large number of finite-dimensional invariant subspaces (scheme (a) and (b)) and the genuine order-destroying morphisms without such subspaces (scheme (c)) is of the highest importance.

( $l$ large enough). 
We are passing to rigorous exposition. Let us recall the diffiety $\Omega \subset \Phi(\mathbf{M})$ on the space $\mathbf{M}$, the independent variable $x \in \mathscr{F}(\mathbf{M})$ with the corresponding vector field $D=D_{x} \in$ $\Omega^{\perp}=\mathscr{H}$, the controllability submodule $\mathscr{R}(\Omega) \subset \Omega$ with the basis $d t^{1}, \ldots, d t^{K}$, and a standard basis $\pi_{r}^{j}(j=$ $1, \ldots, \mu(\Omega) ; r=0,1, \ldots)$ of diffiety $\Omega$.

Let us begin with morphisms.

Lemma 17. If $\mathbf{m}: \mathbf{M} \rightarrow \mathbf{M}$ is a morphism of $\Omega$ then $\mathbf{m}^{*} \mathscr{R}(\Omega) \subset \mathscr{R}(\Omega)$ and the recurrence

$$
\begin{array}{r}
D W \mathbf{m}^{*} \pi_{r+1}^{j} \cong \mathscr{L}_{D} \mathbf{m}^{*} \pi_{r}^{j} \\
\left(W=\mathbf{m}^{*} x ; j=1, \ldots, \mu(\Omega) ; r=0,1, \ldots\right)
\end{array}
$$

modulo $\mathscr{R}(\Omega)$ holds true.

Proof. If $\mathbf{m}$ is a morphism then $\mathbf{m}^{*} \Omega \subset \Omega$ therefore $\mathbf{m}^{*} \mathscr{R}(\Omega) \quad \subset \quad \mathscr{R}(\Omega)$ (use Theorem 13) and $\mathbf{m}^{*} \pi_{r}^{j} \cong$ $\sum a_{r s}^{j i} \pi_{s}^{i}(\bmod \mathscr{R}(\Omega))$. It follows that

$$
\begin{aligned}
\mathbf{m}^{*} d \pi_{r}^{j} \cong & \mathbf{m}^{*}\left(d x \wedge \pi_{r+1}^{j}\right) \cong D W d x \wedge \mathbf{m}^{*} \pi_{r+1}^{j}, \\
d \mathbf{m}^{*} \pi_{r}^{j} \cong & d \sum a_{r s}^{j i} \pi_{s}^{i} \\
\cong & \sum D a_{r s}^{j i} d x \wedge \pi_{s}^{i} \\
& +\sum a_{r s}^{j i} d x \wedge \pi_{s+1}^{i} \cong d x \wedge \mathscr{L}_{D} \mathbf{m}^{*} \pi_{r}^{j}
\end{aligned}
$$

modulo $\mathscr{R}(\Omega)$ and $\Omega \wedge \Omega$. This implies (61) by comparing both factors of $d x$.

Remark 18. On this occasion, the following useful principles of calculation are worth mentioning:

$$
\begin{aligned}
& \text { if } \alpha, \beta \in \Omega \text { satisfy } d \alpha \cong d x \wedge \beta \quad(\bmod \Omega \wedge \Omega) \\
& \text { then } D W \mathbf{m}^{*} \beta=\mathscr{L}_{D} \mathbf{m}^{*} \alpha,
\end{aligned}
$$

if $u, v \in \mathscr{F}(\mathbf{M}), d u-v d x \in \Omega$ then $D W \mathbf{m}^{*} v=D \mathbf{m}^{*} u$,

and in general

$$
\mathbf{m}^{*} D f \cdot D \mathbf{m}^{*} g=\mathbf{m}^{*} D g \cdot D \mathbf{m}^{*} f \quad(f, g \in \mathscr{F}(\mathbf{M})) .
$$

In terms of notation (21), we conclude that $\mathbf{m}_{*} D_{\mathbf{P}}=D W(\mathbf{P})$. $D_{\mathrm{Q}}$ and therefore

$$
\mathbf{m}_{*}\left(\frac{1}{D W} D\right)=D \quad\left(W=\mathbf{m}^{*} x\right),
$$

if the morphism $\mathbf{m}$ of diffiety $\Omega$ is invertible.

Let us turn to invertible morphisms.

Lemma 19. The inverse of a morphism $\mathbf{m}$ again is a morphism.

Proof. Assume $\omega \in \Omega, \mathbf{m}^{-1^{*}} \omega \cong f d x(\bmod \Omega)$. Then

$$
\omega=\mathbf{m}^{*} \mathbf{m}^{-1^{*}} \omega \cong \mathbf{m}^{*}(f d x)=\mathbf{m}^{*} f \cdot d W \in \Omega,
$$

where $d W=d \mathbf{m}^{*} x=\mathbf{m}^{*} d x \neq 0$. Hence $\mathbf{m}^{*} f=0, f=0$ and therefore $\mathbf{m}^{-1^{*}} \Omega \subset \Omega$.
We have $\mathbf{m}^{*} \Omega \subset \Omega$ if $\mathbf{m}: \mathbf{M} \rightarrow \mathbf{M}$ is a morphism and moreover $\mathbf{m}^{-1 *} \Omega \subset \Omega$ hence $\Omega^{*} \subset \mathbf{m}^{*} \Omega$ in the invertible case. The converse and rather useful assertion is as follows.

Theorem 20. A morphism $\mathbf{m}$ of diffiety $\Omega$ is invertible if and only if $\mathbf{m}^{*} \Omega=\Omega$.

This may be obtained easily from the following result.

Lemma 21. Let $\mathbf{m}^{*} \mathscr{R}(\Omega)=\mathscr{R}(\Omega)$ and $\pi_{0}^{j} \in \mathbf{m}^{*} \Omega(j=$ $1, \ldots, \mu(\Omega))$. Then $\mathbf{m}$ is invertible.

Proof. Proof of the Lemma 21 is analogous as in [2, Theorem $2]$ and we briefly recall only the main principles here. It is sufficient to prove the invertibility of $\mathbf{m}^{*}: \Omega \rightarrow \Omega$.

Assuming $\pi_{r}^{j} \in \mathbf{m}^{*} \Omega$ then $\pi_{r+1}^{j}=\mathscr{L}_{D} \pi_{r}^{j} \in \mathbf{m}^{*} \Omega$ by virtue of recurrence (61). It follows that $\Omega \subset \mathbf{m}^{*} \Omega$ and $\mathbf{m}^{*}$ is surjective. We prove that $\mathbf{m}^{*}: \Omega \rightarrow \Omega$ is even injectivity by using the well-known algebraical interrelation between filtrations and gradations.

Let us introduce filtrations $\Omega_{*}\left(\bar{\Omega}_{*}\right.$, resp.) as follows: the submodule $\Omega_{l} \subset \Omega\left(\bar{\Omega}_{l} \subset \Omega\right)$ is generated by $\mathscr{R}(\Omega)$ and all forms $\pi_{r}^{j}\left(\mathbf{m}^{*} \pi_{r}^{j}\right)$ where $r \leq l$. We also introduce the gradations

$$
\begin{array}{rr}
\mathscr{M}=\oplus \mathscr{M}_{l} & \left(\mathscr{M}_{l}=\Omega_{l} / \Omega_{l-1}\right), \\
\overline{\mathscr{M}}=\oplus \overline{\mathscr{M}}_{l} & \left(\overline{\mathscr{M}}_{l}=\bar{\Omega}_{l} / \bar{\Omega}_{l-1}\right) \\
& (l=0,1, \ldots)
\end{array}
$$

(formally $\Omega_{-1}=\bar{\Omega}_{-1}=0$ ). It follows that the naturally induced mapping $\mathbf{m}^{*}: \mathscr{M} \rightarrow \mathscr{M}$ is surjective and it is sufficient to prove that this induced $\mathbf{m}^{*}$ is also injective.

We are passing to the most delicate part of the proof. The surjectivity of $\mathbf{m}^{*}: \Omega \rightarrow \Omega$ implies that $\Omega_{0} \subset \bar{\Omega}_{L}$ for $L$ large enough. Therefore $\Omega_{l} \subset \bar{\Omega}_{L+l}$ by applying the recursion (61) which implies

$$
\begin{array}{r}
\operatorname{dim} \Omega_{l}=\mu(\Omega) l+\text { const. } \leq \operatorname{dim} \bar{\Omega}_{L+l} \\
\left(\mu(\Omega)=\operatorname{dim} \mathscr{M}_{l} ; l=0,1, \ldots\right) .
\end{array}
$$

On the other hand, assume the noninjectivity therefore the existence of a nontrivial identity

$$
0=\sum a_{r}^{i} \mathbf{m}^{*} \pi_{r}^{i}=\cdots+\sum a_{R}^{i} \mathbf{m}^{*} \pi_{R}^{i} \quad \text { (top-order terms). }
$$

Then $0=\cdots+(D W)^{-l} \sum a_{R}^{i} \mathbf{m}^{*} \pi_{R+l}^{i}(l=0,1, \ldots)$ by applying operator $\mathscr{L}_{D}$ and recurrence (61). Due to the existence of such identities, it follows that

$$
\begin{gathered}
\operatorname{dim} \overline{\mathscr{M}}_{l}<\operatorname{dim} \mathscr{M}_{l}=\mu(\Omega), \\
\operatorname{dim} \bar{\Omega}_{L+l} \leq(\mu(\Omega)-1) l+\text { const. }
\end{gathered}
$$

and this is a contradiction. 
Remark 22. Recall that if $\mathbf{m}: \mathbf{M} \rightarrow \mathbf{M}$ is a mapping and $\Omega \subset \Phi(\mathbf{M})$ a submodule, then $\mathbf{m}^{*} \Omega \subset \Phi(\mathbf{M})$ denotes the submodule with generators $\mathbf{m}^{*} \omega(\omega \in \Omega)$ in accordance with the common practice in the algebraical module theory. Let in particular $\Omega$ be a diffiety and assume $\mathscr{R}(\Omega)=0$ for simplicity. Then module $\mathbf{m}^{*} \Omega$ is generated by all forms $\mathbf{m}^{*} \pi_{r}^{j}$ and therefore by all forms $\mathscr{L}_{D}^{r} \mathbf{m}^{*} \pi_{0}^{j}$, see Lemma 17. It follows that the invertibility of the morphism $\mathbf{m}$ depends only on the properties of the forms $\mathbf{m}^{*} \pi_{0}^{j}$, see Lemma 21 . In this sense, the invertibility problem is reduced to the finite-dimensional reasonings.

We turn to the variations.

Lemma 23. A vector field $Z \in \mathscr{T}(\mathbf{M})$ is a variation of diffiety $\Omega$ if and only if

$$
\pi_{r+1}^{j}(Z)=D \pi_{r}^{j}(Z) \quad(j=1, \ldots, \mu(\Omega) ; r=0,1, \ldots)
$$

and all $Z t^{k}(k=1, \ldots, K$; fixed $k)$ are functions only of variables $t^{1}, \ldots, t^{K}$.

Proof. We suppose $\mathscr{L}_{Z} \Omega \subset \Omega$ which is equivalent to the congruences

$$
\begin{aligned}
& \mathscr{L}_{Z} d t^{k}=d Z t^{k} \cong D Z t^{k} d x=0 \quad(\bmod \Omega), \\
& \left.\mathscr{L}_{Z} \pi_{r}^{j}=Z\right\rfloor d \pi_{r}^{j}+d \pi_{r}^{j}(Z) \\
& \cong\left(-\pi_{r+1}^{j}(Z)+D \pi_{r}^{j}(Z)\right) d x=0 \quad(\bmod \Omega)
\end{aligned}
$$

by using ((26) and (55)). So we have obtained (72) and moreover identities $D Z t^{k}=0(k=1, \ldots, K)$.

It is sufficient to prove that the latter identities imply $d Z t^{k}=0\left(\bmod d t^{1}, \ldots, d t^{K}\right)$. However, every differential $d f(f \in \mathscr{F}(\mathbf{M}))$ can be represented as

$$
d f=D f d x+\sum f^{k^{\prime}} d t^{k^{\prime}}+\sum f_{r}^{j} \pi_{r}^{j}
$$

in terms of the standard basis. Assuming in particular $f=$ $Z t^{k}$ (fixed $\left.k=1, \ldots, K\right)$, we have already obtained the equation $D f=0$ and then identities $f_{r}^{j}=0$ easily follow by applying the common rule $d(d f)=0$ together with (26). This concludes the proof.

Theorem 24. A variation $Z$ of diffiety $\Omega$ is infinitesimal symmetry of $\Omega$ if and only if all forms $\mathscr{L}_{Z}^{k} \pi_{0}^{j}(k=0,1, \ldots)$ are contained in a finite-dimensional module.

We omit lengthy proof and refer to more general results [5, Lemma 5.4, Theorem 5.6, and especially Theorem 11.1]. In future examples, we apply other and quite elementary arguments in order to avoid the nontrivial Theorem 24.

Remark 25. It follows from Lemma 23 that variations $Z$ of diffiety $\Omega$ can be represented by the universal series

$$
Z=\sum c^{k} \frac{\partial}{\partial d t^{k}}+z \frac{\partial}{\partial d x}+\sum D^{r} p^{j} \frac{\partial}{\partial \pi_{r}^{j}},
$$

where $c^{k}=c^{k}\left(t^{1}, \ldots, t^{K}\right)$ are arbitrary composed functions and $z=Z x, p^{j}=\pi_{0}^{j}(Z)$ are arbitrary functions in $\mathscr{F}(\mathbf{M})$. We have explicit formulae for all variations (in common terms, for all Lie-Bäcklund infinitesimal symmetries) of a given system of ordinary differential equations. Recall that these variations $Z$ need not generate any true group, and though the criterion in Theorem 24 is formally simple, it is not easy to be applied. Lemma 17 can be regarded as a counterpart to Lemma 23 since it ensures quite analogous result for the morphism $\mathbf{m}$ or, better saying, for the pullback $\mathbf{m}^{*}: \Phi(\mathbf{M}) \rightarrow \Phi(\mathbf{M})$ of a morphism. In more detail, the quite arbitrary choice of the initial terms $\mathbf{m}^{*} \pi_{0}^{j}$ of recurrence (61) is in principle possible but provides a mere formal result (corresponding to the formal nature of variations $Z$ ) and does not ensure the existence of true morphism $\mathbf{m}$. We may refer to articles $[2,3]$ where the formal part (the algebra) is distinguished from the nonformal part (the analysis) in the higher-order algorithms.

We conclude this Section with the only gratifying result [9, point $(\nu)$ on page 40$]$

Theorem 26. The standard filtration is unique in the case $\mu(\Omega)=1$.

Proof. Let us take a fixed filtration (22) and the corresponding standard filtration (46). Since $\mu(\Omega)=1$, we have only one initial form $\pi_{0}^{1}$ and therefore $\tau^{1}, \ldots, \tau^{K}, \pi_{0}^{1}, \ldots, \pi_{l}^{1}$ is a basis of $\bar{\Omega}_{l}$; see (53). Let us take another standard filtration $\widetilde{\Omega}_{*}$. Then the module $\widetilde{\Omega}_{0}$ has certain basis

$$
\begin{gathered}
\tau^{1}, \ldots, \tau^{K} \quad(\text { common forms }), \\
\widehat{\pi}_{0}^{1}=\sum a_{r} \pi_{r}^{1}=\cdots+a_{R} \pi_{R}^{1} \quad\left(a_{R} \neq 0, \text { top-order term }\right) .
\end{gathered}
$$

These forms together with all $\tilde{\pi}_{s}^{1}=\mathscr{L}_{D}^{s} \hat{\pi}_{0}^{1}=\cdots+a_{R} \pi_{R+s}^{1}(s \geq$ 0 ) generate the module $\Omega$ and this is possible only if $R=0$. We conclude that $\tilde{\pi}_{0}^{1}=a_{R} \pi_{R}^{1}=a_{0} \pi_{0}^{1}$ which implies $\widetilde{\Omega}_{0}=\bar{\Omega}_{0}$ hence $\widetilde{\Omega}_{l}=\bar{\Omega}_{l}$ for all $l$.

Remark 27. It follows that in the particular case $\mu(\Omega)=1$, every symmetry and infinitesimal symmetry preserves all terms of the (unique) standard filtration. So we have a large family of finite-dimensional subspaces of the underlying space $\mathbf{M}$ which are preserved too. The classical methods acting in finite-dimensional spaces uniquely determined in advance can be applied and are quite sufficient in this case $\mu(\Omega)=1$.

Remark 28. In more generality, one could also consider two diffieties $\Omega$ and $\widetilde{\Omega}$ on the underlying spaces $\mathbf{M}$ and $\widetilde{\mathbf{M}}$, respectively. Though we do not deal with the isomorphism problems of two diffieties $\Omega$ and $\widetilde{\Omega}$ here, let us mention that such isomorphism is defined as invertible mapping $\mathbf{m}$ : $\widetilde{\mathbf{M}} \rightarrow \mathbf{M}$ of underlying spaces satisfying $\mathbf{m}^{*} \Omega=\widetilde{\Omega}$. Quite equivalent "absolute equivalence" problem was introduced in [15] and resolved just for the case $\mu(\Omega)=\mu(\widetilde{\Omega})=1$ (in our terminology) by using finite-dimensional methods. We have discovered alternative approach here: the isomorphism 
m identifies the unique standard filtrations of $\Omega$ and of $\widetilde{\Omega}$. On this occasion, it is worth mentioning Cartan's pessimistic notice (rather unusual in his work) to the case $\mu(\Omega)>$ 1 : "Je dois ajourter que la géneralization de la théorie de l'equivalence absolu aux systémes differentiels dont la solution générale dépend de deux functions arbitraires d'un argument n'est pas immédiate et souléve d'asses grosses difficultiés." The same notice can be literally repeated also for the theory of the higher-order symmetries treated in this paper.

\section{The Order-Preserving Case of Infinitesimal Symmetries}

We are passing to the first example which intentionally concerns the well-known "towering" problem in order to examine our method reliably. Let us deal with infinitesimal symmetries of differential equation

$$
\frac{d^{2} u}{d x^{2}}=F\left(\frac{d v}{d x}\right)
$$

involving two unknown functions $u=u(x)$ and $v=v(x)$. In external theory, (77) is identified with the subspace $\mathbf{i}: \mathbf{M} \subset$ $\mathbf{M}(2)$ defined by the conditions

$$
\begin{aligned}
& D^{r}\left(u_{2}-F\right)=u_{r+2}-D^{r} F\left(v_{1}\right)=0 \\
& \left(r=0,1, \ldots ; D=\frac{\partial}{\partial x}+\sum u_{r+1} \frac{\partial}{\partial u_{r}}+\sum v_{r+1} \frac{\partial}{\partial v_{r}}\right)
\end{aligned}
$$

in the jet space $\mathbf{M}(2)$. We use simplified notation of coordinates and contact forms

$$
\begin{array}{r}
u_{r}=w_{r}^{1}, \quad v_{r}=w_{r}^{2}, \quad \alpha_{r}=\omega_{r}^{1}, \quad \beta_{r}=\omega_{r}^{2} \\
(r=0,1, \ldots)
\end{array}
$$

here. We are, however, interested in internal theory, that is, in the diffiety $\Omega$ corresponding to (77). Diffiety $\Omega$ appears if the contact forms

$$
\begin{array}{r}
\alpha_{r}=d u_{r}-u_{r+1} d x, \quad \beta_{r}=d v_{r}-v_{r+1} d x \\
(r=0,1, \ldots)
\end{array}
$$

are restricted to the subspace $\mathbf{i}: \mathbf{M} \subset \mathbf{M}(2)$. In accordance with the common practice, let us again simplify as

$$
u_{r}=\mathbf{i}^{*} u_{r}, \quad v_{r}=\mathbf{i}^{*} v_{r}, \quad \alpha_{r}=\mathbf{i}^{*} \alpha_{r}, \quad \beta_{r}=\mathbf{i}^{*} \beta_{r}
$$

the notation of the restrictions to $\mathbf{M}$, and moreover $D$ will be regarded as a vector field on $\mathbf{M}$ from now on.

Let us outline the lengthy path of future reasonings for the convenience of reader. We begin with preparatory points $(\iota)-(\iota \iota)$. The underlying space $\mathbf{M}$ together with the diffiety $\Omega$ is introduced and the standard basis $\pi_{0}, \pi_{1}, \ldots(\mu(\Omega)=$ 1 , abbreviation $\pi_{r}=\pi_{r}^{1}$ ) of diffiety $\Omega$ is determined. The standard basis is related to the "common" basis of $\Omega$ by means of formulae (93). We obtain explicit representation (99) for the variations $Z$ with two arbitrary functions $z=Z x$ and
$p=\pi_{0}(Z)$ as the final result. Variations $Z$ generating the true group (i.e., the infinitesimal symmetries $Z$ of $\Omega$ ) satisfy certain strong conditions discovered in points $(\iota v)$ and $(\nu)$. The conditions are expressed by the resolving system (107) and (108) or, alternatively, by (112)-(114) only in terms of the functions $p, D p, D^{2} p$, and $D^{3} p$. This rather complicated resolving system which does not provide any clear insight is equivalent to much simpler crucial requirements (121) or (125) on the actual structure of function $p$; see the central points $(\nu l)-(\nu l u)$. Then the subsequent points are devoted to the explicit solution of these equations (125). This is a mere technical task of traditional mathematical analysis and we omit comments at this place.

( 1 The diffiety. Let us introduce space $\mathbf{M}$ equipped with coordinates $x, u_{0}, u_{1}, v_{r}(r=0,1, \ldots)$. Then

$$
\begin{aligned}
& u_{r+2}=D^{r} F\left(v_{1}\right) \\
& \left(r=0,1, \ldots ; D=\frac{\partial}{\partial x}+u_{1} \frac{\partial}{\partial u_{0}}+F \frac{\partial}{\partial u_{1}}+\sum v_{r+1} \frac{\partial}{\partial v_{r}}\right)
\end{aligned}
$$

are merely composed functions. The forms

$$
\begin{array}{cc}
\alpha_{0}=d u_{0}-u_{1} d x, & \alpha_{1}=d u_{1}-F d x, \\
\beta_{r}=d v_{r}-v_{r+1} d x & (r=0,1, \ldots)
\end{array}
$$

provide a basis of the diffiety $\Omega$; however, all forms $\alpha_{r}=d u_{r}-$ $u_{r+1} d x(r=2,3, \ldots)$ are also lying in $\Omega$ as follows from the obvious rule:

$$
\mathscr{L}_{D} \alpha_{r}=\alpha_{r+1}, \quad \mathscr{L}_{D} \beta_{r}=\beta_{r+1} \quad(r=0,1, \ldots)
$$

and the inclusion $\mathscr{L}_{D} \Omega \subset \Omega$.

(u) Standard Filtration. There exists the "natural" filtration $\Omega_{*}$ of diffiety $\Omega$ with respect to the order: submodule $\Omega_{l} \subset \Omega$ involves the forms $\alpha_{r}, \beta_{r}$ with $r \leq l$. Alternatively saying, $\alpha_{0}, \beta_{0}$ is a basis of $\Omega_{0}$ and

$$
\alpha_{0}, \beta_{0}, \alpha_{1}, \beta_{1}, \beta_{2}, \ldots, \beta_{l} \text { is a basis of } \Omega_{l} \quad(l \geq 1) .
$$

Clearly $\operatorname{Ker} \Omega_{l+1}=\Omega_{l}$ if $l \geq 1$ as follows from (84). However,

$$
\begin{aligned}
\mathscr{L}_{D} \alpha_{1} & =\mathscr{L}_{D}\left(d u_{1}-F d x\right)=d F-D F d x \\
& =F^{\prime}\left(d v_{1}-v_{2} d x\right)=F^{\prime} \beta_{1} \in \Omega_{1} .
\end{aligned}
$$

(Figure 4(a)) therefore

$$
\begin{aligned}
\mathscr{L}_{D}\left(\alpha_{1}-F^{\prime} \beta_{0}\right) & =F^{\prime} \beta_{1}-D F^{\prime} \beta_{0}-F^{\prime} \mathscr{L}_{D} \beta_{0} \\
& =-D F^{\prime} \beta_{0} \in \Omega_{0} .
\end{aligned}
$$

Then $\alpha_{0}, \alpha=\alpha_{1}-F^{\prime} \beta_{1}, \beta_{0}$ may be taken for a basis of module Ker $\Omega_{1}$ (Figure $4(\mathrm{~b})$ ).

Moreover

$$
\begin{gathered}
\mathscr{L}_{D} \alpha_{0}=\alpha_{1}=\alpha+F^{\prime} \beta_{0}, \\
\mathscr{L}_{D} \alpha=-D F^{\prime} \beta_{0} \in \operatorname{Ker} \Omega_{1},
\end{gathered}
$$




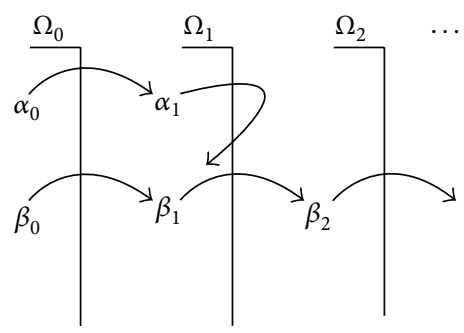

(a)

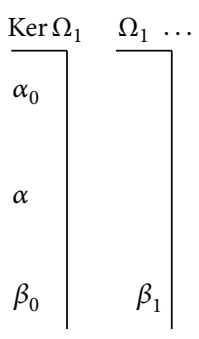

(b)

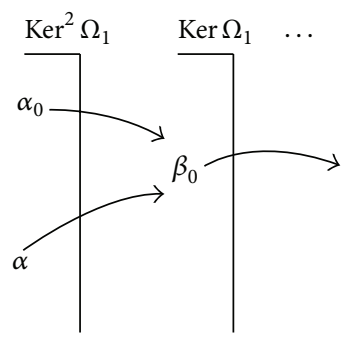

(c)
FIGURE 4

hence $\alpha_{0}, \alpha$ constitute a basis of module $\operatorname{Ker}^{2} \Omega_{1}$ (Figure 4(c)) and finally

$$
\begin{aligned}
& \mathscr{L}_{D}\left(F^{\prime} \alpha+D F^{\prime} \alpha\right) \\
& \quad=D F^{\prime} \alpha+D^{2} F^{\prime} \alpha_{0}+F^{\prime} \mathscr{L}_{D} \alpha+D F^{\prime} \mathscr{L}_{D} \alpha_{0} \\
& =2 D F^{\prime} \alpha+D^{2} F^{\prime} \alpha_{0} \in \operatorname{Ker}^{2} \Omega_{1} .
\end{aligned}
$$

Therefore assuming

$$
D F^{\prime}=F^{\prime \prime} v_{3} \neq 0 \quad\left(\text { hence } F^{\prime \prime} \neq 0\right)
$$

from now on, the form $\pi_{0}=F^{\prime} \alpha+D F^{\prime} \alpha_{0}$ may be taken for a basis of module $\operatorname{Ker}^{3} \Omega_{0}$. We have obtained the standard filtration

$$
\begin{aligned}
\bar{\Omega}_{*}: \bar{\Omega}_{0} & =\operatorname{Ker}^{3} \Omega_{1} \subset \bar{\Omega}_{1}=\operatorname{Ker}^{2} \Omega_{1} \subset \bar{\Omega}_{2} \\
& =\operatorname{Ker} \Omega_{1} \subset \bar{\Omega}_{3}=\Omega_{1} \subset \cdots \quad(\mathscr{R}(\Omega)=0),
\end{aligned}
$$

where forms

$$
\begin{aligned}
& \pi_{r}=\mathscr{L}_{D}^{r} \pi_{0} \\
& (r=0, \ldots, l ; \\
& \left.\pi_{0}=F^{\prime} \alpha+D F^{\prime} \alpha_{0}=D F^{\prime} \alpha_{0}+F^{\prime} \alpha_{1}-\left(F^{\prime}\right)^{2} \beta_{0}\right)
\end{aligned}
$$

provide a basis of module $\bar{\Omega}_{l}$.
Abbreviating $f=F^{\prime}$ from now on, explicit formulae

$$
\begin{gathered}
\pi_{0}=f \alpha+D f \alpha_{0}, \\
\pi_{1}=2 D f \alpha+D^{2} f \alpha_{0}, \\
\pi_{2}=3 D^{2} f \alpha+D^{3} f \alpha_{0}+C \beta_{0}, \\
C \alpha=D^{2} f \pi_{0}-D f \pi_{1}, \\
C \alpha_{0}=-2 D f \pi_{0}+f \pi_{1}, \\
C^{2} \beta_{0}=A \pi_{0}+B \pi_{1}+C \pi_{2},
\end{gathered}
$$

where $\alpha=\alpha_{1}-f \beta_{0}$ and

$$
\begin{gathered}
A=2 D f \cdot D^{3} f-3\left(D^{2} f\right)^{2}, \\
B=3 D f \cdot D^{2} f-f D^{3} f, \\
C=f D^{2} f-2(D f)^{2}
\end{gathered}
$$

can be easily found. They will be sufficient in calculations to follow. Recall that we suppose that the inequality (90) hold true, hence $C=\cdots+f f^{\prime} v_{3}=\cdots+F^{\prime} F^{\prime \prime} v_{3} \neq 0$.

(uil) Variations. We deal with vector fields

$$
Z=z \frac{\partial}{\partial x}+z_{0}^{1} \frac{\partial}{\partial u_{0}}+z_{1}^{1} \frac{\partial}{\partial u_{1}}+\sum z_{r}^{2} \frac{\partial}{\partial v_{r}}
$$

(the notation (75) with indices is retained) on the space M. Recall that $Z$ is a variation if $\mathscr{L}_{Z} \Omega \subset \Omega$. In terms of coordinates, the conditions are

$$
\begin{gathered}
z_{1}^{1}=D z_{0}^{1}-u_{1} D z, \\
f z_{0}^{1}=D z_{1}^{1}-F D z, \\
z_{r+1}^{2}=D z_{r}^{2}-v_{r+1} D z \quad(r=0,1, \ldots),
\end{gathered}
$$

where the first and third equations are merely recurrences while the middle equation causes serious difficulties (a classical result. Hint: use $\mathscr{L}_{Z} \alpha_{0} \in \Omega, \mathscr{L}_{Z} \alpha_{1} \in \Omega, \mathscr{L}_{Z} \beta_{r} \in$ $\Omega$ ). By using the alternative formula

$$
Z=z \frac{\partial}{\partial d x}+a_{0} \frac{\partial}{\partial \alpha_{0}}+a_{1} \frac{\partial}{\partial \alpha_{1}}+\sum b_{r} \frac{\partial}{\partial \beta_{r}},
$$

the conditions slightly simplify

$$
\begin{gathered}
a_{1}=D a_{0}, \quad D a_{1}=f b_{1}, \\
b_{r+1}=D b_{r} \quad(r=0,1, \ldots) .
\end{gathered}
$$

(Hint: apply the rule $\left.\mathscr{L}_{Z} \varphi=Z\right\rfloor d \varphi+d \varphi(Z)$ to the forms $\varphi=\alpha_{0}, \alpha_{1}, \beta_{r}$.) However, by virtue of Lemma 23 and standard filtration, we have explicit formula

$$
Z=z \frac{\partial}{\partial d x}+\sum D^{r} p \frac{\partial}{\partial \pi_{r}} \quad\left(z=Z x, p=\pi_{0}(Z)\right)
$$


for the variations where $z$ and $p$ are arbitrary functions. One can then easily obtain explicit formulae for all coefficients $a_{0}, a_{1}, b_{r}$ in (97) and $z_{0}^{1}, z^{1}, z_{r}^{2}$ in (95) by using the left-hand identities (93). They need not be stated here.

(ı) Infinitesimal Transformations. We refer to Remark 27: variation $Z$ is infinitesimal symmetry if and only if

$$
\left.\mathscr{L}_{Z} \pi_{0}=Z\right\rfloor d \pi_{0}+d p=\lambda \pi_{0}
$$

for appropriate multiplier $\lambda \in \mathscr{F}(\mathbf{M})$. In explicit terms, we recall formula

$$
\pi_{0}=f \alpha+D f \alpha_{0}=f \alpha+f^{\prime} v_{2} \alpha_{0},
$$

where

$$
\begin{gathered}
d \pi_{0}=d x \wedge \pi_{1} \quad(\bmod \Omega \wedge \Omega), \\
d \alpha=d\left(\alpha_{1}-f \beta_{0}\right) \cong-d f \wedge \beta_{0}=-f^{\prime} \beta_{1} \wedge \beta_{0}
\end{gathered}
$$

$(\bmod d x)$,

and therefore clearly

$$
\begin{aligned}
d \pi_{0}= & d x \wedge \pi_{1}+\left(f^{\prime} \beta_{1} \wedge \alpha-f f^{\prime} \beta_{1} \wedge \beta_{0}\right) \\
& +\left(f^{\prime \prime} v_{2} \beta_{1}+f^{\prime} \beta_{2}\right) \wedge \alpha_{0} \\
= & d x \wedge \pi_{1}+\beta_{1} \wedge\left(f^{\prime} \alpha-f f^{\prime} \beta_{0}+f^{\prime \prime} v_{2} \alpha_{0}\right) \\
& +f^{\prime} \beta_{2} \wedge \alpha_{0} .
\end{aligned}
$$

So denoting

$$
\begin{gathered}
z=Z x=d x(Z), \quad a_{0}=\alpha_{0}(Z), \quad a=\alpha(Z), \\
b_{r}=\beta_{r}(Z), \quad p=\pi_{0}(Z) \quad(r=0,1, \ldots),
\end{gathered}
$$

requirement (100) reads

$$
\begin{aligned}
z \pi_{1} & +b_{1}\left(f^{\prime} \alpha-f f^{\prime} \alpha_{0}+f^{\prime \prime} v_{2} \beta_{0}\right)+f^{\prime} b_{2} \alpha_{0} \\
& -\left(f^{\prime} a-f f^{\prime} a_{0}+f^{\prime \prime} v_{2} b_{0}\right) \beta_{1}-f^{\prime} a_{0} \beta_{2}+d p \\
= & \lambda\left(f \alpha+D f \alpha_{0}\right),
\end{aligned}
$$

where $\pi_{1}=2 D f \alpha+D^{2} f \alpha_{0}$ and

$$
\begin{aligned}
d p & \cong p_{u_{0}} \alpha_{0}+p_{u_{1}} \alpha_{1}+\sum p_{v_{r}} \beta_{r} \\
& =p_{u_{0}} \alpha_{0}+p_{u_{1}} \alpha+\left(f p_{u_{1}}+p_{v_{0}}\right) \beta_{0}+\sum_{r>0} p_{v_{r}} \beta_{r}
\end{aligned}
$$

$(\bmod d x)$ should be moreover inserted. It follows that requirement (100) is equivalent to the so-called resolving system

$$
\begin{gathered}
2 z D f+f^{\prime} b_{1}+p_{u_{1}}=\lambda f, \\
z D^{2} f+f^{\prime \prime} v_{2} b_{1}+p_{u_{0}}=\lambda D f, \\
f f^{\prime} b_{1}=f p_{u_{1}}+p_{v_{0}}, \\
f^{\prime} a-f f^{\prime} b_{0}+f^{\prime \prime} v_{2} a_{0}=p_{v_{1}}, \\
f^{\prime} a_{0}=p_{v_{2}} .
\end{gathered}
$$

Moreover $p_{v_{r}}=0(r \geq 2)$ and therefore $p=p\left(u_{0}, u_{1}\right.$, $\left.v_{0}, v_{1}, v_{2}\right)$ is of the order 2 at most.

(v) On the Resolving System. Equations (107) uniquely determine the multiplier $\lambda$ and the "horizontal" coefficient $z=$ $Z x$ in terms of the "vertical" coefficients $a_{0}, a, b_{r}$, and $p$. For instance the formula

$$
z=\frac{1}{C}\left(\left(f^{\prime} b_{1}+p_{u_{1}}\right) D f-\left(f^{\prime \prime} v_{2} b_{1}+p_{u_{0}}\right) f\right)
$$

easily follows. So we may focus on (108).

Equations (108) deserve more effort. They depend only on "vertical" components and can be expressed in terms of functions $p, D p, D^{2} p$, and $D^{3} p$ if the obvious identities

$$
\begin{gathered}
p=a f+a_{0} D f, \\
D p=2 a D f+a_{0} D^{2} f, \\
D^{2} p=3 a D^{2} f+a_{0} D^{3} f+b_{0} C, \\
C a=D^{2} f \cdot p-D f \cdot D p, \\
C a_{0}=-2 D f \cdot p+f D p, \\
C^{2} b_{0}=A p+B D p+C D^{2} p,
\end{gathered}
$$

following from (93) together with the prolongation formula

$$
C^{2} b_{1}+2 b_{0} C D C=D\left(C^{2} b_{0}\right)=D\left(A p+B D p+C D^{2} p\right)
$$

are applied. By using the lucky identity $D C=-B$ (direct verification), one can obtain the alternative resolving system

$$
\begin{gathered}
f f^{\prime}\left(C\left(D A \cdot p+(A+D B) D p+C D^{3} p\right)\right. \\
\left.+2 B\left(A p+B D p+C D^{2} p\right)\right) \\
=C^{3}\left(f p_{u_{1}}+p_{v_{0}}\right), \\
C\left(\left(f^{\prime} D^{2} f-2 v_{2} f^{\prime \prime} D f\right) p+\left(v_{2} f^{\prime \prime} f-f^{\prime} D f\right) D p\right) \\
-f f^{\prime}\left(A p+B D p+C D^{2} p\right) \\
=C^{2} p_{v_{1}}, \quad-2 f^{\prime} D f \cdot p+f f^{\prime} D p=C p_{v_{2}}
\end{gathered}
$$

only in terms of the unknown function $p$. Recall that the resolving system is satisfied if and only if the vector field (99) is infinitesimal symmetry.

Our aim is to determine the function $p$ satisfying (112)(114). Alas, the resolving system does not provide any insight into the true structure of function $p$. It will be therefore 
replaced by other conditions of classical nature, the crucial requirements and the simplified requirements as follows.

( vı) Crucial Requirements. We start with simple formulae

$$
\begin{gathered}
D f=f^{\prime} v_{2}, \quad D^{2} f=f^{\prime \prime} v_{2}^{2}+f^{\prime} v_{3}, \\
D^{3} f=f^{\prime \prime \prime} v_{2}^{3}+3 f^{\prime \prime} v_{2} v_{3}+f^{\prime} v_{4}, \\
D p=\cdots+p_{v_{2}} v_{3}, \quad D^{2} p=\cdots+p_{v_{2} v_{2}} v_{3}^{2}+p_{v_{2}} v_{4}
\end{gathered}
$$

(the top-order terms).

Using moreover (94), one can see that there is a unique summand in (113) which involves the factor $v_{3}^{3}$, namely the summand

$$
-f f^{\prime} \cdot C \cdot D^{2} p \cong-f f^{\prime} \cdot f f^{\prime} v_{3} \cdot p_{v_{2} v_{2}} v_{3}^{2} .
$$

It follows that $p_{v_{2} v_{2}}=0$ identically and we (temporarily) may denote

$$
p=M\left(x, u_{0}, u_{1}, v_{0}, v_{1}\right)+N\left(x, u_{0}, u_{1}, v_{0}, v_{1}\right) v_{2} .
$$

The simplest equation (114) of the resolving system then reads

$$
\begin{gathered}
-2 f^{\prime} \cdot f^{\prime} v_{2} \cdot\left(M+N v_{2}\right)+f f^{\prime} D\left(M+N v_{2}\right) \\
=\left(\left(f f^{\prime \prime}-2 f^{\prime 2}\right) v_{2}{ }^{2}+f f^{\prime} v_{3}\right) N .
\end{gathered}
$$

Clearly

$$
D\left(M+N v_{2}\right)=\mathscr{D} M+\left(M_{v_{1}}+\mathscr{D} N\right) v_{2}+N_{v_{1}} v_{2}^{2}+N v_{3} \text {, }
$$

where the reduced operator

$$
\mathscr{D}=\frac{\partial}{\partial x}+u_{1} \frac{\partial}{\partial u_{0}}+F \frac{\partial}{\partial u_{1}}+v_{1} \frac{\partial}{\partial v_{0}}
$$

appears and we obtain three so-called crucial requirements

$$
\mathscr{D} M=0, \quad 2 M f^{\prime}=\left(M_{v_{1}}+\mathscr{D N}\right) f, \quad N_{v_{1}} f^{\prime}=N f^{\prime \prime}
$$

for the functions $M, N$ by inspection of the variable $v_{2}$. Altogether taken, the last resolving equation (114) is equivalent to three requirements (121). We will see with great pleasure in ( $v u l$ ) below that requirements (121) ensure even the remaining equations (112) and (113) of the resolving system.

(vu) The Crucial Requirements Simplified. The right-hand equation (121) reads

$$
Q_{v_{1}}=0 \quad\left(Q=\frac{N}{f^{\prime}}\right)
$$

and the middle equation (121) reads

$$
f^{2} P_{v_{1}}+f^{\prime} \mathscr{D Q}=0 \quad\left(P=\frac{M}{f^{2}}\right),
$$

whence altogether

$$
p=P f^{2}+Q f^{\prime} v_{2}=P f^{2}+Q D f .
$$

The left-hand equation (121) does not change much; it may be expressed by $\mathscr{D} P=0$.

Let us summarize our achievements. In order to determine function $p$ given by (124), we have three simplified requirements

$$
\begin{aligned}
\mathscr{D} P & =0, \\
f^{2} P_{v_{1}}+f^{\prime} \mathscr{D} Q & =0, \quad Q_{v_{1}}=0
\end{aligned}
$$

for the coefficients $P=P\left(x, u_{0}, u_{1}, v_{0}, v_{1}\right)$ and $Q=Q\left(x, u_{0}\right.$, $\left.u_{1}, v_{0}, v_{1}\right)$.

( transcription (108) of the resolving system. We have already seen that (125) implies (114) and hence the equivalent and simplest right-hand equation (108).

Let us turn to the middle equation (108) equivalent to (113). One can directly find formulae

$$
\begin{aligned}
D p & =D P \cdot f^{2}+P D\left(f^{2}\right)+D Q \cdot D f+Q D^{2} f \\
& =P D\left(f^{2}\right)+Q D^{2} f
\end{aligned}
$$

by using (124) and (125). Moreover

$$
a=P f, \quad a_{0}=Q, \quad a_{1}=D a_{0}=\mathscr{D Q},
$$

$$
b_{0}=\frac{a_{1}-a}{f}=\frac{\mathscr{D Q}}{f}-P
$$

by using (124) and right-hand formulae (110). Substitution into middle equation (108) with

$$
p_{v_{1}}=\frac{\partial}{\partial v_{1}}\left(P f^{2}+Q f^{\prime} v_{2}\right)=P_{v_{1}} f^{\prime}+2 P f f^{\prime}+Q f^{\prime \prime} v_{2}
$$

gives the identity.

As the right-hand equation (108) equivalent to (112) is concerned, we may use

$$
\begin{gathered}
b_{1}=D b_{0}=\frac{\mathscr{D}^{2} Q}{f}+\frac{\partial}{\partial v_{1}}\left(\frac{\mathscr{D} Q}{f}\right) v_{2}-P_{v_{1}} v_{2}, \\
\mathscr{D}^{2} Q=-\mathscr{D}\left(\frac{f^{2}}{f^{\prime}} P_{v_{1}}\right),
\end{gathered}
$$

where

$$
\begin{gathered}
(\mathscr{D P})_{v_{1}}=\mathscr{D}\left(P_{v_{1}}\right)+P_{u_{1}} f+P_{v_{0}}=0, \\
(\mathscr{D Q})_{v_{1}}=Q_{u_{1}} f+Q_{v_{0}} .
\end{gathered}
$$

Moreover

$$
f p_{u_{1}}+p_{v_{0}}=f\left(P_{u_{1}} f^{2}+Q_{u_{1}} f^{\prime} v_{2}\right)+P_{v_{0}} f^{2}+Q_{v_{0}} f^{\prime} v_{2}
$$

and (108) again becomes the identity.

(ı) Back to the Crucial Requirements. Passing to the final part of this example, let us eventually solve (125) with the 
unknown functions $P, Q$ and given function $f$. This is already a task of classical mathematical analysis. We abbreviate $v=v_{1}$ from now on since this variable $v$ frequently occurs in our formulae.

Let us begin with middle equation (125) which reads

$$
P_{v}=\left(\frac{1}{f}\right)^{\prime} \cdot\left(q+F Q_{u_{1}}+v Q_{v_{0}}\right) \quad\left(q=Q_{x}+u_{1} Q_{u_{0}}\right)
$$

whence

$$
\begin{aligned}
P= & \frac{1}{f} q+\int\left(\frac{1}{f}\right)^{\prime} F d v \cdot Q_{u_{1}}+\int\left(\frac{1}{f}\right)^{\prime} v d v \cdot Q_{v_{0}} \\
& +\bar{P} \quad\left(\bar{P}=\bar{P}\left(x, u_{0}, u_{1}, v_{0}\right)\right)
\end{aligned}
$$

since $Q$ is independent of variable $v$ due to the right-hand equation (125). We may insert

$$
\begin{gathered}
\int\left(\frac{1}{f}\right)^{\prime} F d v=\frac{F}{f}-v, \\
\int\left(\frac{1}{f}\right)^{\prime} v d v=\frac{v}{f}-\int_{\bar{v}}^{v} \frac{d v}{f} \quad(\text { fixed } \bar{v} \in \mathbb{R})
\end{gathered}
$$

and the remaining left-hand equation (125) is expressed by the identity

$$
\begin{aligned}
1 \cdot( & \left.\bar{P}_{x}+u_{1} \bar{P}_{u_{0}}\right)+F \cdot \bar{P}_{u_{1}}+v \cdot \bar{P}_{v_{0}} \\
& +\frac{1}{f} \cdot\left(q_{x}+u_{1} q_{u_{0}}+F \cdot q_{u_{1}}+v \cdot q_{v_{0}}\right)+\left(\frac{F}{f}-v\right) \\
& \cdot\left(Q_{u_{1} x}+u_{1} Q_{u_{1} u_{0}}+F \cdot Q_{u_{1} u_{1}}+v \cdot Q_{u_{1} v_{0}}\right) \\
& +\left(\frac{v}{f}-\int \frac{d v}{f}\right) \cdot\left(q_{v_{0}}+F \cdot Q_{v_{0} u_{1}}+v \cdot Q_{v_{0} v_{0}}\right) \\
= & 0 .
\end{aligned}
$$

Functions $\bar{P}, q, Q$ are independent of $v$ and thereby subjected to very strong conditions by the inspection of the coefficients of functions

$$
\begin{aligned}
& 1, F, v, \frac{1}{f}, \frac{1}{f} F, \frac{1}{f} v,\left(\frac{F}{f}-v\right) F,\left(\frac{F}{f}-v\right) v, \\
& \frac{v}{f}-\int \frac{d v}{f},\left(\frac{v}{f}-\int \frac{d v}{f}\right) F,\left(\frac{v}{f}-\int \frac{d v}{f}\right) v
\end{aligned}
$$

in identity (135). The final result depends on the properties of function $F$ and we mention only a few instructive subcases here.

( $\kappa)$ The Generic Subcase. Functions (136) are in general linearly independent over $\mathbb{R}$ and identity (135) implies

$$
\begin{gathered}
\bar{P}_{x}+u_{1} \bar{P}_{u_{0}}=\bar{P}_{u_{1}}=\bar{P}_{v_{0}}-Q_{u_{1} x}-u_{1} Q_{u_{1} u_{0}}=0 \\
\left(q_{x}+u_{1} q_{u_{0}}\right)=Q_{x x}+2 u_{1} Q_{x u_{0}}+u_{1}^{2} Q_{u_{0} u_{0}}=0 \\
\left(q_{u_{1}}+Q_{u_{1} x}+u_{1} Q_{u_{1} u_{0}}\right)=Q_{u_{0}} \\
+2\left(Q_{u_{1} x}+u_{1} Q_{u_{1} u_{0}}\right)=0 \\
\left(q_{v_{0}}\right)=Q_{x v_{0}}+u_{1} Q_{u_{0} v_{0}}=Q_{u_{1} u_{1}} \\
=Q_{u_{1} v_{0}}=Q_{v_{0} v_{0}}=0 .
\end{gathered}
$$

The unknown functions $\bar{P}$ and $Q$ can be easily found as follows. We may suppose that

$$
\begin{gathered}
Q=a\left(x, u_{0}\right) u_{1}+b\left(x, u_{0}\right) v_{0}+c\left(x, u_{0}\right), \\
b\left(x, u_{0}\right)=B \in \mathbb{R}
\end{gathered}
$$

by using (140). Then $\bar{P}_{x}=\bar{P}_{u_{0}}=\bar{P}_{u_{1}}=0$; hence $\bar{P}=\bar{P}\left(v_{0}\right)$ due to (139). Moreover $\bar{P}^{\prime}=a_{x}+u_{1} a_{0}$ which implies $\bar{P}^{\prime}=$ $a_{x} \in \mathbb{R}, a_{0}=0$; hence $a=a(x)$ and altogether

$$
a=A x+\bar{A}, \quad \bar{P}=A v_{0}+C \quad(A, \bar{A}, C \in \mathbb{R}) .
$$

Then

$$
c=C_{1} x+C_{2} u_{0}+C_{3} \quad\left(C_{1}, C_{2}, C_{3} \in \mathbb{R}\right)
$$

follows from (138). Hence, $C_{2}+2 A=0$ due to (139) and altogether

$$
\begin{gathered}
Q=(A x+\bar{A}) u_{1}+B v_{0}+C_{1} x-2 A u_{0}+C_{3}, \\
\bar{P}=A v_{0}+C .
\end{gathered}
$$

Recalling moreover (133), we have explicit formulae for the solutions $P, Q$ of crucial requirements (125) and the symmetry problem is resolved. While $\bar{P}$ and $Q$ are mere polynomials, the total coefficient $P$ given by (133) depends on the quadrature $\int(d v / f)$ and this may be globally rather complicated function. It follows that, in our approach, the elementary and the "transcendental" parts of the solution are in a certain sense separated.

( $\iota)$ A Special Case of Function F. Let us choose $F(v)=e^{v}$. Then series (136) becomes quite explicit; namely,

$$
\begin{aligned}
& 1, e^{v}, v, e^{-v}, 1, v e^{-v},(1-v) e^{v}, \\
& \quad(1-v) v, v+1,(v+1) e^{v},(v+1) v e^{v}
\end{aligned}
$$

and these functions are linearly dependent. Identity (135) implies smaller number of requirements; the first term in (137) is combined with (139) into the single equation

$$
\bar{P}_{x}+u_{1} \bar{P}_{u_{0}}+q_{u_{1}}+Q_{u_{1} x}+u_{1} Q_{u_{1} u_{0}}=0
$$


without any other change. We can state the final solution

$$
\begin{gathered}
Q=(A x+\bar{A}) u_{1}+B v_{0}+C_{1} x+C_{2} u_{0}+C_{3}, \\
\bar{P}=A v_{0}+\left(C_{2}+2 A\right) x+C
\end{gathered}
$$

with only one additional parameter $C_{2} \in \mathbb{R}$ if compared to the previous formulae (144).

(кu) Another Special Case. Let us eventually mention the very prominent function $F(v)=v^{1 / 2}$; see $[1,7,16]$. Then the series

$$
1, v^{1 / 2}, v, 2 v^{1 / 2}, 2 v, 2 v^{3 / 2}, v^{3 / 2}, v^{2}, \frac{2}{3} v^{3 / 2}, \frac{2}{3} v^{2}, \frac{2}{3} v^{5 / 2}
$$

stands for (136) and the relevant identity (135) implies the system of equations

$$
\begin{gathered}
\bar{P}_{x}+u_{1} \bar{P}_{u_{0}}=0 \\
\bar{P}_{u_{1}}+2\left(q_{x}+u_{1} q_{u_{0}}\right) \\
=\bar{P}_{u_{1}}+2\left(Q_{x x}+2 u_{1} Q_{x u_{0}}+u_{1}^{2} Q_{u_{0} u_{0}}\right)=0 \\
\bar{P}_{v_{0}}+2 q_{u_{1}}+Q_{u_{1} x}+u_{1} Q_{u_{1} u_{0}} \\
=\bar{P}_{v_{0}}+2 Q_{u_{0}}+3\left(Q_{u_{1} x}+u_{1} Q_{u_{1} u_{0}}\right)=0 \\
6 q_{v_{0}}+3 Q_{u_{1} u_{1}}+2\left(Q_{v_{0} x}+u_{1} Q_{v_{0} u_{0}}\right) \\
=3 Q_{u_{1} u_{1}}+8\left(Q_{v_{0} x}+u_{1} Q_{v_{0} u_{0}}\right)=0, \\
Q_{u_{1} v_{0}}=0, \quad Q_{v_{0} v_{0}}=0 .
\end{gathered}
$$

We are passing to the solution of the system of (149)-(153) with unknown functions $Q=Q\left(x, u_{0}, u_{1}, v_{0}\right)$ and $\bar{P}=$ $\bar{P}\left(x, u_{0}, u_{1}, v_{0}\right)$. Due to (153), we may put

$$
Q=a\left(x, u_{0}\right) v_{0}+b\left(x, u_{0}, u_{1}\right)
$$

and then (152) is expressed by $3 b_{u_{1} u_{1}}+8\left(a_{x}+u_{1} a_{u_{0}}\right)=0$, whence easily

$$
b=-\frac{4}{9} a_{u_{0}} u_{1}^{3}-\frac{4}{3} a_{x} u_{1}^{2}+\bar{b}\left(x, u_{0}\right) u_{1}+\tilde{b}\left(x, u_{0}\right) .
$$

Moreover (151) reads $\bar{P}_{v_{0}}+2\left(a_{u_{0}} v_{0}+b_{u_{0}}\right)+3\left(b_{u_{1} x}+u_{1} b_{u_{1} u_{0}}\right)=0$, whence

$$
\begin{aligned}
\bar{P}= & -a_{u_{0}} v_{0}^{2}-\left(2 b_{u_{0}}+3\left(b_{u_{1} x}+u_{1} b_{u_{1} u_{0}}\right)\right) v_{0} \\
& +\bar{p}\left(x, u_{0}, u_{1}\right) .
\end{aligned}
$$

Remaining equations (149) and (150) do not admit such simple discussion. Using (154) and (155), identity (149) is equivalent to the system

$$
\begin{gathered}
a_{u_{0} x}+u_{1} a_{u_{0} u_{0}}=0 \\
\left(\text { whence } a=A u_{0}+\bar{a}(x), A \in \mathbb{R}\right), \\
2 b_{u_{0} x}+3\left(b_{u_{1} x x}+u_{1} b_{u_{1} u_{0} x}\right) \\
+u_{1}\left(2 b_{u_{0} u_{0}}+3\left(b_{u_{1} x u_{0}}+u_{1} b_{u_{1} u_{0} u_{0}}\right)\right)=0, \\
\bar{p}_{x}+u_{1} \bar{p}_{u_{0}}=0
\end{gathered}
$$

of three equations and identity (150) is equivalent to the system

$$
\begin{array}{r}
2 b_{u_{0} u_{1}}+3\left(b_{u_{1} x u_{1}}+b_{u_{1} u_{0}}+u_{1} b_{u_{1} u_{0} u_{1}}\right)=2 \bar{a}^{\prime \prime}, \\
\bar{p}_{u_{1}}+2\left(b_{x x}+2 u_{1} b_{x u_{0}}+u_{1}^{2} b_{u_{0} u_{0}}\right)=0
\end{array}
$$

if (157) is moreover employed. At the same time, (155) can be improved as

$$
b=-\frac{4}{9} A u_{1}^{3}-\frac{4}{3} \bar{a}^{\prime} u_{1}^{2}+\bar{b}\left(x, u_{0}\right) u_{1}+\widetilde{b}\left(x, u_{0}\right) .
$$

With this improvement, (160) reads $2 \bar{b}_{u_{0}}+3\left(-(8 / 4) \bar{a}^{\prime \prime}+\bar{b}_{u_{0}}\right)=$ $2 \bar{a}^{\prime \prime}$ and it follows that

$$
\bar{b}=2 \bar{a}^{\prime \prime} u_{0}+\widehat{b}(x)
$$

Analogously (158) reads

$$
\begin{aligned}
& 2\left(\bar{b}_{u_{0} x} u_{1}+\widetilde{b}_{u_{0} x}\right)+3\left(-\frac{8}{3} \bar{a}^{\prime \prime \prime} u_{1}+\bar{b}_{x x}+u_{1} \bar{b}_{u_{0} x}\right) \\
& +u_{1}\left(2 \widetilde{b}_{u_{0} u_{0}}+3 \bar{b}_{u_{0} x}\right)=0,
\end{aligned}
$$

which is equivalent to the system

$$
2 \widetilde{b}_{u_{0} x}+6 \bar{a}^{(4)} u_{0}+3 \widehat{b}^{\prime \prime}=0, \quad 4 \bar{a}^{\prime \prime \prime}+\widetilde{b}_{u_{0} u_{0}}=0,
$$

if (163) is inserted. Altogether, it follows that (158) is equivalent to

$$
\begin{gathered}
2 \widetilde{b}=-3 \bar{a}^{\prime \prime \prime} u_{0}^{2}-3 \widehat{b}^{\prime} u_{0}+\widetilde{b}_{0}\left(u_{0}\right)+\widetilde{b}_{1}(x), \\
\bar{a}^{\prime \prime \prime}=-\frac{1}{2} \widetilde{b}_{0}^{\prime \prime}=A_{3} \in \mathbb{R},
\end{gathered}
$$

whence

$$
\begin{aligned}
& \bar{a}=A_{3} \frac{x^{3}}{6}+A_{2} x^{2}+A_{1} x+A_{0}, \\
& \tilde{b}_{0}=-A_{3} u_{0}^{2}+B_{1} u_{0}+B_{0} \\
& \quad\left(A_{2}, A_{1}, A_{0}, B_{1}, B_{0} \in \mathbb{R}\right) .
\end{aligned}
$$

At the same time, we have improvements

$$
\begin{gathered}
\bar{b}=2\left(A_{3}+2 A_{2}\right) u_{0}+\widehat{b} \\
2 \widetilde{b}=-3 A_{3} u_{0}^{2}-3 \widehat{b}^{\prime} u_{0}+\widetilde{b}\left(u_{0}\right)+\widetilde{b}_{1}(x)
\end{gathered}
$$

of the above formulae. Let us eventually turn to the remaining equations (159) and (161). We begin with (161) which can be simplified to

$$
\bar{p}_{u_{1}}+2 \frac{16}{3} A_{3} u_{1}^{2}+2 \widehat{b}^{\prime \prime} u_{1}-\left(3 \widehat{b}^{\prime \prime \prime} u_{0}+\widetilde{b}_{1}^{\prime \prime}\right)=0
$$

whence

$$
\begin{aligned}
\bar{p}= & 2 \frac{16}{3} A_{3} \frac{u_{1}^{3}}{3}+\widehat{b}^{\prime \prime} u_{1}^{2}-\left(3 \widehat{b}^{\prime \prime \prime} u_{0}+\widetilde{b}_{1}^{\prime \prime}\right) u_{1} \\
& +\breve{p}\left(x, u_{0}\right) .
\end{aligned}
$$


Then the last requirement (159) is easily simplified as

$$
\begin{gathered}
\widehat{b}^{\prime \prime \prime} u_{1}^{2}-\left(3 \widehat{b}^{(4)} u_{0}+\widetilde{b}_{1}^{\prime \prime \prime}\right) u_{1}+\breve{p}_{x} \\
+u_{1}\left(-3 \widehat{b}^{\prime \prime \prime} u_{1}+\breve{p}_{u_{0}}\right)=0
\end{gathered}
$$

and it follows that

$$
\breve{p}_{x}=0, \quad \widetilde{b}_{1}^{\prime \prime \prime}-3 \widehat{b}^{(4)}+\breve{p}_{u_{0}}=0, \quad \widehat{b}^{\prime \prime \prime}=0,
$$

whence easily

$$
\begin{gathered}
\breve{p}=\breve{p}\left(u_{0}\right), \quad \tilde{b}_{1}^{\prime \prime \prime}=-\breve{p}^{\prime}=C_{3} \in \mathbb{R}, \\
\widehat{b}=D_{2} x^{2}+D_{1} x+D_{0}, \\
\widetilde{b}_{1}=C_{3} \frac{x^{3}}{6}+C_{2} x^{2}+C_{1} x+C_{0}, \\
\breve{p}=-C_{3} u_{0}+C \\
\left(C_{3}, D_{2}, \ldots, C \in \mathbb{R}\right) .
\end{gathered}
$$

The solution is eventually done. It depends on the parameters

$$
A, A_{3}, A_{2}, A_{1}, A_{0}, B_{1}, B_{0}, C_{3}, C_{2}, C_{1}, C_{0}, C, D_{2}, D_{1}, D_{0} \in \mathbb{R}
$$

in the total number of 15 . This is seemingly in contradiction with $[1,7,16]$ where 14 -dimensional symmetry group (namely, the exceptional simple Lie group $\mathbb{G}_{2}$ ) was declared. However, our final symmetry in fact depends on the sum $B_{0}+C_{0}$ as follows from (166), (167), and (174) and therefore no contradiction appears. We will not explicitly state the resulting symmetries $Z$ for obvious reason here. Recall that they are given by (99) where $z, p$ are clarified in (109) and (124). Coefficients appearing in (124) are clarified in (133), (156), and (170) and in (154), (157), (162), (168), (173), and (174).

It should be moreover noted that our approach is of the universal nature while the method of explicit calculations which provides the infinitesimal transformations in [7] rests on a lucky accident; see [7, Theorem 3.2, and the subsequent discussion].

Remark 29. Variations $Z$ were easily found in ( $\iota \iota)$. Due to Theorem 26 and Remark 27, infinitesimal symmetries satisfy moreover $\mathscr{L}_{Z} \pi_{0}=\lambda \pi_{0}$ or, alternatively saying, they preserve the Pfaffian equation $\pi_{0}=0$, and this property was just employed. We will now prove the converse without use of Theorem 24. The reasoning is as follows. Let a variation $Z$ preserve Pfaffian equation $\pi_{0}=0$. Then $Z$ preserves the space of adjoint variables $x, u_{0}, u_{1}, v_{0}, v_{1}$ of this Pfaffian equation. In this finite-dimensional space, the variation $Z$ generates a group which can be prolonged to the higher-order jet variables. It follows that $Z$ is indeed an infinitesimal transformation.
Remark 30. Let us briefly mention the case $F\left(v_{1}\right)=A v_{1}+$ $B(A, B \in \mathbb{R} ; A \neq 0)$ as yet excluded by condition (90). In this linear case, clearly

$$
\begin{gathered}
\mathscr{L}_{D} \alpha_{1}=\mathscr{L}_{D}\left(d u_{1}-\left(A v_{1}+B\right) d x\right) \\
=d\left(A v_{1}+B\right)-A v_{2} d x=A \beta_{1}, \\
\mathscr{L}_{D}\left(\alpha_{1}-A \beta_{0}\right)=0, \\
\tau=\alpha_{1}-A \beta_{0}=d\left(u_{1}-A v_{0}-B x\right) \in \mathscr{R}(\Omega)
\end{gathered}
$$

and we may introduce standard filtration

$$
\begin{aligned}
\mathscr{R}(\Omega) & \subset \bar{\Omega}_{0}=\operatorname{Ker}^{2} \Omega_{1} \subset \bar{\Omega}_{1} \\
& =\operatorname{Ker} \Omega_{1} \subset \bar{\Omega}_{0}=\Omega_{1} \subset \bar{\Omega}_{1}=\Omega_{2} \subset \cdots,
\end{aligned}
$$

where $\tau$ is a basis of $\mathscr{R}(\Omega)$ and the forms

$$
\begin{gathered}
\pi_{0}=\alpha_{0}, \quad \pi_{1}=\alpha_{1}, \\
\pi_{2}=\mathscr{L}_{D} \alpha_{1}=A \beta_{1}, \ldots, \pi_{l}=A \beta_{l-1}
\end{gathered}
$$

provide a basis of module $\bar{\Omega}_{l}(l \geq 1)$. The symmetries can be easily found. They are the prolonged contact transformations $\mathbf{m}$ defined by $\mathbf{m}^{*} \alpha_{0}=\lambda \alpha_{0}$ depending moreover on the parameter $t=u_{1}-A v_{0}-B x$. Roughly saying, the geometry of the linear second-order equation $u_{2}=A v_{1}+B$ is identical with the contact geometry of curves in $\mathbb{R}^{2}$. Quite analogous result can be obtained also for the Monge equation $F\left(x, u_{0}, u_{1}, v_{0}, v_{1}\right)=0$ and, in much greater generality, for the system of two Pfaffian equations in four-dimensional space [17].

Remark 31. Let us once more return to the crucial requirement (125) where operators $\mathscr{D}$ and $\partial / \partial v_{1}$ are applied to unknown functions $P$ and $Q$. We have employed the simplicity of the second operator $\partial / \partial v_{1}$ in the above solution; see formula (133). However, analogous "complementary" method can be applied to the first operator $\mathscr{D}$ as follows. Let us introduce new variables

$$
\begin{gathered}
\bar{x}=x, \quad \bar{u}_{0}=u_{0}-u_{1} x+\frac{F x^{2}}{2}, \\
\bar{u}_{1}=u_{1}-F x, \quad \bar{v}_{0}=v_{0}-v_{1} x, \quad \bar{v}=v_{1}
\end{gathered}
$$

with the obvious inverse transformation (not stated here). Then

$$
\begin{gathered}
\mathscr{D}=\frac{\partial}{\partial \bar{x}} \\
\frac{\partial}{\partial v_{1}}=\frac{f}{2} \bar{x}^{2} \frac{\partial}{\partial \bar{u}_{0}}-\bar{x}\left(f \frac{\partial}{\partial \bar{u}_{1}}+\frac{\partial}{\partial \bar{v}_{0}}\right)+\frac{\partial}{\partial \bar{v}} \\
\left(f=f\left(v_{1}\right)=f(\bar{v})\right)
\end{gathered}
$$

in terms of new variables. We again abbreviate $v=\bar{v}=v_{1}$. Passing to new coordinates, the left-hand requirement (125) 
is simplified as $P=\bar{P}\left(\bar{u}_{0}, \bar{u}_{1}, \bar{v}_{0}, v\right)$. The middle requirement (125) reads

$$
\begin{array}{r}
f^{2}\left(\frac{f}{2} \bar{x}^{2} \bar{P}_{\bar{u}_{0}}-\bar{x}\left(f \bar{P}_{\bar{u}_{1}}+\bar{P}_{\bar{v}_{0}}\right)+\bar{P}_{v}\right)+f^{\prime} \bar{Q}_{\bar{x}}=0 \\
\left(Q=\bar{Q}\left(\bar{x}, \bar{u}_{0}, \bar{u}_{1}, \bar{v}_{0}, v\right)\right)
\end{array}
$$

and determines the function $\bar{Q}$ in terms of new variables as

$$
\begin{gathered}
\bar{Q}=-\frac{f^{2}}{f^{\prime}}\left(\frac{f}{6} \bar{x}^{3} \bar{P}_{\bar{u}_{0}}-\frac{\bar{x}^{2}}{2}\left(f \bar{P}_{\bar{u}_{1}}+\bar{P}_{\bar{v}_{0}}\right)+\bar{x} \bar{P}_{v}\right)+\bar{q}, \\
\bar{q}=\bar{q}\left(\bar{u}_{0}, \bar{u}_{1}, \bar{v}_{0}, v\right),
\end{gathered}
$$

where $\bar{q}$ is constant of integration. This is a polynomial in variable $\bar{x}$ and it follows easily that the remaining right-hand requirement (130) applied to function $\bar{Q}$ is equivalent to the system

$$
\begin{aligned}
& \bar{P}_{\bar{u}_{0} \bar{u}_{0}}=0, \quad \mathscr{P}_{\bar{u}_{0}}=0, \quad \bar{q}_{v}=0, \\
& f \bar{q}_{\bar{u}_{1}}+\bar{q}_{\bar{v}_{0}}+\frac{\partial}{\partial v}\left(\frac{f^{2}}{f^{\prime}} \bar{P}_{v}\right)=0, \\
& \frac{f^{3}}{f^{\prime}} \bar{P}_{\bar{u}_{0} v}+\frac{f^{2}}{f^{\prime}}\left(f \mathscr{P}_{\bar{u}_{1}}+\mathscr{P}_{\bar{v}_{0}}\right)+\frac{1}{3} \frac{\partial}{\partial v}\left(\frac{f^{3}}{f^{\prime}} \overline{\bar{u}}_{\bar{u}_{0}}\right) \\
& =0 \text {, } \\
& f \bar{q}_{\bar{u}_{0}}+2 \frac{f^{2}}{f^{\prime}} \mathscr{P}_{v}+\frac{\partial}{\partial v}\left(\frac{f^{2}}{f^{\prime}} \mathscr{P}\right)=0 \\
& \left(\mathscr{P}=f \bar{P}_{\bar{u}_{1}}+\bar{P}_{\bar{v}_{0}}\right) \text {. }
\end{aligned}
$$

We will not discuss this alternative approach here in more detail.

Remark 32. Though the symmetries of (77) can be completely determined by applying the common methods, several formally quite different ways of the calculation are possible. It would certainly be of practical interest which of them is the "most economical" one. Let us mention such an alternative way for better clarity. We start with the "opposite" transcription

$$
\frac{d v}{d x}=G\left(\frac{d^{2} u}{d x^{2}}\right) \quad\left(G=F^{-1}, \text { the inverse function }\right)
$$

of (77). The primary concepts are retained, the same underlying space $\mathbf{M}$, diffiety $\Omega$, and contact forms $\alpha_{r}, \beta_{r}(r=$ $0,1, \ldots)$. However, we choose $x, u_{0}, u_{1}, \ldots, v_{0}$ for new coordinates on $\mathbf{M}$ from now on and the forms

$$
\begin{gathered}
\alpha_{r}=d u_{r}-u_{r+1} d x \quad(r=0,1, \ldots), \\
\beta_{0}=d v_{0}-G\left(u_{2}\right) d x
\end{gathered}
$$

for new basis of $\Omega$. We have moreover

$$
D=\frac{\partial}{\partial x}+\sum u_{r+1} \frac{\partial}{\partial u_{r}}+G \frac{\partial}{\partial v_{0}}
$$

in terms of new coordinates. The standard filtration is formally simplified. The forms

$$
\begin{aligned}
& \pi_{r}=\mathscr{L}_{D}^{r} \pi_{0} \\
& \left(r=0,1, \ldots ; \pi_{0}=\beta_{0}-G^{\prime} \alpha_{1}+D G^{\prime} \alpha_{0}\right)
\end{aligned}
$$

may be taken for new standard basis if the inequality $D^{2} G^{\prime} \neq 0$ is supposed. This follows from the obvious formulae

$$
\begin{gathered}
\pi_{1}=\mathscr{L}_{D} \pi_{0}=D^{2} G^{\prime} \alpha_{0}, \\
\pi_{2}=\mathscr{L}_{D} \pi_{1}=D^{3} G^{\prime} \alpha_{0}+D^{2} G^{\prime} \alpha_{1}, \ldots,
\end{gathered}
$$

simplifying the analogous left-hand side (93). Then, analogously to (95) and (99), we introduce the variations

$$
\begin{aligned}
Z= & z \frac{\partial}{\partial x}+\sum z_{r}^{1} \frac{\partial}{\partial u_{r}}+z_{0}^{2} \frac{\partial}{\partial v_{0}}=z \frac{\partial}{\partial d x} \\
& +\sum D^{r} p \frac{\partial}{\partial \pi_{r}}
\end{aligned}
$$

of diffiety $\Omega$ where $z=Z x$ and $p=\pi_{0}(Z)$ may be arbitrary functions. Recall that we have even infinitesimal symmetry of $\Omega$ if and only if the requirement (100) is satisfied. However clearly

$$
\begin{aligned}
d \pi_{0}= & d x \wedge \pi_{1} \\
& +G^{\prime \prime} \alpha_{1} \wedge \alpha_{2}+\left(G^{\prime \prime \prime} u_{3} \alpha_{2}+G^{\prime \prime} \alpha_{3}\right) \wedge \alpha_{0}
\end{aligned}
$$

and one can obtain the resolving equations as follows. First of all, we obtain equations

$$
\begin{gathered}
z D^{2} G^{\prime}+G^{\prime \prime} u_{3} a_{2}+G^{\prime \prime} a_{3}+p_{u_{0}}=\lambda D G^{\prime}, \\
p_{v_{0}}=\lambda \\
\left(a_{r}=\alpha_{r}(Z)\right)
\end{gathered}
$$

which determine coefficients $z$ and $\lambda$ analogously to (107). Moreover

$$
\begin{aligned}
G^{\prime \prime} & a_{2}-p_{u_{1}}-G^{\prime} p_{v_{0}} \\
& =G^{\prime \prime} a_{1}-G^{\prime \prime \prime} u_{3} a_{0}+p_{u_{2}} \\
& =G^{\prime \prime} a_{0}-p_{u_{3}}=p_{u_{r}}=0 \quad(r>3)
\end{aligned}
$$

are conditions for the unknown function $p=p\left(x, u_{0}, \ldots\right.$, $u_{3}, v_{0}$ ) analogous to (108). The "vertical" coefficients $a_{r}$ can be expressed in terms of functions $p, D p, D^{2} p$ and $D^{3} p$, by using the equation

$$
D^{2} G^{\prime} \cdot a_{0}=D^{2} G^{\prime} \cdot \alpha_{0}(Z)=\pi_{1}(Z)=D \pi_{0}(Z)=D p
$$

and the recurrence $a_{r+1}=D a_{r}$. As yet the calculations are much easier then for the above case of formulae (110); however, the resulting resolving system of three equations analogous to (112)-(114) is again complicated and will not 
be explicitly stated here. Remarkable task appears when we investigate the corresponding crucial requirements and try to determine the structure of function $p$ in terms of new coordinates. For instance, the "very prominent" and seemingly rather artificial case $(\kappa \iota)$ turns into the "simplest possible" and quite natural equation $d v / d x=\left(d^{2} u / d x^{2}\right)^{2}$ in new coordinates.

\section{Brief Digression to the Calculus of Variations}

The classical Lagrange problem of the calculus of variations deals with an underdetermined system of differential equations (better with a diffiety) together with a variational integral. We are interested in internal symmetries of this variational problem.

Let us start with a diffiety $\Omega \subset \Phi(\mathbf{M})$. We choose a standard filtration $\bar{\Omega}_{*}$ and the corresponding standard basis $\pi_{r}^{j}(j=1, \ldots, \mu(\Omega) ; r=0,1, \ldots)$. For better clarity, we suppose the controllable case $\mathscr{R}(\Omega)=0$. Let $x \in \mathscr{F}(\mathbf{M})$ be an independent variable. Let us consider $x$-parametrized solutions $\mathbf{p}$ of diffiety $\Omega$ in the sense

$$
\begin{gathered}
\mathbf{p}: \mathbf{I} \longrightarrow \mathbf{M} \quad(\mathbf{I} \subset \mathbb{R}), \\
\mathbf{p}^{*} \omega=0 \quad(\omega \in \Omega), \\
\mathbf{p}^{*} x=x \in \mathbf{I} \subset \mathbb{R} .
\end{gathered}
$$

Here $\mathbf{I} \subset \mathbb{R}$ is a closed interval $a \leq x \leq b$ with a little confusion: letter $x$ denotes both a function on $\mathbf{M}$ and the common coordinate (that is, a point) in $\mathbb{R}$.

Definition 33. A vector field $V \in \mathscr{T}(\mathbf{M})$ is called a variation of solution $\mathbf{p}$ of diffiety $\Omega$ if $\mathbf{p}^{*} \mathscr{L}_{V} \omega=0(\omega \in \Omega)$. This is a mere slight adaptation of the familiar classical concept.

Lemma 34. A vector field $V \in \mathscr{T}(\mathbf{M})$ is a variation of $\mathbf{p}$ if and only if

$$
\begin{array}{r}
\mathbf{p}^{*} \pi_{r+1}^{j}(V)=\mathbf{p}^{*} D \pi_{r}^{j}(V)=\frac{d}{d x} \mathbf{p}^{*} \pi_{r}^{j}(V) \\
(j=1, \ldots, \mu(\Omega) ; r=0,1, \ldots) .
\end{array}
$$

Proof. A variation $V$ satisfies $\mathbf{p}^{*} \mathscr{L}_{V} \pi_{r}^{j}=0$, where

$$
\begin{aligned}
\mathbf{p}^{*} \mathscr{L}_{V} \pi_{r}^{j} & \left.=\mathbf{p}^{*}(V\rfloor d \pi_{r}^{j}+d \pi_{r}^{j}(V)\right) \\
& =\mathbf{p}^{*}\left(-\pi_{r+1}^{j}(V)+D \pi_{r}^{j}(V)\right) d x
\end{aligned}
$$

by virtue of (55).

Remark 35. It follows easily that a vector field $Z \in \mathscr{T}(\mathbf{M})$ is a variation of diffiety $\Omega$ in the sense of Definition 8 if and only if $Z$ is a variation of every solution $\mathbf{p}$ of $\Omega$; see Lemma 23. Conversely, if $V$ is a variation of a solution $\mathbf{p}$ then there exist many variations $Z$ of $\Omega$ such that $Z=V$ at every point of $\mathbf{p}$, and they are characterized by the identities $\mathbf{p}^{*} \pi_{0}^{j}(Z)=\mathbf{p}^{*} \pi_{0}^{j}(V)(j=1, \ldots, \mu(\Omega))$ along the curve $\mathbf{p}$; see formula (72). We conclude that the concepts "variation $Z$ of $\Omega$ " and "variations $V$ of $\mathbf{p}$ " are closely related. Roughly saying, variations $V$ of $\mathbf{p}$ are "restrictions" of variations $Z$ of $\Omega$ to the curve $\mathbf{p}$.

Definition 36. A couple $\{\Omega, \varphi\}$ where $\Omega \subset \Phi(\mathbf{M})$ is a diffiety and $\varphi \in \Phi(\mathbf{M})$ is a differential form will be identified with a variational problem in the (common) sense that diffiety $\Omega$ represents the differential constraints to the variational integral $\int \varphi$. A solution $\mathbf{p}$ of $\Omega$ is called an extremal of this variational problem, if

$$
\left.\int \mathbf{p}^{*} \mathscr{L}_{V} \varphi=\int \mathbf{p}^{*} V\right\rfloor d \varphi=0 \quad(\text { special variations } V)
$$

for every variation $V$ of $\mathbf{p}$ which is vanishing at the endpoints $\mathbf{p}(a), \mathbf{p}(b) \in \mathbf{M}$. This definition provides the common classical extremals; see Remark 43.

Remark 37. The phrase "variation $V$ of $\mathbf{p}$ " can be replaced with "variation $V$ of $\Omega$ ". The form $\varphi$ can be replaced with arbitrary form $\varphi+\omega(\omega \in \Omega)$. The extremals do not change.

Theorem 38. To every standard basis of $\Omega$ and given $\varphi \in$ $\Phi(\mathbf{M})$ there exists unique form $\breve{\varphi} \in \Phi(\mathbf{M})$ such that

$$
\begin{gathered}
\breve{\varphi} \cong \varphi \quad(\bmod \Omega) \\
d \breve{\varphi} \cong 0 \quad\left(\bmod \Omega \wedge \Omega \text { and all initial forms } \pi_{0}^{j}\right)
\end{gathered}
$$

In accordance with (198) we assume that

$$
d \breve{\varphi} \cong \sum e^{j} \pi_{0}^{j} \wedge d x \quad(\bmod \Omega \wedge \Omega) .
$$

Then a solution $\mathbf{p}$ of $\Omega$ is extremal if and only if $\mathbf{p}^{*} e^{j}=0(j=$ $1, \ldots, \mu(\Omega))$ and therefore if and only if

$$
\left.\mathbf{p}^{*} Z\right\rfloor d \breve{\varphi}=0 \quad(Z \in \mathscr{T}(\mathbf{M}))
$$

for all vector fields $Z \in \mathscr{T}(\mathbf{M})$.

Proof (see [9]). For a given $\varphi \in \Phi(\mathbf{M})$, let us look at a toporder summand

$$
\begin{aligned}
d \varphi \cong \sum a_{r}^{j} \pi_{r}^{j} \wedge d x= & \cdots+a_{R}^{J} \pi_{R}^{J} \wedge d x \\
& (\bmod \Omega \wedge \Omega) .
\end{aligned}
$$

If $R>0$, the summand can be deleted if the primary differential form $\varphi$ is replaced with the new form $\varphi+a_{R}^{J} \pi_{R-1}^{J}$. The extremals do not change. The procedure is unique and terminates in form $\breve{\varphi}$ satisfying (198). Then (200) follows from the identity

$$
\left.\left.\mathbf{p}^{*} Z\right\rfloor d \breve{\varphi}=\mathbf{p}^{*} Z\right\rfloor \sum e^{j} \pi_{0}^{j} \wedge d x=\mathbf{p}^{*} \sum e^{j} \pi_{0}^{j}(Z) d x,
$$

where the functions $\pi_{0}^{j}(Z)$ may be quite arbitrary if $Z$ is a variation, see Lemma 34 . 
Definition 39. The differential form $\breve{\varphi}$ can be regarded for the internal Poincaré-Cartan form of our variational problem and equations $e^{j}=0(j=1, \ldots, \mu(\Omega))$ for the Euler-Lagrange system.

We turn to the symmetries.

Definition 40. A symmetry $\mathbf{m}$ of diffiety $\Omega$ is called a symmetry of variational problem $\{\Omega, \varphi\}$, if $\mathbf{m}^{*} \varphi \cong \varphi(\bmod \Omega)$. A variation (infinitesimal symmetry) $Z$ of $\Omega$ is called a variation (infinitesimal symmetry, resp.) of variational problem $\{\Omega, \varphi\}$, if $\mathscr{L}_{Z} \varphi \in \Omega$. Let $V \in \mathscr{T}(\mathbf{M})$ be a variation of a solution $\mathbf{p}$ of diffiety $\Omega$. Then $V$ is called a Jacobi vector field of $\mathbf{p}$, if moreover $\mathbf{p}^{*} \mathscr{L}_{V} \varphi=0$. Roughly saying, variations $Z$ of variational problem $\{\Omega, \varphi\}$ are "universal" Jacobi vector fields for all solutions $\mathbf{p}$ of $\Omega$. In classical theory, Jacobi vector fields are introduced only for the particular case when $\mathbf{p}$ is an extremal.

We will see in the following example that PoincaréCartan forms $\breve{\varphi}$ simplify the calculation of symmetries and variations. On this occasion, we also recall the following admirable result.

Theorem 41 (E. Noether). If $Z$ is a variation of variational problem $\{\Omega, \varphi\}$ and $\breve{\varphi}$ is a Poincaré-Cartan form then $\mathbf{p}^{*} \breve{\varphi}(Z)=$ const. for every extremal $\mathbf{p}$.

Proof. We have $\mathscr{L}_{Z} \breve{\varphi} \in \Omega, \mathbf{p}^{*} \omega=0(\omega \in \Omega)$, and therefore

$$
\begin{aligned}
0 & \left.=\mathbf{p}^{*} \mathscr{L}_{Z} \breve{\varphi}=\mathbf{p}^{*}(Z\rfloor d \breve{\varphi}+d \breve{\varphi}(Z)\right) \\
& =\mathbf{p}^{*} d \breve{\varphi}(Z)=d \mathbf{p}^{*} \breve{\varphi}(Z)
\end{aligned}
$$

by virtue of (200).

Remark 42. Many concepts of the classical calculus of variations lose the geometrical meaning if the higher-order symmetries are accepted; for example, this concerns the common concept of a nondegenerate variational problem and even the order of a variational integral. On the other hand, the most important concepts can be appropriately modified; for example, the Hilbert-Weierstrass extremality theory together with the Hamilton-Jacobi equations [18-21] since the Poincaré-Cartan forms $\breve{\varphi}$ make "absolute sense" along the extremals.

Remark 43. In the common classical calculus of variations, extremals $\mathbf{p}$ are defined by the property $\int \mathbf{p}^{*} \mathscr{L}_{V} \varphi=0$, where variations $V$ satisfy certain weak boundary conditions at the endpoints ("fixed ends" or transversality) in order to delete some "boundary effects" of the variational integral. Much stronger conditions appear in Definition 36. Therefore

classical extremals $\subset$ our extremals.

However, $\varphi$ can be replaced by the form $\breve{\varphi}$. Then

$$
\left.\int \mathbf{p}^{*} \mathscr{L}_{V} \breve{\varphi}=\int \mathbf{p}^{*} V\right\rfloor d \breve{\varphi}+\text { boundary term. }
$$

For the above special variations $V$, the boundary term vanishes. If $\mathbf{p}$ is extremal in the sense of Definition 36, then (200) and Remark 15 may be applied and it follows that

classical extremals つ our extremals.

In topical Griffiths' theory [22], extremals are defined by the property

$$
\left.\mathbf{p}^{*} Z\right\rfloor d(\varphi+\omega)=0
$$

(all $Z \in \mathscr{T}(\mathbf{M})$, appropriate $\omega \in \Omega$ depending on $\mathbf{p}$ )

which is clearly equivalent to the condition

$$
\left.\int \mathbf{p}^{*} Z\right\rfloor d(\varphi+\omega)=\int \mathbf{p}^{*} \mathscr{L}_{Z}(\varphi+\omega)=0
$$

(special vector fields $Z$, appropriate $\omega \in \Omega$ ),

where $Z$ are vector fields vanishing at the endpoints. This condition trivially implies

$$
\int \mathbf{p}^{*} \mathscr{L}_{V}(\varphi+\omega)=0 \text { hence } \int \mathbf{p}^{*} \mathscr{L}_{V} \varphi=0
$$

(special variations $V$ )

with variations $V$ vanishing at the endpoints; see Remark 35. Therefore

$$
\text { Griffiths extremals } \subset \text { our extremals. }
$$

The converse inclusion

$$
\text { Griffiths extremals つ our extremals }
$$

is, however, trivial since the universal form $\breve{\varphi}=\varphi+\breve{\omega}(\breve{\omega} \in \Omega)$ satisfies $\left.\mathbf{p}^{*} Z\right\rfloor d \breve{\varphi}=0$ even for every extremal in the sense of Definition 36. We conclude that all the mentioned concepts of extremals are identical. (We apologize for this hasty exposition. Roughly saying, the Griffiths' theory and our approach are almost identical. The Griffiths' correction $\omega \in$ $\Omega$ depending on $\mathbf{p}$ is made universal here. The classical approach rests on a special choice of boundary conditions for the variations $V$. However, such a special choice is misleading since it does not affect the resulting family of extremals and we prefer a universal choice here as well.)

\section{Particular Example of a Variational Integral}

A simple illustrative example is necessary at this place. Let us again deal with diffiety $\Omega$ of Section 6 . So we recall coordinates $x, u_{0}, u_{1}, v_{0}, v_{1}, \ldots$ of the underlying space $\mathbf{M}$, the contact forms $\alpha_{r}, \beta_{r}(r=0,1, \ldots)$ generating $\Omega$, the vector field

$$
\begin{aligned}
D & =\frac{\partial}{\partial x}+u_{1} \frac{\partial}{\partial u_{0}}+F \frac{\partial}{\partial u_{1}}+\sum v_{r+1} \frac{\partial}{\partial v_{r}} \\
& =\frac{\partial}{\partial x}+\sum_{\omega \in \Omega} 0 \cdot \frac{\partial}{\partial \omega} \in \mathscr{H},
\end{aligned}
$$


and the standard basis $\pi_{0}, \pi_{1}, \ldots$ of $\Omega$. We moreover introduce variational integrals

$$
\int \varphi \quad(\varphi=g d x, g \in \mathscr{F}(\mathbf{M})) .
$$

Assuming $\partial g / \partial \pi_{r}=0(r>R)$ and therefore

$$
\begin{aligned}
d g & =D g d x+\frac{\partial g}{\partial u_{0}} \alpha_{0}+\frac{\partial g}{\partial u_{1}} \alpha_{1}+\sum \frac{\partial g}{\partial v_{r}} \beta_{r} \\
& =D g d x+\frac{\partial g}{\partial \pi_{0}} \pi_{0}+\cdots+\frac{\partial g}{\partial \pi_{R}} \pi_{R},
\end{aligned}
$$

we introduce the functions

$$
\begin{gathered}
g_{R}=\frac{\partial g}{\partial \pi_{R}} \\
g_{r-1}=\frac{\partial g}{\partial \pi_{r-1}}-D g_{r} \quad(r=R, \ldots, 1) .
\end{gathered}
$$

Then

$$
\breve{\varphi}=g d x+g_{1} \pi_{0}+\cdots+g_{R} \pi_{R-1}
$$

is the Poincaré-Cartan form since the identity

$$
d \breve{\varphi}=g_{0} \cdot \pi_{0} \wedge d x \quad(\bmod \Omega \wedge \Omega)
$$

can be directly verified. In accordance with formula (199) where $e=e^{1}, \pi_{0}=\pi_{0}^{1}$ is abbreviated, we have $e=g_{0}$. Let us denote

$$
\begin{aligned}
e & =e[g]=g_{0} \\
& =\frac{\partial g}{\partial \pi_{0}}-D \frac{\partial g}{\partial \pi_{1}}+\cdots+(-1)^{R} D^{R} \frac{\partial g}{\partial \pi_{R}},
\end{aligned}
$$

for better clarity. The following simple result will be needed.

Lemma 44. Identity $e[g]=0$ is equivalent to the equation $g=D G$ with appropriate $G \in \mathscr{F}(\mathbf{M})$.

Proof. By virtue of (200), the identity is equivalent to the congruence $d \breve{\varphi} \cong 0(\bmod \Omega \wedge \Omega)$. However, if the rule $d(d \breve{\varphi})=0$ is applied to the congruence, it follows easily that $d \breve{\varphi}=0$ identically. Therefore $\breve{\varphi}=d G \cong D G d x(\bmod \Omega)$ by using the Poincaré lemma.

Let us mention symmetries $\mathbf{m}$ and variations $Z$ of our variational problem in more detail. In the favourable case $\mu(\Omega)=1$, the task is not difficult.

The symmetry $\mathbf{m}$ of our variational problem $\{\Omega, \varphi\}$ clearly preserves the unique Poincaré-Cartan form $\breve{\varphi}$ and therefore also the vector field $\mathscr{D}=D / g \in \mathscr{H}$ determined by the condition $\breve{\varphi}(\mathscr{D})=1$. We suppose $g \neq 0$ here. It follows that all differential forms

$$
\begin{gathered}
\left.\breve{\pi}_{0}=\mathscr{L}_{\mathscr{D}} \breve{\varphi}=\mathscr{D}\right\rfloor d \breve{\varphi}+d \breve{\varphi}(\mathscr{D})=e \pi_{0} \cdot \mathscr{D} x=\frac{e}{g} \pi_{0}, \\
\breve{\pi}_{r+1}=\mathscr{L}_{\mathscr{D}} \breve{\pi}_{r} \quad(r=0,1, \ldots)
\end{gathered}
$$

are preserved, too. Let us moreover suppose $e=e[g] \neq 0$. Clearly

$$
\begin{aligned}
\breve{\pi}_{1}=\frac{1}{g} \mathscr{L}_{D} \pi_{0}= & \frac{1}{g}\left(D \frac{e}{g} \pi_{0}+\frac{e}{g} \pi_{1}\right), \\
\breve{\pi}_{r+1} & =\frac{1}{g} \mathscr{L}_{\mathscr{D}} \pi_{r} \cong \frac{e}{g^{r+1}} \pi_{r} \\
& \left(\bmod d x, \pi_{0}, \ldots, \pi_{r}\right) .
\end{aligned}
$$

Therefore $\breve{\varphi}, \breve{\pi}_{0}, \breve{\pi}_{1}, \ldots$ is invariant basis of module $\Phi(\mathbf{M})$ in the sense

$$
\begin{gathered}
\mathbf{m}^{*} \breve{\varphi}=\breve{\varphi}, \\
\mathbf{m}^{*} \breve{\pi}_{r}=\breve{\pi}_{r} \quad(r=0,1, \ldots) .
\end{gathered}
$$

It follows that the symmetries $\mathbf{m}$ of our variational problem $\{\Omega, \varphi\}$ can be comfortably determined. Quite analogous conclusion can be made for the infinitesimal symmetries, of course.

Passing to the variations $Z$ of the variational problem, we have explicit formula (99) for the variations of $\Omega$ and moreover condition $\mathscr{L}_{Z} \varphi \in \Omega$ equivalent to $\mathscr{L}_{Z} \breve{\varphi} \in \Omega$. However

$$
\begin{aligned}
\mathscr{L}_{Z} \breve{\varphi} & =Z\rfloor d \breve{\varphi}+d \breve{\varphi}(Z) \\
& \cong Z\rfloor\left(e \pi_{0} \wedge d x\right)+D \breve{\varphi}(Z) d x \\
& =(e p+D \breve{\varphi}(Z)) d x \quad(\bmod \Omega)
\end{aligned}
$$

and therefore

$$
\begin{aligned}
& 0=e p+D \breve{\varphi}(Z)=e p+D G \\
& \left(G=g z+g_{0} p+g_{1} D p+\cdots+g_{R} D^{R} p\right) .
\end{aligned}
$$

Assume $g \neq 0$. We obtain condition $e[e p]=0$ for the unknown function $p$. In more precise notation and in full detail

$$
\begin{aligned}
e & {[e[g] p] } \\
& =\left(\frac{\partial}{\partial \pi_{0}}-D \frac{\partial}{\partial \pi_{1}}+\cdots\right)\left(\frac{\partial g}{\partial \pi_{0}}-D \frac{\partial g}{\partial \pi_{1}}+\cdots\right) p \\
& =0 .
\end{aligned}
$$

This is formally a very simple condition concerning the unknown function $p$; alas, it is not easy to be resolved. Paradoxically, variations $Z$ cause serious difficulties.

For better clarity, we continue this example with particular choice of the variational integral. Let us consider variational integral $\int g\left(x, u_{0}, v_{0}\right) d x$. Equation (214) reads

$$
\begin{aligned}
d g & =D g d x+\frac{\partial g}{\partial u_{0}} \alpha_{0}+\frac{\partial g}{\partial v_{0}} \beta_{0} \\
& =D g d x+\frac{\partial g}{\partial \pi_{0}} \pi_{0}+\frac{\partial g}{\partial \pi_{1}} \pi_{1}+\frac{\partial g}{\partial \pi_{2}} \pi_{2}
\end{aligned}
$$


and it follows that

$$
\begin{gathered}
\frac{\partial g}{\partial \pi_{0}}=-2 g_{u_{0}} \frac{D f}{C}+g_{v_{0}} \frac{A}{C^{2}}, \\
\frac{\partial g}{\partial \pi_{1}}=g_{u_{0}} \frac{f}{C}+g_{v_{0}} \frac{B}{C^{2}}, \quad \frac{\partial g}{\partial \pi_{2}}=\frac{g_{v_{0}}}{C}
\end{gathered}
$$

by using (93). We have $R=2$ and therefore

$$
\begin{gathered}
\breve{\varphi}=g d x+\left(\frac{\partial g}{\partial \pi_{1}}-D \frac{\partial g}{\partial \pi_{2}}\right) \pi_{0}+\frac{\partial g}{\partial \pi_{2}} \pi_{1}, \\
e=\frac{\partial g}{\partial \pi_{0}}-D \frac{\partial g}{\partial \pi_{1}}+D^{2} \frac{\partial g}{\partial \pi_{2}}
\end{gathered}
$$

by virtue of (215)-(218). Both the Poincaré-Cartan form $\breve{\varphi}$ and the Euler-Lagrange equation $e=0$ can be expressed in terms of common coordinates, if derivatives (226) are inserted. We omit the final formulae here. Passing to the symmetries $\mathbf{m}$, we may simulate the moving frames method and express the differential

$$
\begin{aligned}
d \breve{\varphi} & =\sum C_{r} \breve{\pi}_{r} \wedge \breve{\varphi}+\sum_{r<s} C_{r s} \breve{\pi}_{r} \wedge \breve{\pi}_{s} \\
& =\breve{\pi}_{0} \wedge \breve{\varphi}+\sum_{r<s} C_{r s} \breve{\pi}_{r} \wedge \breve{\pi}_{s}
\end{aligned}
$$

in terms of the invariant basis (221). Then all coefficients $C_{r s}$ are invariants of symmetry $\mathbf{m}$; that is,

$$
\mathbf{m}^{*} C_{r s}=C_{r s} \text { hence } \mathbf{m}^{*} \mathscr{D}^{k} C_{r s}=\mathscr{D}^{k} C_{r s} \quad\left(\mathscr{D}=\frac{D}{g}\right) .
$$

In fact we have obtained all invariants. (Hint: for instance, differential

$$
\begin{aligned}
d \breve{\pi}_{0}= & d \mathscr{L}_{\mathscr{D}} \breve{\varphi}=\mathscr{L}_{\mathscr{D}} d \breve{\varphi} \\
= & \sum \mathscr{D} C_{r s} \breve{\pi}_{r} \wedge \breve{\pi}_{s}+\mathscr{L}_{\mathscr{D}}\left(\breve{\pi}_{0} \wedge \breve{\varphi}\right) \\
& +\sum C_{r s} \mathscr{L}_{\mathscr{D}}\left(\breve{\pi}_{r} \wedge \breve{\pi}_{s}\right)
\end{aligned}
$$

does not provide any novelty.) It follows that the symmetry problem is resolved. Compatibility of the system of (229) ensures the existence of symmetries $\mathbf{m}$ of the variational problem $\{\Omega, \varphi\}$ since the Frobenius theorem can be applied to the Pfaffian system (221). In the most favourable case, $C_{r s}$ are even constants. Explicit calculation of invariants $C_{r s}$ is a lengthy but routine procedure. First of all

$$
\begin{aligned}
d \breve{\varphi} \cong & d g_{1} \wedge \pi_{0}+d g_{2} \wedge \pi_{1}+g_{1} d \pi_{0} \\
& +g_{2} d \pi_{1} \quad(\bmod d x)
\end{aligned}
$$

by using the primary formula (216). Then

$$
d g_{1} \cong \sum \frac{\partial g_{1}}{\partial \pi_{r}} \pi_{r}, \quad d g_{2} \cong \sum \frac{\partial g_{2}}{\partial \pi_{r}} \pi_{r} \quad(\bmod d x)
$$

may be substituted where the coefficient can be determined analogously as in (226). As the differential

$$
\begin{aligned}
d \pi_{0} \cong & \beta_{1} \wedge\left(f^{\prime} \alpha-f f^{\prime} \beta_{0}+f^{\prime \prime} v_{2} \alpha_{0}\right) \\
& +f^{\prime} \beta_{2} \wedge \alpha_{0} \quad(\bmod d x)
\end{aligned}
$$

is concerned, we refer to formula in Section 6. The contact forms must be replaced with the standard basis by using the right-hand formulae (93). Then we may use the lucky identity

$$
d \pi_{1}=d \mathscr{L}_{D} \pi_{0}=\mathscr{L}_{D} d \pi_{0}
$$

in order to determine the last summand in (231). In the end, the standard basis $\pi_{r}$ in (231) can be easily replaced by the invariant forms $\breve{\pi}_{r}(r=0,1, \ldots)$ and we are done.

\section{The Order-Increasing Case}

Let us eventually return to the main topic, the differential equations. We will finish this paper with decisive examples of higher-order symmetries, namely, with symmetries of the Monge equation

$$
\frac{d w}{d x}=F\left(x, u, v, w, \frac{d u}{d x}, \frac{d v}{d x}\right)
$$

involving three unknown functions $u=u(x), v=v(x)$, and $w=w(x)$. Let us directly turn to the internal theory carried out by using the underlying space $\mathbf{M}$ with coordinates

$$
x, u_{r}, v_{r}, w_{0} \quad(r=0,1, \ldots),
$$

diffiety $\Omega \subset \Phi(\mathbf{M})$ with the basis

$$
\begin{gathered}
\alpha_{r}=d u_{r}-u_{r+1} d x, \\
\beta_{r}=d v_{r}-v_{r+1} d x \quad(r=0,1, \ldots), \\
\gamma_{0}=d w_{0}-F\left(x, u_{0}, v_{0}, w_{0}, u_{1}, v_{1}\right) d x
\end{gathered}
$$

and the total derivative

$$
D=\frac{\partial}{\partial x}+\sum u_{r+1} \frac{\partial}{\partial u_{r}}+\sum v_{r+1} \frac{\partial}{\partial v_{r}}+F \frac{\partial}{\partial w_{0}} \in \mathscr{H} .
$$

We also introduce functions and differential forms

$$
\begin{aligned}
& w_{r}=D^{r} w_{0} \in \mathscr{F}(\mathbf{M}), \\
& \gamma_{r}=\mathscr{L}_{D}^{r} \gamma_{0}=d w_{r}-w_{r+1} d x \in \Omega \\
& \quad(r=0,1, \ldots)
\end{aligned}
$$

for the formal reasons. The natural filtration $\Omega_{*}$ in accordance with the order is such that the forms $\alpha_{0}, \ldots, \alpha_{l}, \beta_{0}, \ldots, \beta_{l}, \gamma_{0}$ are taken for the basis of submodule $\Omega_{l} \subset \Omega(l=0,1, \ldots)$. Let us determine the corresponding standard filtration $\bar{\Omega}_{*}$. Clearly

$$
\begin{gathered}
\mathscr{L}_{D} \gamma_{0}=\gamma_{1}=F_{u_{0}} \alpha_{0}+F_{v_{0}} \beta_{0}+F_{w_{0}} \gamma_{0} \\
+F_{u_{1}} \alpha_{1}+F_{v_{1}} \beta_{1}
\end{gathered}
$$


and therefore

$$
\begin{aligned}
& \mathscr{L}_{D}\left(\gamma_{0}-F_{u_{1}} \alpha_{0}-F_{v_{1}} \beta_{0}\right) \\
& =\left(F_{u_{0}}-D F_{u_{1}}\right) \alpha_{0}+\left(F_{v_{0}}-D F_{v_{1}}\right) \beta_{0}+F_{w_{0}} \gamma_{0} \in \Omega_{0} .
\end{aligned}
$$

Denoting $\pi=\gamma_{0}-F_{u_{1}} \alpha_{0}-F_{v_{1}} \beta_{0}$, we obtain

$$
\begin{gathered}
\mathscr{L}_{D} \pi=\left(F_{u_{0}}-D F_{u_{1}}\right) \alpha_{0}+\left(F_{v_{0}}-D F_{v_{1}}\right) \beta_{0}+F_{w_{0}} \gamma_{0} \\
=A \alpha_{0}+B \beta_{0}+F_{w_{0}} \pi \in \Omega_{0} \\
\left(A=F_{u_{0}}-D F_{u_{1}}+F_{w_{0}} F_{u_{1}}, B=F_{v_{0}}-D F_{v_{1}}+F_{w_{0}} F_{v_{1}}\right) .
\end{gathered}
$$

We will not deal with the case when $A=B=0$ identically. Let us instead suppose that $A \neq 0$ from now on. Then $\mathscr{R}(\Omega)=$ 0 and we may introduce standard filtration $\bar{\Omega}_{*}$ of diffiety $\Omega$ where the form

$$
\pi_{0}^{1}=\pi=\gamma_{0}-F_{u_{1}} \alpha_{0}-F_{v_{1}} \beta_{0}
$$

generates $\bar{\Omega}_{0}$ and in general the forms

$$
\begin{aligned}
& \pi_{r}^{1}=\mathscr{L}_{D}^{r} \pi \quad(r=0, \ldots, l), \\
& \pi_{r}^{2}=\beta_{r} \quad(r=0, \ldots, l-1)
\end{aligned}
$$

generate module $\bar{\Omega}_{l}(l \geq 1)$. Notation (53) with indices is retained here. With this preparation, we are passing to the symmetries of diffiety $\Omega$. Theorem 26 and Remark 27 fail since $\mu(\Omega)=2$ in our case. There exist many standard filtrations of $\Omega$ and we may also expect the existence of the order-destroying symmetries.

The preparation is done; however, before passing to quite explicit examples, certain general aspects are worth mentioning. We recall Figure 3 which can be transparently illustrated just at this place for the first time.

First of all, every order-preserving symmetry $\mathbf{m}$ on scheme (a) of Figure 3 obviously satisfies certain formulae

$$
\begin{gathered}
\mathbf{m}^{*} \alpha_{0}=a^{1} \alpha_{0}+a^{2} \beta_{0}+a^{3} \gamma_{0}, \\
\mathbf{m}^{*} \beta_{0}=b^{1} \alpha_{0}+b^{2} \beta_{0}+b^{3} \gamma_{0}, \\
\mathbf{m}^{*} \gamma_{0}=c^{1} \alpha_{0}+c^{2} \beta_{0}+c^{3} \gamma_{0}, \\
\operatorname{det}\left(\begin{array}{lll}
a^{1} & a^{2} & a^{3} \\
b^{1} & b^{2} & b^{3} \\
c^{1} & c^{2} & c^{3}
\end{array}\right) \neq 0,
\end{gathered}
$$

where the coefficients cannot be in fact arbitrary since they are subjected to identity (240). In more detail, we have

$$
\begin{aligned}
& D W \cdot \mathbf{m}^{*} \gamma_{1} \\
& \quad=\mathscr{L}_{D} \mathbf{m}^{*} \gamma_{0} \\
& \quad=D c^{1} \alpha_{0}+D c^{2} \beta_{0}+D c^{3} \gamma_{0}+c^{1} \alpha_{1}+c^{2} \beta_{1}+c^{3} \gamma_{1}
\end{aligned}
$$

in accordance with (63). Alternatively (240) implies

$$
\mathbf{m}^{*} \gamma_{1}=\mathbf{m}^{*} F_{u_{0}} \cdot \mathbf{m}^{*} \alpha_{0}+\cdots+\mathbf{m}^{*} F_{v_{1}} \cdot \mathbf{m}^{*} \beta_{1},
$$

where the forms $\mathbf{m}^{*} \alpha_{0}, \ldots, \mathbf{m}^{*} \beta_{1}$ can be expressed in terms of forms $\alpha_{0}, \ldots, \gamma_{1}$. The comparison provides many unpleasant interrelations among coefficients $a^{1}, \ldots, c^{3}$.

However, by using the standard basis, the same symmetry satisfies shorter formulae

$$
\mathbf{m}^{*} \pi_{0}^{1}=a \pi_{0}^{1}
$$

(hence, automatically $D W \mathbf{m}^{*} \pi_{1}^{1}=D a \pi_{0}^{1}+a \pi_{1}^{1}$ ),

$$
\mathbf{m}^{*} \pi_{0}^{2}=b_{0}^{1} \pi_{0}^{1}+b_{0}^{2} \pi_{0}^{2}+b_{1}^{1} \pi_{1}^{1}
$$

with coefficients subjected only to the inequalities $a \neq 0$ and $b_{0}^{2} \neq 0$ at this place. We employ the fact that both triples $\alpha_{0}, \beta_{0}, \gamma_{0}$ and $\pi_{0}^{1}, \pi_{0}^{2}, \pi_{1}^{1}$ are bases of module $\Omega_{0}$. Moreover $\mathbf{m}^{*}$ preserves the natural filtration $\Omega_{*}$ and therefore also the corresponding standard filtration $\bar{\Omega}_{*}$. Especially, the initial term $\bar{\Omega}_{0}$ is preserved and $\mathbf{m}^{*} \pi_{0}^{1}$ is a mere multiple of $\pi_{0}^{1}$.

The order-preserving infinitesimal symmetry $Z$ corresponding to scheme (a) satisfies either the system

$$
\begin{gathered}
\mathscr{L}_{Z} \alpha_{0}=\lambda_{1} \alpha_{0}+\lambda_{2} \beta_{0}+\lambda_{3} \gamma_{0} \\
\mathscr{L}_{Z} \beta_{0}=\mu_{1} \alpha_{0}+\mu_{2} \beta_{0}+\mu_{3} \gamma_{0} \\
\mathscr{L}_{Z} \gamma_{0}=\nu_{1} \alpha_{0}+\nu_{2} \beta_{0}+\nu_{3} \gamma_{0}
\end{gathered}
$$

with coefficients subjected to many identities analogous as above or, alternatively, the equivalent and shorter system

$$
\begin{aligned}
& \mathscr{L}_{Z} \pi_{0}^{1}=\mu \pi_{0}^{1} \\
& \text { (hence } \left.\mathscr{L}_{Z} \pi_{1}^{1}=D \mu \pi_{0}^{1}+(\mu-D Z x) \pi_{1}^{1}\right), \\
& \qquad \mathscr{L}_{Z} \pi_{0}^{2}=\lambda_{0}^{1} \pi_{0}^{1}+\lambda_{0}^{2} \pi_{0}^{2}+\lambda_{1}^{1} \pi_{1}^{1}
\end{aligned}
$$

with arbitrary coefficients in terms of the standard basis. For the middle equation use the identity

$$
\mathscr{L}_{Z} \pi_{1}^{1}=\mathscr{L}_{Z} \mathscr{L}_{D} \pi_{0}^{1}=\mathscr{L}_{D} \mathscr{L}_{Z} \pi_{0}^{1}-D z \pi_{1}^{1} \quad(z=Z x) .
$$

This follows from the Lie bracket formula $[D, Z]=D z \cdot D$ which is true if and only if $Z$ is a variation of diffiety $\Omega$. The Cartan's general equivalence method [23] can be applied to this order-preserving symmetry problem; however, we will mention the Lie approach later on.

With this result, the simplest possible order-increasing symmetry $\mathbf{m}$ on scheme (c) of Figure 3 can be introduced by the equations

$$
\begin{gathered}
\mathbf{m}^{*} \pi_{0}^{1}=a^{1} \pi_{0}^{1}+a^{2} \pi_{0}^{2}, \\
\mathbf{m}^{*} \pi_{0}^{2}=b^{1} \pi_{0}^{1}+b^{2} \pi_{0}^{2}+b\left(a^{1} \pi_{1}^{1}+a^{2} \pi_{1}^{2}\right), \\
\operatorname{det}\left(\begin{array}{ll}
a^{1} & b^{1}-b D a^{1} \\
a^{2} & b^{2}-b D a^{2}
\end{array}\right) \neq 0 .
\end{gathered}
$$


Let us prove the invertibility of $\mathbf{m}$. Clearly

$$
\begin{aligned}
D W \mathbf{m}^{*} \pi_{1}^{1} & =\mathscr{L}_{D} \mathbf{m}^{*} \pi_{0}^{1} \\
& =D a^{1} \pi_{0}^{1}+D a^{2} \pi_{0}^{2}+a^{1} \pi_{1}^{1}+a^{2} \pi_{1}^{2}
\end{aligned}
$$

and it follows that

$$
\begin{aligned}
\mathbf{m}^{*} & \pi_{0}^{2}-b D W \mathbf{m}^{*} \pi_{1}^{1} \\
& =\left(b^{1}-b D a^{1}\right) \pi_{0}^{1}+\left(b^{2}-b D a^{2}\right) \pi_{0}^{2} \in \mathbf{m}^{*} \Omega .
\end{aligned}
$$

Inclusions $\pi_{0}^{1}, \pi_{0}^{2} \in \mathbf{m}^{*} \Omega$ therefore hold true and Lemma 21 can be applied.

In order to state another example to scheme (c), let us consider the equations

$$
\begin{aligned}
& \mathbf{m}^{*} \pi_{0}^{1}=a_{0}^{1} \pi_{0}^{1}+a_{0}^{2} \pi_{0}^{2}+a_{1}^{1} \pi_{1}^{1}+a_{1}^{2} \pi_{1}^{2}, \\
& \mathbf{m}^{*} \pi_{0}^{2}=b_{0}^{1} \pi_{0}^{1}+b_{0}^{2} \pi_{0}^{2}+b_{1}^{1} \pi_{1}^{1}+b_{1}^{2} \pi_{1}^{2} .
\end{aligned}
$$

Invertibility of such morphism $\mathbf{m}$ is ensured if $b_{1}^{i}=b a_{1}^{i}, b_{0}^{i}-$ $b a_{0}^{i}=a a_{1}^{i}(i=1,2)$ for appropriate factors $a \neq 0, b$ and if moreover

$$
\operatorname{det}\left(\begin{array}{ll}
a_{1}^{1} & a_{0}^{1}-D a_{1}^{1} \\
a_{1}^{2} & a_{0}^{2}-D a_{1}^{2}
\end{array}\right) \neq 0 .
$$

For the proof of invertibility, apply $\mathscr{L}_{D}$ to the inclusion

$$
\frac{1}{a}\left(\mathbf{m}^{*} \pi_{0}^{2}-b \mathbf{m}^{*} \pi_{0}^{1}\right)=a_{1}^{1} \pi_{0}^{1}+a_{1}^{2} \pi_{0}^{2} \in \mathbf{m}^{*} \Omega
$$

and verify that $\pi_{0}^{1}, \pi_{0}^{2} \in \mathbf{m}^{*} \Omega$.

In both examples, the common general equivalence method [23] fails. The corresponding variations $Z$ can be introduced and are rather interesting though they do not generate any symmetry groups. See Remark 46 below.

It is also easy to illustrate scheme (b) of Figure 3 by using the symmetries $\mathbf{m}$ and infinitesimal symmetries $Z$ such that

$$
\begin{gathered}
\mathbf{m}^{*} \pi_{0}^{1}=a^{1} \pi_{0}^{1}+a^{2} \pi_{0}^{2}, \\
\mathbf{m}^{*} \pi_{0}^{2}=b^{1} \pi_{0}^{1}+b^{2} \pi_{0}^{2}, \\
\operatorname{det}\left(\begin{array}{ll}
a^{1} & a^{2} \\
b^{1} & b^{2}
\end{array}\right) \neq 0, \\
\mathscr{L}_{Z} \pi_{0}^{1}=\lambda^{1} \pi_{0}^{1}+\lambda^{2} \pi_{0}^{2}, \\
\mathscr{L}_{Z} \pi_{0}^{2}=\mu^{1} \pi_{0}^{1}+\mu^{2} \pi_{0}^{2} .
\end{gathered}
$$

(Hint: Theorem 24 can be trivially applied and the natural filtration is not preserved, if $a^{2} \neq 0$ and $\lambda^{2} \neq 0$.) Another example is provided by the equations

$$
\begin{gathered}
\mathbf{m}^{*} \pi_{0}^{1}=a_{0}^{1} \pi_{0}^{1}+a_{0}^{2} \pi_{0}^{2}+a_{1}^{2} \pi_{1}^{2}, \\
\mathbf{m}^{*} \pi_{0}^{2}=b \pi_{0}^{2}, \\
\mathscr{L}_{Z} \pi_{0}^{1}=\mu_{0}^{1} \pi_{0}^{1}+\mu_{0}^{2} \pi_{0}^{2}+\mu_{1}^{2} \pi_{1}^{2}, \\
\mathscr{L}_{Z} \pi_{0}^{2}=\mu \pi_{0}^{2}
\end{gathered}
$$

"symmetrical" to the order-preserving case. The classical Lie's infinitesimal symmetries and the Cartan's equivalence method can be both applied without any change.

We have briefly indicated only the simplest devices here and refer to [2, Section 4] for the universal construction. A complete overview of all possible higher-order symmetries of (235) is lying beyond any actual imagination. For instance, the composition $\mathbf{m}_{1} \circ \mathbf{m}_{2}$ of symmetries and the conjugate groups $\mathbf{m} \circ \mathbf{m}(\lambda) \circ \mathbf{m}^{-1}$ to a given group provide much more complicated examples than the original components $\mathbf{m}_{1}, \mathbf{m}_{2}, \mathbf{m}$, and $\mathbf{m}(\lambda)$. The definition equations for such composition of symmetries can be directly found and they look rather depressively for the time being.

\section{Concluding Examples on Infinitesimal Symmetries}

We deal only with a simplified equation (235), namely, with the equation

$$
\frac{d w}{d x}=F\left(\frac{d u}{d x}, \frac{d v}{d x}\right)
$$

for good reasons to be clarified in the Appendix. Let us abbreviate

$$
\begin{gathered}
F_{1}=F_{u_{1}}, \quad F^{1}=F_{v_{1}}, \quad F_{11}=F_{u_{1} u_{1}}, \\
F_{1}^{1}=F_{u_{1} v_{1}}, \quad F^{11}=F_{v_{1} v_{1}}
\end{gathered}
$$

from now on. The crucial identity (240) then reads

$$
\mathscr{L}_{D} \gamma_{0}=\gamma_{1}=F_{1} \alpha_{1}+F^{1} \beta_{1}
$$

and we recall the standard basis $\pi_{r}^{1}=\mathscr{L}_{D}^{r} \pi_{0}^{1}, \pi_{r}^{2}=\mathscr{L}_{D}^{r} \pi_{0}^{2}=$ $\beta_{r}(r=0,1, \ldots)$, where

$$
\begin{aligned}
& \pi_{0}^{1}=\gamma_{0}-F_{1} \alpha_{0}-F^{1} \beta_{0}, \\
& \pi_{1}^{1}=-D F_{1} \alpha_{0}-D F^{1} \beta_{0}
\end{aligned}
$$

in terms of the simplified notation. The formulae

$$
\begin{gathered}
d \pi_{0}^{1}=d x \wedge \pi_{1}^{1}+\left(F_{11} \alpha_{0}+F_{1}^{1} \beta_{0}\right) \wedge \alpha_{1} \\
+\left(F_{1}^{1} \alpha_{0}+F^{11} \beta_{0}\right) \wedge \beta_{1}, \\
d \pi_{0}^{2}=d x \wedge \beta_{1}
\end{gathered}
$$

easily follow. On this occasion, we also recall more general adjustments

$$
\begin{gathered}
\lambda \mathbf{m}^{*} \mathscr{L}_{D} \omega=\mathscr{L}_{D} \mathbf{m}^{*} \omega \\
\left(\omega \in \Omega, \lambda=D W, W=\mathbf{m}^{*} x\right), \\
\left(\mathscr{L}_{D} \omega\right)(Z)=D(\omega(Z)) \quad(\omega \in \Omega)
\end{gathered}
$$

of Lemmas 17 and 23. The factor $\lambda \neq 0$ appearing here can be defined by the congruence $\mathbf{m}^{*} d x \cong \lambda d x(\bmod \Omega)$ as well. 
Several symmetry problems for (260) will be mentioned. We start with examples on infinitesimal symmetries $Z$ and demonstrate our approach both using the traditional orderpreserving case and then employing two technically quite analogous order-increasing symmetry problems. The calculations are elementary but not of a mere mechanical nature and the concise form of the final results is worth attention. That is, by using the series (268) with the standard basis, the unknown functions $z, p$, and $q$ satisfy quite reasonable and explicitly solvable conditions. Denoting

$$
\begin{gathered}
a_{0}=\alpha_{0}(Z), \quad a_{1}=\alpha_{1}(Z)=D a_{0}, \\
p=\pi_{0}^{1}(Z), \quad q=\pi_{0}^{2}(Z)=\beta_{0}(Z), \\
z=Z x,
\end{gathered}
$$

we simulate the procedure of Section 6 and our method again rests on the explicit formula

$$
Z=z \frac{\partial}{\partial x}+\sum D^{r} p \frac{\partial}{\partial \pi_{r}^{1}}+\sum D^{r} q \frac{\partial}{\partial \pi_{r}^{2}}
$$

for all variations $Z$. We recall that infinitesimal symmetries $Z$ moreover satisfy certain additional requirements in order to ensure the conditions of Theorem 24. The choice of such requirements which is arbitrary to a large extent (dotted lines in Figure 3(b)) strongly affects the final result, the resulting symmetries $Z$. Altogether taken, reasonings of this Section 10 belong to the Lie's theory appropriately adapted to the infinitedimensional spaces. On the contrary, we will conclude this paper with only few remarks on the true (not group-like) higher-order symmetries $\mathbf{m}$ in subsequent Section 11. The reasonings can be related to the E. Cartan's general equivalence method $[16,23]$ and they would deserve more space than it is possible here.

Let us turn to proper examples.

(ı) The Order-Preserving Symmetry Problem. We again intentionally start with a mere "traditional" case. Let us deal with infinitesimal symmetries $Z$ satisfying

$$
\begin{gathered}
\mathscr{L}_{Z} \pi_{0}^{1}=\mu \pi_{0}^{1}=\mu\left(\gamma_{0}-F_{1} \alpha_{0}-F^{1} \beta_{0}\right), \\
\mathscr{L}_{Z} \pi_{0}^{2}=\lambda_{0}^{1} \pi_{0}^{1}+\lambda_{0}^{2} \pi_{0}^{2}+\lambda_{1}^{1} \pi_{1}^{1} \\
=\mu^{1} \alpha_{0}+\mu^{2} \beta_{0}+\mu^{3} \gamma_{0} .
\end{gathered}
$$

We use the "hybrid" equations involving both the standard basis and the contact forms. Let us recall the explicit formula (268) for all variations. We have moreover the above equations (269) in order to obtain the true infinitesimal symmetries. In more detail

$$
\begin{gathered}
Z\rfloor d \pi_{0}^{1}+d p=\mu\left(\gamma_{0}-F_{1} \alpha_{0}-F^{1} \beta_{0}\right), \\
Z\rfloor d \pi_{0}^{2}+d q=\mu^{1} \alpha_{0}+\mu^{2} \beta_{0}+\mu^{3} \gamma_{0}
\end{gathered}
$$

should be satisfied. Analogously as in Section 6, this is expressed by the resolving system

$$
\begin{gathered}
z D F_{1}+F_{11} a_{1}+F_{1}^{1} D q=p_{u_{0}}+\mu F_{1}, \\
z D F^{1}+F_{1}^{1} a_{1}+F^{11} D q=p_{v_{0}}+\mu F^{1}, \\
F_{11} a_{0}+F_{1}^{1} q+p_{u_{1}}=0, \\
F_{1}^{1} a_{0}+F^{11} q+p_{v_{1}}=0, \\
p_{w_{0}}=\mu, \\
p_{u_{r}}=p_{v_{r}}=0 \quad(r \geq 2), \\
q_{u_{0}}=\mu^{1}, \quad q_{w_{0}}=\mu^{3}, \\
q_{u_{r}}=0 \quad(r \geq 1), \\
z+q_{v_{1}}=0, \\
q_{v_{r}}=0 \quad(r \geq 2)
\end{gathered}
$$

by using (264) and $\beta_{1}(Z)=D \beta_{0}(Z)=D q$. It follows that only (271) with $\mu=p_{w_{0}}, z=-q_{v_{1}}$ inserted and coefficients $a_{0}, a_{1}$ given by

$$
a_{0} D F_{1}+q D F^{1}+D p=0, \quad a_{1}=D a_{0}
$$

are the most important.

Let us denote $\Delta=\left(F_{1}^{1}\right)^{2}-F_{11} F^{11}$ and assume $\Delta \neq 0$ from now on. Equations (271) are equivalent to

$$
\begin{aligned}
& \Delta D q=\operatorname{det}\left(\begin{array}{cc}
p_{u_{0}}+p_{w_{0}} F_{1}+q_{v_{1}} D F_{1} & F_{11} \\
p_{v_{0}}+p_{w_{0}} F^{1}+q_{v_{1}} D F^{1} & F_{1}^{1}
\end{array}\right), \\
& \Delta a_{1}=\operatorname{det}\left(\begin{array}{cc}
F_{1}^{1} & p_{u_{0}}+p_{w_{0}} F_{1}+q_{v_{1}} D F_{1} \\
F^{11} & p_{v_{0}}+p_{w_{0}} F^{1}+q_{v_{1}} D F^{1},
\end{array}\right), \\
& \Delta q=\operatorname{det}\left(\begin{array}{cc}
F_{11} & p_{u_{1}} \\
F_{1}^{1} & p_{v_{1}}
\end{array}\right), \\
& \Delta a_{0}=\operatorname{det}\left(\begin{array}{cc}
p_{u_{1}} & F_{1}^{1} \\
p_{v_{1}} & F^{11}
\end{array}\right) .
\end{aligned}
$$

We have unknown functions

$$
\begin{gathered}
p=p\left(x, u_{0}, v_{0}, w_{0}, u_{1}, v_{1}\right), \\
q=q\left(x, u_{0}, v_{0}, w_{0}, v_{1}\right)
\end{gathered}
$$

and let us pass to the solution of (273), (274), and (275).

The first equation (273) multiplied by function $\Delta$ reads

$$
\begin{aligned}
& \left(F_{11} u_{2}+F_{1}^{1} v_{2}\right) \Delta a_{0}+\left(F_{1}^{1} u_{2}+F^{11} v_{2}\right) \Delta q \\
& +\left(\mathscr{D} p+p_{u_{1}} u_{2}+p_{v_{1}} v_{2}\right) \Delta=0
\end{aligned}
$$

and therefore implies only the identity

$$
\mathscr{D} p=0 \quad\left(\mathscr{D}=\frac{\partial}{\partial x}+u_{1} \frac{\partial}{\partial u_{0}}+v_{1} \frac{\partial}{\partial v_{0}}+F \frac{\partial}{\partial w_{0}}\right),
$$


if both equations (275) are accepted (direct verification). Alternatively saying, second equation (275) can be regarded for a definition of function $a_{0}$ if (278) is taken into account. Let us denote $G=\Delta a_{0}$ for a moment. Then

$$
\begin{gathered}
D G=D \Delta \cdot a_{0}+\Delta \cdot D a_{0}=D \Delta \cdot \frac{G}{\Delta}+\Delta \cdot a_{1}, \\
\Delta \cdot D G-G \cdot D \Delta=a_{1} \cdot(\Delta)^{2}
\end{gathered}
$$

and the second equation (274) reads

$$
\Delta \cdot D G-G \cdot D \Delta=\Delta \cdot \operatorname{det}(\cdots)
$$

with the same determinant. Lower-order terms clearly provide the equation

$$
\mathscr{D} G=\operatorname{det}\left(\begin{array}{cc}
F_{1}^{1} & p_{u_{0}}+p_{w_{0}} F_{1} \\
F^{11} & p_{v_{0}}+p_{w_{0}} F^{1}
\end{array}\right)
$$

and coefficients of $v_{2}$ give

$$
\Delta \cdot G_{v_{1}}-G \cdot \Delta_{v_{1}}=0 \text {, hence } G=g\left(x, u_{0}, v_{0}, w_{0}\right) \Delta
$$

for appropriate function $g$; however trivially $g=a_{0}$. With this result, we obtain

$$
\begin{aligned}
& \Delta\left(g_{u_{1}} \Delta+g \Delta_{u_{1}}\right)-g \Delta \cdot \Delta_{u_{1}} \\
& \quad=\Delta \operatorname{det}\left(\begin{array}{cc}
F_{1}^{1} & q_{v_{1}} F_{11} \\
F^{11} & q_{v_{1}} F_{1}^{1}
\end{array}\right)=(\Delta)^{2} q_{v_{1}}
\end{aligned}
$$

by inspection of coefficients of $v_{2}$. It follows that $g_{u_{1}}=q_{v_{1}}$, whence

$$
\begin{aligned}
& g=R\left(x, u_{0}, v_{0}, w_{0}\right) u_{1}+S\left(x, u_{0}, v_{0}, w_{0}\right), \\
& q=R\left(x, u_{0}, v_{0}, w_{0}\right) v_{1}+T\left(x, u_{0}, v_{0}, w_{0}\right) .
\end{aligned}
$$

Analogously the lower-order terms of the first equation (274) give

$$
\Delta \cdot \mathscr{D} q=\operatorname{det}\left(\begin{array}{cc}
p_{u_{0}}+p_{w_{0}} F_{1} & F_{11} \\
p_{v_{0}}+p_{w_{0}} F^{1} & F_{1}^{1}
\end{array}\right),
$$

while the second-order terms do not provide any new requirements.

Let us finally recall (275) with $a_{0}=g$ and $q$ given by (284) inserted. These equations turn into the compatible system

$$
\begin{aligned}
& p_{u_{1}}+\left(R u_{1}+S\right) F_{11}+\left(R v_{1}+T\right) F_{1}^{1}=0, \\
& p_{v_{1}}+\left(R u_{1}+S\right) F_{1}^{1}+\left(R v_{1}+T\right) F^{11}=0
\end{aligned}
$$

for the function $p$ with the solution

$$
\begin{array}{r}
p=\left(F-F_{1} u_{1}-F^{1} v_{1}\right) R-F_{1} S-F^{1} T+C \\
\left(C=C\left(x, u_{0}, v_{0}, w_{0}\right)\right) .
\end{array}
$$

Then (278) is expressed by the crucial requirement

$$
\left(F-F_{1} u_{1}-F^{1} v_{1}\right) \cdot \mathscr{D R}-F_{1} \cdot \mathscr{D S}-F^{1} \cdot \mathscr{D} T+\mathscr{D C}=0
$$

for the functions $R, S, T$ and $C$. One can moreover verify with the help of

$$
\begin{aligned}
& 0=(\mathscr{D} p)_{u_{1}}=\mathscr{D}\left(p_{u_{1}}\right)+p_{u_{0}}+p_{w_{0}} F_{1}, \\
& 0=(\mathscr{D} p)_{v_{1}}=\mathscr{D}\left(p_{v_{1}}\right)+p_{v_{0}}+p_{w_{0}} F^{1}
\end{aligned}
$$

that the remaining equations (281) and (285) become identities.

Let us summarize our achievements. Assuming $\left(F_{1}^{1}\right)^{2} \neq$ $F_{11} F^{11}$, all infinitesimal symmetries (268) are determined by formula (287), the second equation (284) and $z=$ $-q_{v_{1}}=-R$ with functions $R, S, T, C$ of variables $x_{0}, u_{0}, v_{0}$, $w_{0}$ satisfying (288).

Traditional methods are sufficient to analyze thoroughly (288). Passing to more details, we have

$$
\begin{gathered}
\left(F-F_{1} u_{1}-F^{1} v_{1}\right)\left(u_{1} R_{u_{0}}+v_{1} R_{v_{0}}+F R_{w_{0}}\right) \\
-F_{1}\left(S_{x}+u_{1}\left(S_{u_{0}}+R_{x}\right)+v_{1} S_{v_{0}}+F S_{w_{0}}\right) \\
-F^{1}\left(T_{x}+u_{1} T_{u_{0}}+v_{1}\left(T_{v_{0}}+R_{x}\right)+F T_{w_{0}}\right) \\
+C_{x}+u_{1} C_{u_{0}}+v_{1} C_{v_{0}}+F\left(C_{w_{0}}+R_{x}\right)=0 .
\end{gathered}
$$

Analogously as in Section 6, the large series of coefficients

$$
F u_{1}, F_{1}\left(u_{1}\right)^{2}, F^{1} v_{1} u_{1}, F v_{1}, F_{1} u_{1} v_{1}, \ldots, 1, u_{1}, v_{1}, F
$$

appears. If these functions are $\mathbb{R}$-linearly independent, only the solution $R, S, T, C$ such that

$$
\begin{aligned}
R_{u_{0}} & =R_{v_{0}}=R_{w_{0}}=S_{x}=S_{u_{0}}+R_{x} \\
& =\cdots=C_{v_{0}}=C_{w_{0}}+R_{x}=0
\end{aligned}
$$

is possible. It follows that

$$
\begin{array}{cc}
R=a_{1} x+a_{2}, & S=-a_{1} u_{0}+a_{3}, \\
T=-a_{1} v_{0}+a_{4}, & C=-a_{1} w_{0}+a_{5},
\end{array}
$$

where $a_{1}, \ldots, a_{5} \in \mathbb{R}$ are arbitrary constants. This result provides the obvious symmetries which are self-evident at a first glance, the coordinate shifts and the similarity.

For a special choice of function $F$, the symmetry group may be very large and less trivial. We can mention the case $F=u_{1} v_{1}$. Then the arising system of five equations

$$
\begin{aligned}
R_{x} & +S_{u_{0}}+T_{v_{0}}-C_{w_{0}} \\
& =R_{u_{0}}+T_{w_{0}} \\
& =R_{v_{0}}+S_{w_{0}}=T_{x}-C_{u_{0}}=S_{x}-C_{v_{0}}=0
\end{aligned}
$$

for the unknown functions

$$
\begin{gathered}
R=R\left(x, u_{0}, v_{0}\right), \quad S=S\left(x, u_{0}, w_{0}\right), \\
T=T\left(x, v_{0}, w_{0}\right)=C\left(u_{0}, v_{0}, w_{0}\right),
\end{gathered}
$$


can be resolved by

$$
\begin{gathered}
R=a_{1} x+a_{2} u_{0}+a_{3} v_{0}+a_{4}, \\
S=a_{5} x+a_{6} u_{0}-a_{3} w_{0}+a_{7}, \\
T=a_{8} x+a_{9} v_{0}-a_{2} w_{0}+a_{10}, \\
C=a_{8} u_{0}+a_{6} v_{0}+\left(a_{1}+a_{6}+a_{9}\right) w_{0}+a_{11},
\end{gathered}
$$

where $a_{1}, \ldots, a_{11} \in \mathbb{R}$ are arbitrary constants.

We omit more examples, in particular the interesting cases (with $\mathbb{R}$-linear dependence of functions $F-F_{1} u_{1}-$ $\left.F^{1} v_{1}, F_{1}, F^{1}, 1\right)$ where the infinitesimal symmetries depend on arbitrary functions and the "degenerate" cases when either $\Delta=0$ or $D F_{1}=D F^{1}=0$ identically.

(u) The Order-Increasing Infinitesimal Symmetry. Let us mention variations (268) satisfying moreover the equations

$$
\mathscr{L}_{Z} \pi_{0}^{1}=\mu_{0}^{1} \pi_{0}^{1}+\mu_{0}^{2} \pi_{0}^{2}+\mu_{1}^{2} \pi_{1}^{2}, \quad \mathscr{L}_{Z} \pi_{0}^{2}=\mu \pi_{0}^{2}
$$

which provide the order-increasing case, if $\mu_{1}^{2} \neq 0$. One can then obtain the resolving system

$$
\begin{array}{r}
z D F_{1}+F_{11} a_{1}+F_{1}^{1} D q-p_{u_{0}}-p_{w_{0}} F_{1} \\
=F_{11} a_{0}+F_{1}^{1} q+p_{u_{1}}=z+q_{v_{1}}=0
\end{array}
$$

for the unknown functions

$$
\begin{gathered}
p=p\left(x, u_{0}, v_{0}, w_{0}, u_{1}, v_{1}\right), \\
q=q\left(x, v_{0}, v_{1}\right), \quad z=z\left(x, v_{0}, v_{1}\right)
\end{gathered}
$$

and moreover formula

$$
\mu_{1}^{2}=F_{1}^{1} a_{0}+F^{11} q+p_{v_{1}}=-F_{1}^{1} \frac{q D F^{1}+D p}{D F_{1}}+F^{11} q+p_{v_{1}}
$$

for the coefficient $\mu_{1}^{2}$. We mention only the particular case $F=$ $u_{1} v_{1}$. Then the resolving system reads $-q_{v_{1}} v_{2}+D q-p_{u_{0}}-$ $p_{w_{0}} v_{1}=q+p_{u_{1}}=0$ and admits the solution

$$
p=-q u_{1}+\left(q_{x}+q_{v_{0}} v_{1}\right) u_{0}+P\left(x, v_{1} u_{0}-w_{0}, v_{0}, v_{1}\right) \text {, }
$$

where the functions $q=q\left(x, v_{0}, v_{1}\right)$ and $P=P\left(x, v_{1} u_{0}-\right.$ $\left.w_{0}, v_{0}, v_{1}\right)$ may be arbitrarily chosen. Since the above coefficient

$$
\mu_{1}^{2}=-\frac{1}{v_{2}}\left(q u_{2}+D p\right)+p_{v_{1}}
$$

does not in general vanish, we have a large family of orderincreasing infinitesimal symmetries.

(ui) Another Order-Increasing Case. Let us mention variations (268) satisfying the equations

$$
\mathscr{L}_{Z} \pi_{0}^{1}=\lambda^{1} \pi_{0}^{1}+\lambda^{2} \pi_{0}^{2}, \quad \mathscr{L}_{Z} \pi_{0}^{2}=\mu^{1} \pi_{0}^{1}+\mu^{2} \pi_{0}^{2}
$$

which provide an order-increasing case if $\lambda^{2} \neq 0$. The resolving system

$$
\begin{gathered}
z D F_{1}+F_{11} a_{1}+F_{1}^{1} D q-p_{u_{0}}-p_{w_{0}} F_{1}=0 \\
F_{11} a_{0}+F_{1}^{1} q+p_{u_{1}}=F_{1}^{1} a_{0}+F^{11} q+p_{v_{1}}=0 \\
q_{u_{0}}+q_{w_{0}} F_{1}=q_{v_{1}}+z=0
\end{gathered}
$$

for the unknown functions

$$
\begin{gathered}
p=p\left(x, u_{0}, v_{0}, w_{0}, u_{1}, v_{1}\right), \\
q=q\left(x, u_{0}, v_{0}, w_{0}, v_{1}\right), \\
z=z\left(x, u_{0}, v_{0}, w_{0}, v_{1}\right)
\end{gathered}
$$

looks more complicated. One can also obtain the formula

$$
\lambda^{2}=q_{v_{1}} D F^{1}-F_{1}^{1} a_{1}-F^{11} D q+p_{v_{0}}+p_{w_{0}} F^{1}
$$

for the important coefficient $\lambda^{2}$. Let us again mention only the particular case $F=u_{1} v_{1}$. Then the resolving system is simplified as

$$
\begin{aligned}
& q_{v_{1}} v_{2}-D q+p_{u_{0}}+p_{w_{0}} v_{1}=q+p_{u_{1}} \\
& \quad=q u_{2}+D p-p_{v_{1}} v_{2}=q_{u_{0}}+q_{w_{0}} v_{1}=0 .
\end{aligned}
$$

It follows immediately that $p=-q u_{1}+P$ and the resolving system is reduced to the equations

$$
\begin{gathered}
\mathscr{D} q=P_{u_{0}}+P_{w_{0}} v_{1}, \quad u_{1} \mathscr{D} q=\mathscr{D P}, \\
q_{u_{0}}+q_{w_{0}} v_{1}=0 \\
\left(\mathscr{D}=\frac{\partial}{\partial x}+u_{1} \frac{\partial}{\partial u_{0}}+v_{1} \frac{\partial}{\partial v_{0}}+F \frac{\partial}{\partial w_{0}}\right)
\end{gathered}
$$

for the unknown functions $P$ and $q$ of variables $x, u_{0}, v_{0}, w_{0}, v_{1}$. This implies that $q=\mathrm{Q}\left(x, w, v_{0}, v_{1}\right)$, where $w=w_{0}-v_{1} u_{0}$ and we obtain two equations

$$
Q_{x}+Q_{v_{0}} v_{1}=P_{u_{0}}+P_{w_{0}} v_{1}, \quad P_{x}+P_{v_{0}} v_{1}=0
$$

with the solution $P=\bar{P}\left(v_{0}-x v_{1}, w_{0}-u_{0} v_{1}, v_{1}\right)+\bar{Q}$, where $\bar{P}$ may be arbitrary function while $\bar{Q}=\bar{Q}\left(x, u_{0}, v_{0}, w_{0}, v_{1}\right)$ is a fixed particular solution of differential equation

$$
Q_{x}+Q_{v_{0}} v_{1}=\bar{Q}_{u_{0}}+\bar{Q}_{w_{0}} v_{1}
$$

satisfying moreover the identity $\bar{Q}_{x}+\bar{Q}_{v_{0}} v_{1}=0$. We may choose the particular solution $\bar{Q}=\left(Q_{x}+Q_{v_{0}} v_{1}\right) u_{0}$. Then the identity turns into the requirement $\left(\partial / \partial x+v_{1} \partial / \partial v_{0}\right)^{2} Q=0$ which is satisfied if

$$
Q=Q_{1}\left(w, v_{1}\right) x+Q_{0}\left(w, v_{1}\right) \quad\left(w=w_{0}-v_{1} u_{0}\right) .
$$

Altogether taken, we have obtained the final solution

$$
\begin{gathered}
p=-q u_{1}+\bar{P}+\left(Q_{x}+Q_{v_{0}} v_{1}\right) u_{0}, \\
q=Q=Q_{1} x+Q_{0},
\end{gathered}
$$


where

$$
\begin{gathered}
\bar{P}=\bar{P}\left(v_{0}-x v_{1}, w, v_{1}\right), \quad Q_{1}=Q_{1}\left(w, v_{1}\right), \\
Q_{0}=Q_{0}\left(w, v_{1}\right), \quad w=w_{0}-v_{1} u_{0}
\end{gathered}
$$

and $\bar{P}, Q_{1}, Q_{0}$ are quite arbitrary functions. The abovementioned coefficient $\lambda^{2}$ does not in general vanish. (Indeed, look at the top-order summands

$$
\lambda^{2}=\cdots-F_{1}^{1} a_{1}+\cdots=\cdots-a_{1}+\cdots,
$$

where $v_{2} a_{0}=\cdots+D p$ by virtue of (263); hence, $v_{2} a_{1}=\cdots+$ $D^{2} p=\cdots-D^{2}\left(q u_{1}\right)=\cdots-q u_{3}$ may be substituted.) We again have an order-increasing infinitesimal symmetry.

Remark 45. Variations $Z$ satisfying (269) preserve the Pfaffian system $\pi_{0}^{1}=\pi_{0}^{2}=\pi_{1}^{1}=0$ and therefore generate a group for analogous reasons as in Remark 29. Variations $Z$ satisfying (297) preserve the Pfaffian system $\pi_{0}^{1}=\pi_{0}^{2}=$ $\pi_{1}^{2}=0$ and the case of requirements (303) is quite trivial in this respect. It follows that we have indeed obtained the infinitesimal symmetries $Z$.

\section{Concluding Example on Order-Increasing Symmetries}

Passing from infinitesimal symmetries $Z$ to the true symmetries $\mathbf{m}$, the linear theory is replaced with highly nonlinear area of Pfaffian equations and the prolongation into involutiveness. In accordance with E. Cartan's notice, nobody should expect such easily available results as in the Lie's infinitesimal theory. Our modest aim is twofold: to perform an economical reduction of the symmetry problem to finite dimension and to point out a useful interrelation between appropriate variations $Z$ and one-parameter families $\mathbf{m}(t)$ of higher-order symmetries. We again deal only with (260).

( $\iota$ Setting the Problem. Let us deal with symmetries $\mathbf{m}$ such that

$$
\begin{aligned}
& \mathbf{m}^{*} \pi_{0}^{i} \\
& =a^{i} \alpha_{0}+b^{i} \beta_{0}+c^{i} \gamma_{0} \\
& =\left(a^{i}+F_{1} c^{i}\right) \alpha_{0}+\left(b^{i}+F^{1} c^{i}\right) \beta_{0}+c^{i} \pi_{0}^{1} \quad(i=1,2) .
\end{aligned}
$$

Invertibility of $\mathbf{m}$ is obviously ensured if

$$
\begin{gathered}
\operatorname{det}\left(\begin{array}{cc}
a^{1}+F_{1} c^{1} & a^{2}+F_{1} c^{2} \\
b^{1}+F^{1} c^{1} & b^{2}+F^{1} c^{2}
\end{array}\right)=0, \\
\operatorname{det}\left(\begin{array}{lll}
a^{1} & a^{2} & D a^{1}-u D a^{2} \\
b^{1} & b^{2} & D b^{1}-u D b^{2} \\
c^{1} & c^{2} & D c^{1}-u D c^{2}
\end{array}\right) \neq 0,
\end{gathered}
$$

where

$$
u=\frac{a^{1}+F_{1} c^{1}}{a^{2}+F_{1} c^{2}}=\frac{b^{1}+F_{1} c^{1}}{b^{2}+F_{1} c^{2}}
$$

We tacitly suppose $a^{2}+F_{1} c^{2} \neq 0, b^{2}+F^{1} c^{2} \neq 0$ and one can observe that the particular case $u=0$ provides the traditional order-preserving symmetries. Equations (315) can be simplified to the equivalent system of equations

$$
\begin{gathered}
\mathbf{m}^{*} \pi_{0}^{1}-u \mathbf{m}^{*} \pi_{0}^{2}=v \pi_{0}^{1}, \\
\mathbf{m}^{*} \pi_{0}^{2}=a \alpha_{0}+b \beta_{0}+c \gamma_{0},
\end{gathered}
$$

where $v=c^{1}-u c^{2}$ and $a=a^{2}, b=b^{2}, c=c^{2}$. The invertibility is ensured by the inequalities

$$
v \neq 0, \quad \operatorname{det}\left(\begin{array}{ccc}
a & F_{1} & D F_{1} \\
b & F^{1} & D F^{1} \\
c & -1 & 0
\end{array}\right) \neq 0 .
$$

Equations (318) will be represented by a Pfaffian system in a certain finite-dimensional space; however, let us again simplify the notation by bars; for example,

$$
\begin{gathered}
\bar{x}=\mathbf{m}^{*} x, \quad \bar{\pi}_{r}^{i}=\mathbf{m}^{*} \pi_{r}^{i}, \quad \bar{\alpha}_{r}=\mathbf{m}^{*} \alpha_{r}, \\
\bar{\beta}_{r}=\bar{\pi}_{r}^{2}=\mathbf{m}^{*} \beta_{r}=\mathbf{m}^{*} \pi_{r}^{2},
\end{gathered}
$$

and so like. Then we have the system

$$
\bar{\pi}_{0}^{1}-u \bar{\pi}_{0}^{2}=v \pi_{0}^{1}, \quad \bar{\pi}_{0}^{2}=a \alpha_{0}+b \beta_{0}+c \gamma_{0}
$$

which should be completed by the exterior derivatives

$$
\begin{aligned}
d \bar{\pi}_{0}^{1}-u d \bar{\pi}_{0}^{2}-d u \wedge \bar{\pi}_{0}^{2}=d v \wedge \pi_{0}^{1}+v d \pi_{0}^{1}, \\
d \bar{\pi}_{0}^{2}=d a \wedge \alpha_{0}+d b \wedge \beta_{0}+d c \wedge \gamma_{0} \\
\quad+d x \wedge\left(\left(a+F_{1} c\right) \alpha_{1}+\left(b+F^{1} c\right) \beta_{1}\right) .
\end{aligned}
$$

We refer to (264) for terms $d \pi_{0}^{1}, d \bar{\pi}_{0}^{1}$ appearing here. We have obtained the compatibility problem of (322). The familiar prolongation criterion can be shortly expressed as follows. All coefficients and variables with bars are functions of the primary jet variables. So we may suppose, for example,

$$
\begin{array}{r}
d u=U d x+\sum u_{r}^{1} \alpha_{r}+\sum u_{r}^{2} \beta_{r}+u_{0}^{3} \gamma_{0} \\
(U=D u)
\end{array}
$$

(with summands of uncertain lengths) and analogously for $d v, d a, d b, d c$ with a little adjustment for the differential

$$
\begin{array}{r}
d \bar{x}=\lambda\left(d x+\sum \lambda_{r}^{1} \alpha_{r}+\sum \lambda_{r}^{2} \beta_{r}+\lambda_{0}^{3} \gamma_{0}\right) \\
\left(\lambda=D \bar{x}=D \mathbf{m}^{*} x=D W\right) .
\end{array}
$$

Such substitutions into (322) should give identities. However, a short inspection of the summand $\bar{F}_{11} \bar{\alpha}_{0} \wedge \bar{\alpha}_{1}$ in differential $d \bar{\pi}_{0}^{1}$ implies that then necessarily either $u=0$ (the group case) or $F_{11}=0$ identically.

(u) A Particular Case. It follows that the assumption $F=$ $f\left(v_{1}\right) u_{1}+g\left(v_{1}\right)$ is necessary; however, let us again suppose 
$F=u_{1} v_{1}$ from now on. Then (321) may be retained and (322) become more explicit

$$
\begin{aligned}
d \bar{x} \wedge\left(\bar{\pi}_{1}^{1}-u \bar{\pi}_{1}^{2}\right)+\bar{\beta}_{0} \wedge \bar{\alpha}_{1}+\bar{\alpha}_{0} \wedge \bar{\beta}_{1}-d u \wedge \bar{\pi}_{0}^{2} \\
=d v \wedge \pi_{0}^{1}+v\left(d x \wedge \pi_{1}^{1}+\beta_{0} \wedge \alpha_{1}+\alpha_{0} \wedge \beta_{1}\right) \\
d \bar{x} \wedge \bar{\pi}_{1}^{2}=d a \wedge \alpha_{0}+d b \wedge \beta_{0}+d c \wedge \gamma_{0} \\
+d x \wedge\left(\left(a+v_{1} c\right) \alpha_{1}+\left(b+u_{1} c\right) \beta_{1}\right) .
\end{aligned}
$$

We turn to the prolongation procedure in more detail.

(ui) On the Equation (326). The prolongation should satisfy the identity

$$
\begin{aligned}
\lambda(d x & \left.+\sum \lambda_{r}^{1} \alpha_{r}+\sum \lambda_{r}^{2} \beta_{r}+\lambda_{0}^{3} \gamma_{0}\right) \\
& \wedge \frac{1}{\lambda}\left(A \alpha_{0}+B \beta_{0}+C \gamma_{0}+\left(a+v_{1} c\right) \alpha_{1}+\left(b+u_{1} c\right) \beta_{1}\right) \\
= & \left(A d x+\sum a_{r}^{1} \alpha_{r}+\sum a_{r}^{2} \beta_{r}+a_{0}^{3} \gamma_{0}\right) \wedge \alpha_{0} \\
& +\cdots+\left(C d x+\sum c_{r}^{1} \alpha_{r}+\sum c_{r}^{2} \beta_{r}+c_{0}^{3} \gamma_{0}\right) \wedge \gamma_{0} \\
& +d x \wedge\left(\left(a+v_{1} c\right) \alpha_{1}+\left(b+u_{1} c\right) \beta_{1}\right)
\end{aligned}
$$

where $A=D a, B=D b, C=D c$. All summands with factor $d x \wedge$ mutually cancel. Then we conclude that necessarily $\lambda_{r}^{1}=$ $\lambda_{r}^{2}=0(r>1)$ and also $a_{r}^{1}=\cdots=c_{r}^{2}=0(r>1)$. It follows that the problem is reduced to finite dimension: $\bar{x}, a, b, c$ are functions only of coordinates $x, u_{0}, v_{0}, u_{1}, v_{1}$. Even the explicit formulae can be easily obtained as follows

$$
\begin{gathered}
a_{1}^{1}=\lambda_{1}^{1} A-\lambda_{0}^{1}\left(a+v_{1} c\right), \ldots, b_{1}^{2}=\lambda_{1}^{2} B-\lambda_{0}^{2}\left(b+u_{1} c\right), \\
b_{0}^{1}-a_{0}^{2}=\lambda_{0}^{1} B-\lambda_{0}^{2} A, \\
c_{0}^{1}-a_{0}^{3}=\lambda_{0}^{1} C-\lambda_{0}^{3} A, \\
c_{0}^{2}-b_{0}^{3}=\lambda_{0}^{2} C-\lambda_{0}^{3} B
\end{gathered}
$$

for the prolongation where moreover $\lambda_{1}^{1}\left(b+u_{1} c\right)=\lambda_{1}^{2}\left(a+v_{1} c\right)$ is supposed.

( $v$ ) On the Equation (325). Calculations modulo $d x$ are also sufficient here. The prolongation should satisfy

$$
\begin{aligned}
\left(\lambda_{0}^{1} \alpha_{0}+\lambda_{0}^{2} \beta_{0}+\lambda_{0}^{3} \gamma_{0}+\lambda_{1}^{1} \alpha_{1}+\lambda_{1}^{2} \beta_{1}\right) & \wedge\left(V \pi_{0}^{1}+v \pi_{1}^{1}+U \bar{\pi}_{0}^{2}\right) \\
& -\left(\sum u_{r}^{1} \alpha_{r}+\sum u_{r}^{2} \beta_{r}+u_{0}^{3} \gamma_{0}\right) \wedge \bar{\pi}_{0}^{2} \\
& -\left(\sum v_{r}^{1} \alpha_{r}+\sum v_{r}^{2} \beta_{r}+v_{0}^{3} \gamma_{0}\right) \wedge \pi_{0}^{1} \\
= & -\bar{\beta}_{0} \wedge \bar{\alpha}_{1}-\bar{\alpha}_{0} \wedge \bar{\beta}_{1}+v\left(\beta_{0} \wedge \alpha_{1}+\alpha_{0} \wedge \beta_{1}\right),
\end{aligned}
$$

where $U=D u, V=D v$. We have used the identity

$$
\bar{\pi}_{1}^{1}-u \bar{\pi}_{1}^{2}=\frac{1}{\lambda} \mathscr{L}_{D}\left(\bar{\pi}_{0}^{1}-u \bar{\pi}_{0}^{2}\right)+\frac{U}{\lambda} \bar{\pi}_{0}^{2}
$$

where $\bar{\pi}_{0}^{1}-u \bar{\pi}_{0}^{2}=v \pi_{0}^{1}$ is moreover substituted. In order to prove the existence of prolongation, the right-hand side terms should be made more explicit. We recall the identity (263) which gives

$$
\begin{gathered}
\pi_{1}^{1}+v_{2} \alpha_{0}+u_{2} \pi_{0}^{2}=0, \\
\pi_{2}^{1}+v_{2} \alpha_{0}+v_{1} \alpha_{1}+u_{3} \pi_{0}^{2}+u_{2} \pi_{1}^{2}=0
\end{gathered}
$$

and uniquely determines the forms $\bar{\alpha}_{0}$ and $\bar{\alpha}_{1}$ in terms of forms $\bar{\pi}_{1}^{1}, \bar{\pi}_{0}^{2}, \bar{\pi}_{2}^{1}, \bar{\pi}_{1}^{2}$ and therefore in terms of contact forms $\alpha_{r}, \beta_{r}$, and $\gamma_{0}$, if the rule (265) is applied to the primary equations (321). The result is that

$$
\begin{aligned}
& \bar{\beta}_{0} \wedge \bar{\alpha}_{1}=\bar{\pi}_{0}^{2} \wedge(\text { a certain sum of forms } \\
& \left.\qquad \alpha_{0}, \beta_{0}, \gamma_{0}, \alpha_{1}, \beta_{1}, \alpha_{2}, \beta_{2}\right) \\
& \bar{\alpha}_{0} \wedge \bar{\beta}_{1}=-\frac{1}{\bar{v}_{2}}\left(\bar{u}_{2} \bar{\pi}_{0}^{2}+\bar{\pi}_{1}^{1}\right) \wedge(\text { a certain sum of forms } \\
& \left.\alpha_{0}, \beta_{0}, \gamma_{0}, \alpha_{1}, \beta_{1}\right) .
\end{aligned}
$$

On the other hand, inequalities (266) imply that the factors

$$
V \pi_{0}^{1}+v \pi_{1}^{1}+U \bar{\pi}_{0}^{2}, \bar{\pi}_{0}^{2}, \pi_{1}^{1}
$$

on the left-hand side of (329) are linearly independent and we conclude that the prolongation can be realized. Moreover $u$ becomes a function of second-order coordinates while $x$ and $v$ are functions of first-order coordinates $x, u_{0}, v_{0}, w_{0}, u_{1}, v$ as before. The problem is again reduced to finite-dimension; however, we do not state explicit formula for the prolongation here.

Remark 46. In accordance with Lie's classical theory, the existence of infinitesimal symmetries $Z$ (Figure 5(a)) is equivalent to the existence of a one-parameter group $\mathbf{m}(\lambda)$ of symmetries (Figures 3(a) and 3(b)) due to the solvability of the Lie system ensured by Theorem 24. Alas, the "genuine" higher-order symmetries (Figure 3(c)) cannot be obtained in this way and they rest on the toilsome mechanisms of Pfaffian systems. We nevertheless propose a hopeful conjecture as follows. Every one-parameter family $\mathbf{m}(t)$ of symmetries ensures the existence of many variations $Z(t)$ depending on parameter $t$ (Figure 5(b)). We believe that the converse can be proved as well: one-parameter families of symmetries can be reconstructed from a "sufficiently large" supply of variations. Indeed, if $t$ is regarded as additional variable of the underlying space, then the family $Z(t)$ turns into a single vector field.

In any case, the existence of many variations is a necessary condition for the existence of "genuine" higher-order symmetries and the following point $(\nu)$ will be instructive in this respect.

(v) On the Variations. If a one-parameter family $\mathbf{m}(t)=\mathbf{m}$ (abbreviation) satisfies (318) then the corresponding family 


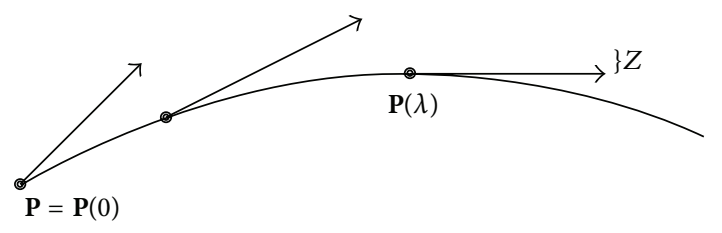

(a) Vector field $Z: Z_{\mathbf{P}(\lambda)}=(d / d \lambda) \mathbf{m}(\lambda) \mathbf{P}$

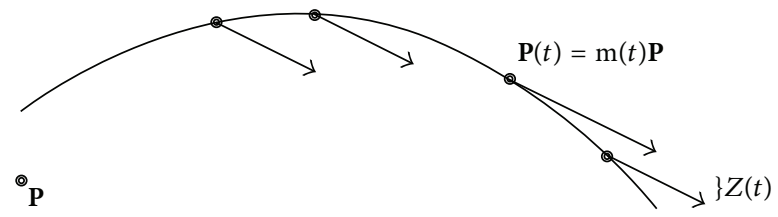

(b) Variations $Z(t): Z(t)_{\mathbf{P}(t)}=(d / d t) \mathbf{m}(t) \mathbf{P}$

Figure 5

$Z(t)=Z$ (abbreviation) of variations clearly satisfies the system

$$
\begin{gathered}
\mathscr{L}_{Z} \pi_{0}^{1}-u \mathscr{L}_{Z} \pi_{0}^{2}-u^{\prime} \pi_{0}^{2}=v^{\prime} \pi_{0}^{1}, \\
\mathscr{L}_{Z} \pi_{0}^{2}=a^{\prime} \alpha_{0}+b^{\prime} \beta_{0}+c^{\prime} \gamma_{0},
\end{gathered}
$$

where $u^{\prime}=Z u, \ldots, c^{\prime}=Z c$ may be regarded as new parameters. Assuming formula (268), one can obtain the resolving system

$$
\begin{aligned}
& z v_{2}+D q-p_{u_{0}}-a^{\prime} v_{1}+u q_{u_{0}} \\
& =q_{u_{0}}+a^{\prime} v_{1}+c^{\prime} v_{2}=0, \\
& z u_{2}+a_{1}-p_{v_{0}}-a^{\prime} u_{1}+u^{\prime}+u q_{v_{0}} \\
& =q_{v_{0}}+a^{\prime} u_{1}-b^{\prime}+c^{\prime} u_{2}=0, \\
& q+p_{u_{1}} \\
& =q_{u_{1}}=a_{0}+p_{v_{1}}=z+q_{v_{1}} \\
& =p_{w_{0}}-v^{\prime}-u q_{w_{0}}=q_{w_{0}}-a^{\prime}=0 .
\end{aligned}
$$

It follows from right-hand equations (337) that $p=-q u_{1}+\bar{q}$, where $q, \bar{q}$ do not depend on $u_{1}$. Recalling the identity

$$
a_{0} v_{2}+q u_{2}+D p=0
$$

then the middle equations (337) yield the conditions

$$
\begin{aligned}
q_{u_{0}}+q_{w_{0}} v_{1} & =\bar{q}_{x}+\bar{q}_{v_{0}} v_{1} \\
& =q_{x}+q_{v_{0}} v_{1}-\bar{q}_{u_{0}}-\bar{q}_{w_{0}} v_{1}=0
\end{aligned}
$$

and $q=q\left(x, u_{0}, v_{0}, v_{1}, w_{0}\right), \bar{q}=\bar{q}\left(x, u_{0}, v_{0}, v_{1}, w_{0}\right)$. With this result, (335) turns into identity and (336) reduces to the equation

$$
u^{\prime}=u\left(q_{w_{0}} u_{1}-q_{v_{0}}\right)+u_{2} q_{v_{1}}+D\left(q_{v_{1}} u_{1}-\bar{q}_{v_{1}}\right)+\bar{q}_{v_{0}}
$$

for the parameter $u^{\prime}$. Equations (339) are trivially satisfied if

$$
\begin{aligned}
& q=Q\left(x v_{1}-v_{0}, u_{0} v_{1}-w_{0}, v_{1}\right), \\
& \bar{q}=\bar{Q}\left(x v_{1}-v_{0}, u_{0} v_{1}-w_{0}, v_{1}\right) .
\end{aligned}
$$

There exist many variations corresponding to (318). The necessary condition for the existence of higher-order symmetries is satisfied.

Remark 47. Let us briefly sketch the connection to the general equivalence method [23] by using slightly adapted Cartan's notation. We consider space $\mathbb{R}^{n}$ (and its counterpart $\left.\overline{\mathbb{R}}^{n}\right)$ with coordinates $(x)=\left(x_{1}, \ldots, x_{n}\right)$ (or $(\bar{x})=\left(\bar{x}_{1}, \ldots, \bar{x}_{n}\right)$, resp.) and linearly independent 1 -forms $\omega_{1}, \ldots, \omega_{n}$ (and $\left.\bar{\omega}_{1}, \ldots, \bar{\omega}_{n}\right)$. In the classical equivalence problem, a mapping $\mathbf{m}$ should be determined such that

$$
\begin{aligned}
& \mathbf{m}^{*} \bar{\omega}_{i}=\sum a_{i j}(u) \omega_{j} \\
& \left(i, j=1, \ldots, n ;(x)=\mathbf{m}^{*}(\bar{x}), u=u(x)\right),
\end{aligned}
$$

where $\left(a_{i j}(u)\right)$ is a matrix of a linear group with parameters $(u)=\left(u_{1}, \ldots, u_{r}\right)$. In Cartan's approach, this requirement is made symmetrical:

$$
\begin{gathered}
\mathbf{m}^{*} \sum a_{i j}(\bar{u}) \bar{\omega}_{i}=\sum a_{i j}(u) \omega_{j} \\
\left((x)=\mathbf{m}^{*}(\bar{x}), \bar{u}=\bar{u}(x, u)\right) .
\end{gathered}
$$

This provides the invariant differential forms by appropriate simultaneous adjustments of both sides (343). Such procedure fails, if $\left(a_{i j}(x)\right)$ is not a matrix of a linear group which happens just in the case of higher-order symmetries on Figure 3(c). Then the corresponding total system (342) with $i, j=1,2, \ldots$ is invertible only in the infinite-dimensional underlying space $\mathbf{M}$ and $\left(a_{i j}(u)\right)$ need not be even a square matrix in any finite portion of the system (342). On the other hand, such a finite portion is quite sufficient since Lemma 17 ensures the extension on the total space $\mathbf{M}$. The "symmetrization" procedure cannot be applied, invariant differential forms need not exist, and only the common prolongation procedure is available, if the problem is reduced to a finite-dimensional subspace of $\mathbf{M}$.

\section{Concluding Survey}

Our approach to differential equations and our methods differ from the common traditional use. For better clarity, let us briefly report the main novelties as follows: clear interrelation between the external and internal concepts in Remarks 1 and 2; introduction and frequent use of "nonholonomic" series (18); the "absolute" and coordinate-free Definition 4 of ordinary differential equations; the distinction between variations and infinitesimal symmetries in Definition 8; the main tool, the standard bases generalizing the common contact forms in jet spaces; the invariance of constants $K=$ $K(\Omega)$ and $\mu=\mu(\Omega)$, the controllability concept related to the Mayer problem; the distinction between order-preserving, 
group-like, and true higher-order symmetries in Figure 1; technical Lemmas 17, 19, and 23 and Theorem 24 which provide new universal method of solution of the higherorder symmetry problem; new explicit formula as (136) for the famous and "well-known" symmetry problem of a Monge equation with two unknown functions; the Lagrange variational problem without Lagrange multipliers and with easy proofs; see Theorem 41; particular results of new kind for the Monge equation with three unknown functions; a note on the insufficience of $G$-structures in Remark 47.

All these achievements can be carried over the partial differential equations.

On this occasion, the actual extensive theory of the control systems

$$
\frac{d x}{d t}=f(x, u) \quad\left(t \in \mathbb{R}, x \in \mathbb{R}^{n}, u \in \mathbb{R}^{m}\right)
$$

is worth mentioning. It may be regarded as a mere formally adapted individual subcase of the theory of underdetermined systems of ordinary differential equations. However, the exceptional role of the independent variable $t$ (the change of notation), the state variables $x$, and the control $u$ is emphasized in applications; see [24-26] and references therein. In particular, only the $t$-preserving and moreover $t$-independent symmetries of the system (344) are accepted. So in our notation (1), such restriction means that we suppose $\bar{x}=$ $W=x$ and functions $W^{i}$ are independent of $x$. This is a fatal restriction of the impact of the theory of control systems. It follows that the results of this theory do not imply the classical results by Lie and Cartan; they are of rather special nature. The lack of new effective methods adapted to the control systems theory should be moreover noted. The absence of explicit solutions of particular examples is also symptomatic. Last but not least, unlike our diffieties, the control systems cannot be reasonably generalized for the partial differential equations.

We believe that the internal and higher-order approach to some nonholonomic theories are possible, for instance, in the case of the higher-order subriemannian geometry [12]. It seems that the advanced results [27] in the theory of geodesics can be appropriately adapted and rephrased in terms of invariants (as in [28]) instead of adjoint tensor fields.

\section{Appendix}

A nontrivial automorphism $\mathbf{m}$ of the jet space $\mathbf{M}(3)$ related to the theory of differential equation (260) is worth mentioning [9, pp. 44-46] without additional comments. In terms of usual jet coordinates $x, w_{r}^{i}(i=1,2,3 ; r=0,1, \ldots)$ on the space $\mathbf{M}(3)$, we put

$$
\mathbf{m}^{*} w_{0}^{1}=w_{1}^{1}, \quad \mathbf{m}^{*} w_{0}^{2}=w_{1}^{2}, \quad \mathbf{m}^{*} x=w_{1}^{3},
$$

and moreover

$$
\mathbf{m}^{*} w_{0}^{3}=\operatorname{det}\left(\begin{array}{ccc}
x w_{1}^{1}-w_{0}^{1} & \mathbf{m}^{*} w_{1}^{1} & \mathbf{m}^{*} w_{2}^{1} \\
x w_{1}^{2}-w_{0}^{2} & \mathbf{m}^{*} w_{1}^{2} & \mathbf{m}^{*} w_{2}^{2} \\
x w_{1}^{3}-w_{0}^{3} & 1 & 0
\end{array}\right) .
$$

The morphism $\mathbf{m}$ is rigorously defined since the transforms

$$
\mathbf{m}^{*} w_{1}^{j}=\frac{\mathbf{m}^{*} w_{0}^{j}}{\mathbf{m}^{*} x}, \quad \mathbf{m}^{*} w_{2}^{j}=\frac{\mathbf{m}^{*} w_{1}^{j}}{\mathbf{m}^{*} x} \quad(j=1,2)
$$

are well-known due to the prolongation (6). The point of construction is as follows. We have

$$
\begin{aligned}
\mathbf{m}^{*}\left(x-F\left(w_{0}^{1}, w_{0}^{2}\right)\right) & =\mathbf{m}^{*} x-F\left(\mathbf{m}^{*} w_{0}^{1}, \mathbf{m}^{*} w_{0}^{2}\right) \\
& =w_{1}^{3}-F\left(w_{1}^{1}, w_{1}^{2}\right) .
\end{aligned}
$$

So, assuming the invertibility of $\mathbf{m}$, differential equations (260) are identified with subspaces $\mathbf{N} \subset \mathbf{M}(3)$ given by equations $x=F\left(w_{0}^{1}, w_{0}^{2}\right)$. Every such a subspace $\mathbf{N}$ with given $F \neq$ const. is clearly isomorphic to the jet space $\mathbf{M}(2)$. We conclude that the diffiety $\Omega$ corresponding to given equation (260) is isomorphic to the diffiety $\Omega(2)$ of all curves in threedimensional space $\mathbb{R}^{3}$ and therefore admits huge supply of higher-order symmetries; see [4, Section 7] for quite simple examples.

Let us turn to the invertibility problem. We introduce a morphism $\mathbf{n}$ which will be identified with the sought inverse $\mathbf{m}^{-1}$. The definition is as follows. Let us introduce functions $a, b, c$ determined by three linear equations

$$
\begin{gathered}
\operatorname{det}\left(\begin{array}{ccc}
a & w_{1}^{1} & w_{2}^{1} \\
b & w_{1}^{2} & w_{2}^{2} \\
c & 1 & 0
\end{array}\right)=w_{0}^{3}, \\
\operatorname{det}\left(\begin{array}{ccc}
a & w_{1}^{1} & w_{3}^{1} \\
b & w_{1}^{2} & w_{3}^{2} \\
c & 1 & 0
\end{array}\right)=w_{1}^{3}, \\
\operatorname{det}\left(\begin{array}{ccc}
a & w_{2}^{1} & w_{3}^{1} \\
b & w_{2}^{2} & w_{3}^{2} \\
c & 0 & 0
\end{array}\right)+\operatorname{det}\left(\begin{array}{ccc}
a & w_{1}^{1} & w_{4}^{1} \\
b & w_{1}^{2} & w_{4}^{2} \\
c & 1 & 0
\end{array}\right)=w_{2}^{3} .
\end{gathered}
$$

It follows that functions $a, b, c$ moreover satisfy

$$
\operatorname{det}\left(\begin{array}{ccc}
D a & w_{1}^{1} & w_{2}^{1} \\
D b & w_{1}^{2} & w_{2}^{2} \\
D c & 1 & 0
\end{array}\right)=\operatorname{det}\left(\begin{array}{ccc}
D a & w_{1}^{1} & w_{3}^{1} \\
D b & w_{1}^{2} & w_{3}^{2} \\
D c & 1 & 0
\end{array}\right)=0
$$

whence the equations

$$
\begin{gathered}
D a=w_{1}^{1} \mathbf{n}^{*} x, \quad D b=w_{1}^{2} \mathbf{n}^{*} x, \\
D c=1 \cdot \mathbf{n}^{*} x=\mathbf{n}^{*} x
\end{gathered}
$$

uniquely define function $\mathbf{n}^{*} x$. We finally put

$$
\begin{gathered}
\mathbf{n}^{*} w_{0}^{1}=w_{0}^{1} \mathbf{n}^{*} x-a, \quad \mathbf{n}^{*} w_{0}^{2}=w_{0}^{2} \mathbf{n}^{*} x-b, \\
\mathbf{n}^{*} w_{0}^{3}=x \mathbf{n}^{*} x-c
\end{gathered}
$$

and then

$$
\begin{gathered}
D \mathbf{n}^{*} w_{0}^{1}=w_{1}^{1} \mathbf{n}^{*} x+w_{0}^{1} D \mathbf{n}^{*} x-D a=w_{0}^{1} D \mathbf{n}^{*} x, \\
D \mathbf{n}^{*} w_{0}^{2}=\cdots=w_{0}^{2} D \mathbf{n}^{*} x, \\
D \mathbf{n}^{*} w_{0}^{3}=\cdots=x .
\end{gathered}
$$


It follows that functions $w_{0}^{1}, w_{0}^{2}, x$ and hence $a, b, c$ and even the coordinate $w_{0}^{3}$ can be expressed in terms of certain pullbacks $\mathbf{n}^{*}$. Therefore $\mathbf{n}$ is invertible and moreover $\mathbf{n}=\mathbf{m}^{-1}$. Indeed, the last three equations read

$$
\begin{aligned}
& w_{0}^{1}=\frac{1}{D \mathbf{n}^{*} x} D \mathbf{n}^{*} w_{0}^{1}=\mathbf{n}^{*} D w_{0}^{1}=\mathbf{n}^{*} w_{1}^{1}, \\
& w_{0}^{2}=\cdots=\mathbf{n}^{*} w_{1}^{2}, \quad x=\cdots=\mathbf{n}^{*} w_{1}^{3}
\end{aligned}
$$

in full accordance with the initial equations (A.1) and formula (A.2) follows from (A.8).

\section{Conflict of Interests}

The authors declare that there is no conflict of interests regarding the publication of this paper.

\section{Acknowledgments}

This paper was elaborated with the financial support of the European Union's “Operational Programme Research and Development for Innovations," no. CZ.1.05/2.1.00/03.0097, as an activity of the regional centre AdMaS "Advanced Materials, Structures and Technologies."

\section{References}

[1] E. Cartan, "Les systèmes de Pfaff, à cinq variables et les équations aux dérivées partielles du second ordre," Annales Scientifiques de l'École Normale Supérieure. Troisième Série, vol. 27, pp. 109-192, 1910.

[2] V. Tryhuk and V. Chrastinová, "Automorphisms of curves," Journal of Nonlinear Mathematical Physics, vol. 16, no. 3, pp. 259-281, 2009.

[3] V. Chrastinová and V. Tryhuk, "Automorphisms of submanifolds," Advances in Difference Equations, vol. 2010, Article ID 202731, 26 pages, 2010.

[4] V. Tryhuk and V. Chrastinová, "On the mapping of jet spaces," Journal of Nonlinear Mathematical Physics, vol. 17, no. 3, pp. 293310, 2010.

[5] V. Tryhuk, V. Chrastinová, and O. Dlouhý, "The Lie group in infinite dimension," Abstract and Applied Analysis, vol. 2011, Article ID 919538, 35 pages, 2011.

[6] P. J. Olver, Applications of Lie Groups to Differential Equations, vol. 107 of Graduate Texts in Mathematics, Springer, New York, NY, USA, 1986.

[7] N. Kamran, Selected Topics in the Geometrical Study of Differential Equations, vol. 96 of CBMS Regional Conference Series in Mathematics, American Mathematical Society, Providence, RI, USA, 2002.

[8] I. S. Krasil'shchik, V. V. Lychagin, and A. M. Vinogradov, "Geometry of jet spaces and nonlinear partial differential equations," in Advanced Studies in Contemporary Mathematics, p. 441, Gordon and Breach Science Publishers, New York, NY, USA, 1986.

[9] J. Chrastina, The Formal Theory of Differential Equations, vol. 6 of Folia Facultatis Scientiarium Naturalium Universitatis Masarykianae Brunensis. Mathematica, Masaryk University, Brno, Czech Republic, 1998.
[10] S. Lie and G. Scheffers, Geometrie der Berürungstransformationen, Leipzig, Teubner, 1896.

[11] É. Goursat, "Leçons sur l'intégration des équations aux dérivées partielles premier ordre," Paris 1891; Zweite Auflage, J. Hermann, Paris, France, 1920.

[12] R. Montgomery, A Tour of Subriemannian Geometries, vol. 91 of Mathematical Surveys and Monographs, American Mathematical Society, Providence, RI, USA, 2002.

[13] V. Chrastinová and V. Tryhuk, "On the Mayer problem-I. General principles," Mathematica Slovaca, vol. 52, no. 5, pp. 555570, 2002.

[14] E. Cartan, "Le calcul des variations et certaines familles de courbes," Bulletin de la Société Mathématique de France, vol. 39, pp. 29-52, 1911.

[15] E. Cartan, "Sur l'equivalence absolu de certains systémes d'equations différentielles at sur certaines familles de courbes," Bulletin de la Société Mathématique de France, vol. 42, pp. 12-48, 1914.

[16] R. B. Gardner, The Method of Equivalence and Its Applications, vol. 58 of CBMS-NSF Regional Conference Series in Applied Mathematics, Society for Industrial and Applied Mathematics (SIAM), Philadelphia, Pa, USA, 1989.

[17] E. Cartan, "Sur quelques quadratures dont l'élément différentiel contient des fonctions arbitraires," Bulletin de la Société Mathématique de France, vol. 29, pp. 118-130, 1901.

[18] J. Chrastina, "Examples from the calculus of variations-I. Nondegenerate problems," Mathematica Bohemica, vol. 125, no. 1, pp. 55-76, 2000.

[19] J. Chrastina, "Examples from the calculus of variations-II. A degenerate problem," Mathematica Bohemica, vol. 125, no. 2, pp. 187-197, 2000.

[20] J. Chrastina, "Examples from the calculus of variations-III. Legendre and Jacobi conditions," Mathematica Bohemica, vol. 126, no. 1, pp. 93-111, 2001.

[21] J. Chrastina, "Examples from the calculus of variations-IV. Concluding review," Mathematica Bohemica, vol. 126, no. 4, pp. 691-710, 2001.

[22] P. A. Griffiths, "Exterior differential systems and the calculus of variations," in Progress in Mathematics, vol. 25, p. 335, Birkhuser, Boston, Mass, USA, 1983.

[23] E. Cartan, "Les sous-groupes des groupes continus de transformations," Annales Scientifiques de l'École Normale Supérieure. Troisième Série, vol. 25, no. 3, pp. 57-194, 1908.

[24] J.-B. Pomet, "A differential geometric setting for dynamic equivalence and dynamic linearization," in Geometry in Nonlinear Control and Differential Inclusions, vol. 32, pp. 319-339, Banach Center Publication, 1995.

[25] J.-B. Pomet, "A necessary condition for dynamic equivalence," SIAM Journal on Control and Optimization, vol. 48, no. 2, pp. 925-940, 2009.

[26] M. W. Stackpole, "Dynamic equivalence of control systems via infinite prolongation," to appear in Asian Journal of Mathematics, http://arxiv.org/pdf/1106.5437.pdf.

[27] J. Mikeš, A. Vanžurová, and I. Hinterleitner, Geodesic Mappings and Some Generalizations, Palacký University Olomouc, Faculty of Science, Olomouc, Czech Republic, 2009.

[28] S. S. Chern, "The geometry of differential equation $y^{\prime \prime \prime}=F(x$, $\left.y, y^{\prime}, y^{\prime \prime}\right), "$ Bull Sci. Math, pp. 206-212, 1939. 


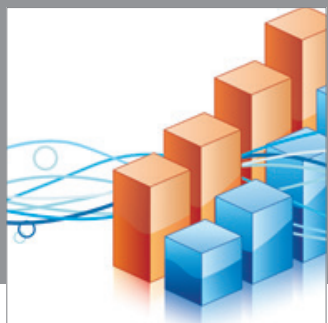

Advances in

Operations Research

mansans

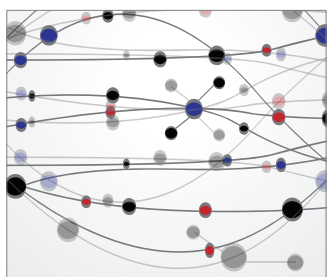

The Scientific World Journal
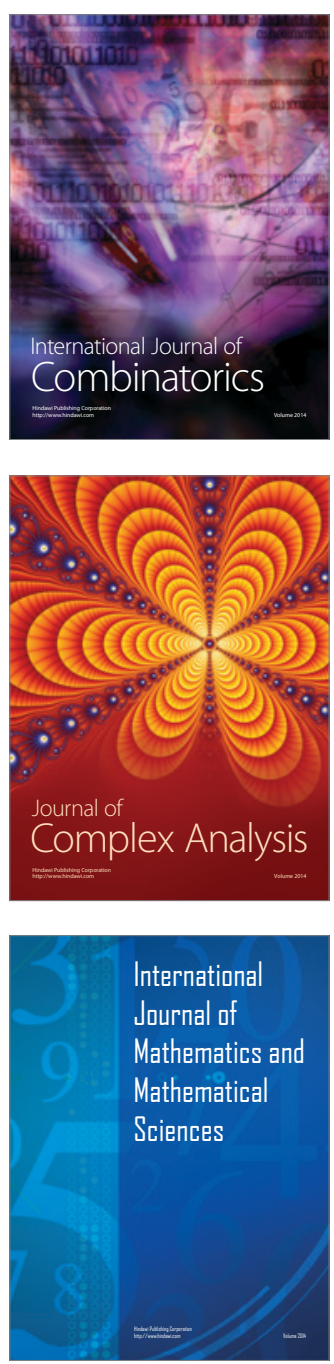
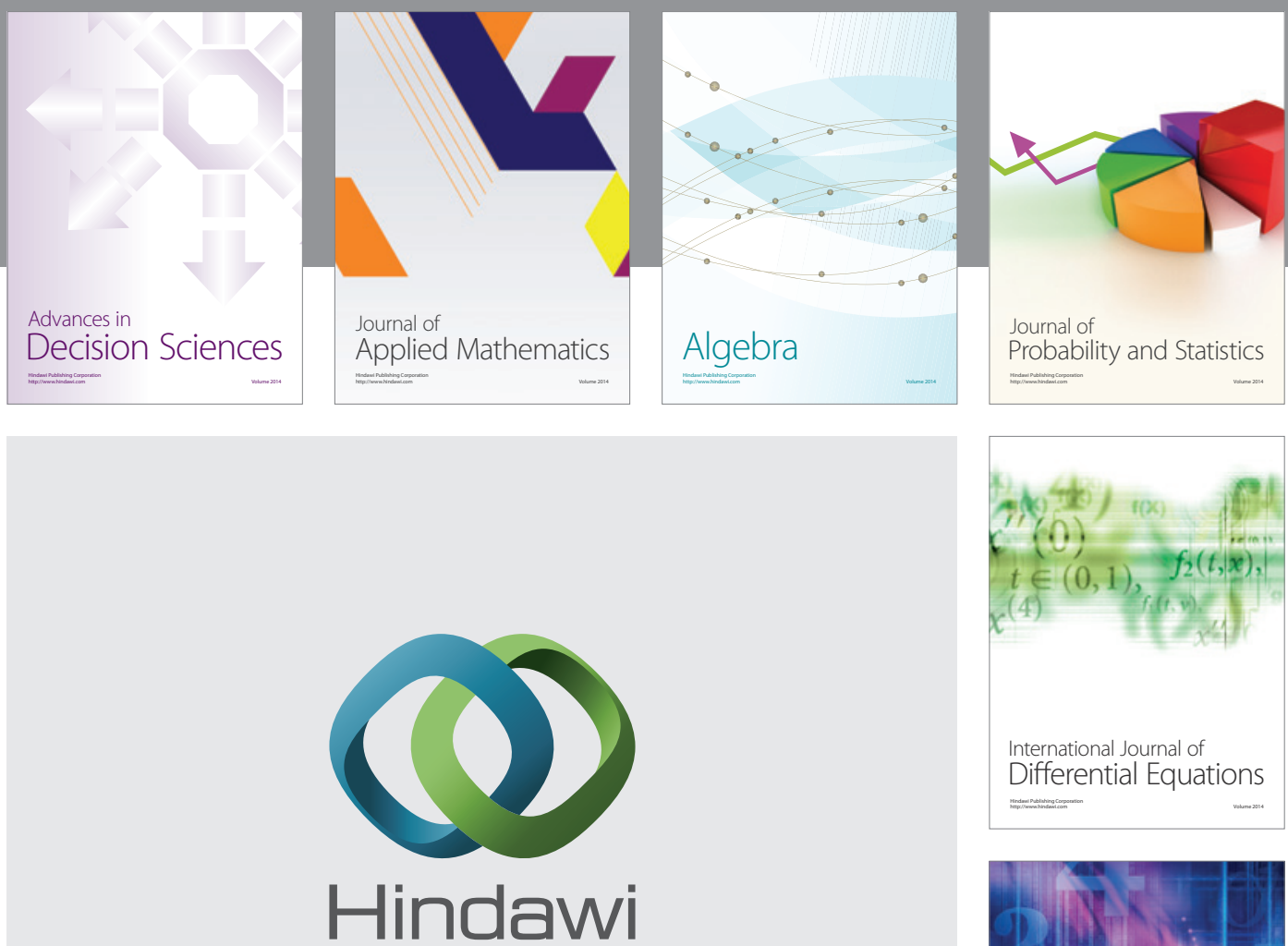

Submit your manuscripts at http://www.hindawi.com
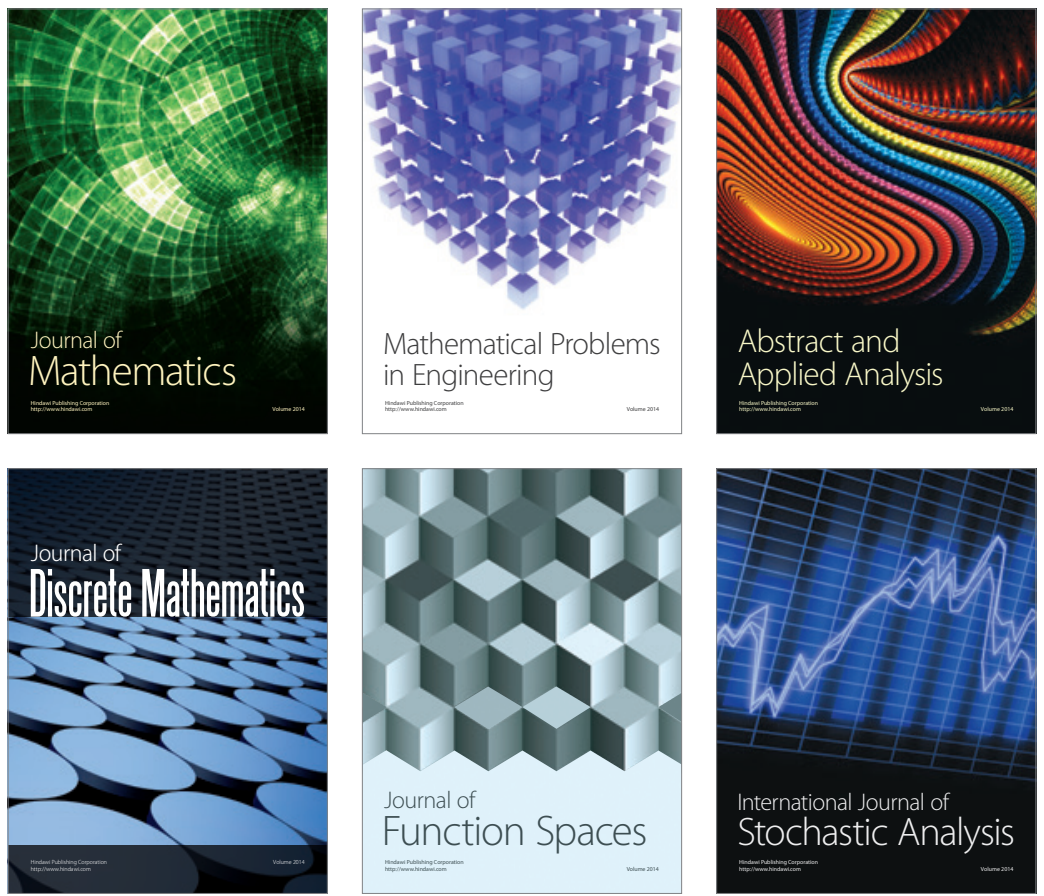

Journal of

Function Spaces

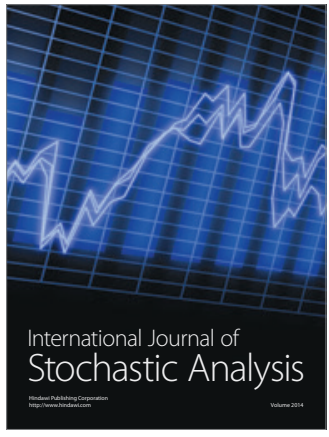

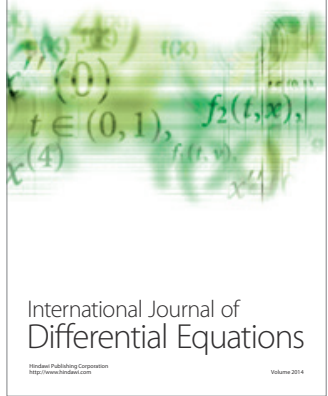
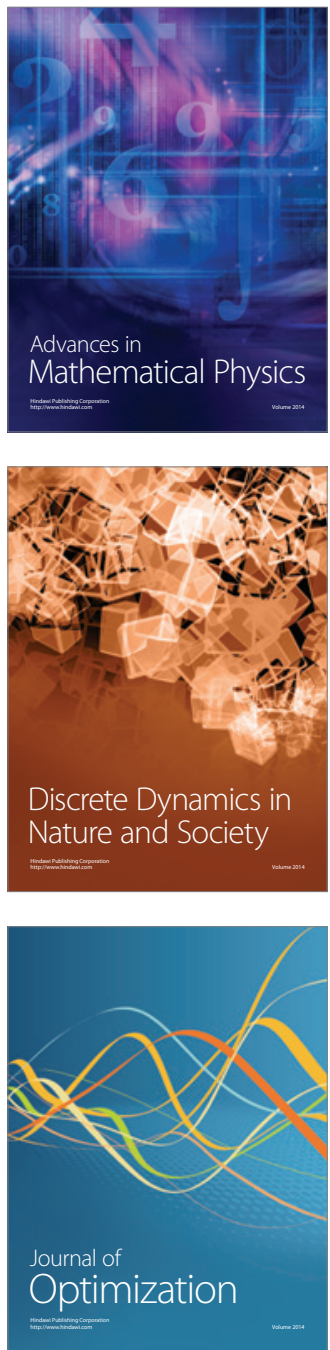